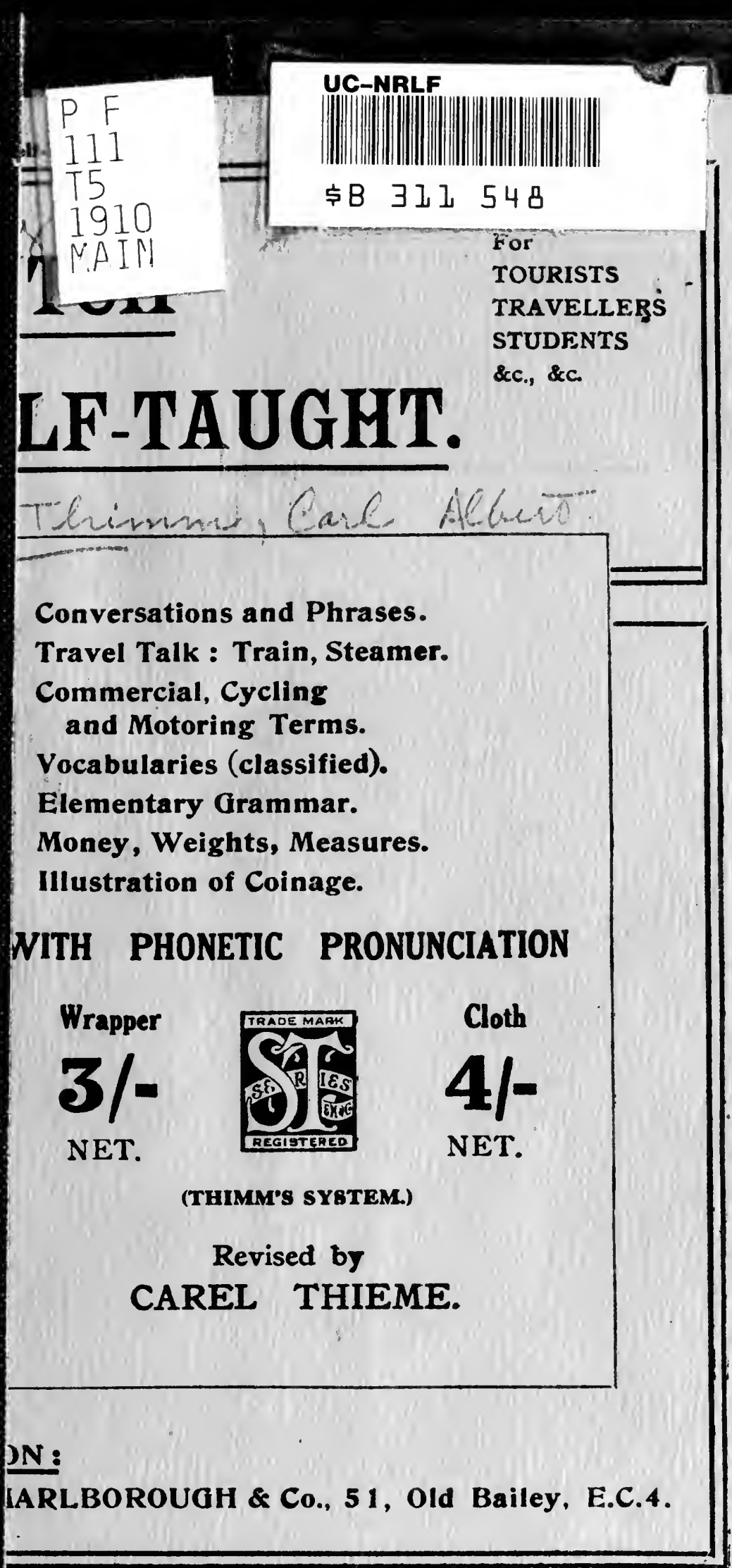




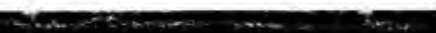


rough's Self-Taukht Series.

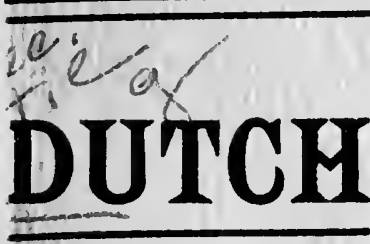

For

TOURISTS

TRAVELLERS

STUDENTS

\&c., \&c.

\section{SELF-TAUGHT.}

Conversations and Phrases.

Travel Talk : Train, Steamer.

Commercial, Cycling

and Motoring Terms.

Vocabularies (classified).

Elementary Grammar.

Money, Weights, Measures.

IIlustration of Coinage.

WITH PHONETIC PRONUNCIATION
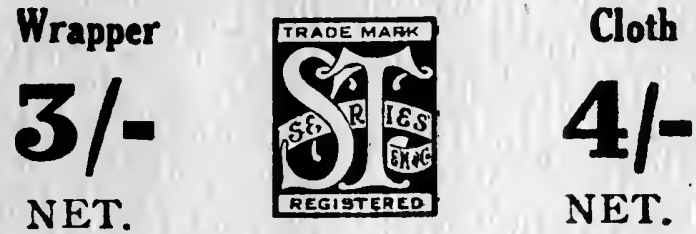

NET.

(THIMM'S SYBTEM.)

Revised by

CAREL THIEME.

\section{LONDON :}

E. MARLBOROUGH \& Co., 51 , Old Bailey, E.C.4. 


\section{GERMAN SELF-TAUGHT and GRAMMAR with KEY.}

By. W. E. WEBER. M.A., For Jearning the language by the Natural Method, with Pbonetic Pronunciation. Containing Notes on Articles, Gender, Accents, \&.c.; Classified Vocabularies; Conversational Phrases and Sentences; Travel Talk; Naval, Military and Commercial Terms; Tables of Money, \&.c., with Illustration of German Coinage. A course of simple and practical jessons in the Rules of Grammar exemplified with Model Sentences, Exerciscs and Reading Lessons in German Characters, Facsimile German Hand writing, Alphabetical Vocabulary, \&c.

In one volume. Crown 8vo. 332 pp. Green Cloth, 6i-net.

\section{GERMAN BY HOME STUDY.}

Contains German Self-Taught, Grammar and Key to the Exercises.

Banded together. Crown 8vo. 332 pp. Blue Wrapper 4/9 net

\section{GERMAN GRAMMAR SELF.TAUGHT.}

By II. E. IVEBER, II.A. Containing Niphabot and Pronunciation, Ru'es of Grammar evemplified with diod.I Sentences, Auxilar verbs, Irregular Verbs, List of the most common strong Verbs, Exercises and Reading Lessons in German Characters, Facsinile of Lerman Handwriting, Alphabetical Vocabulary.

Crown 8vo. 144 pp. Blue Wrapper, 2/- net. Red Cloth or Duxeen, 3'- net.

\section{KEY to German Grammar Self-Taught.}

Crown Svo. 40 pp. Blue Wrapper, 9d. net.

\section{GERMAN AND ENGLISH COMMERCIAL CORRESPONDENCE.}

(Marlhorough's Series of Englisi and Foreign Commercial Correspondence.)

By VON N. SADEZKY, and W. CHEVOB-MAURICE, A.I.L., London. Containing Model Phrases, Letters, Enquiries relating to Goods, Offers of Goods, Orders and their Execution, Shipping Correspondence, Agencies, Situations, Financial Credit, Insurance of Goods, Finance-llarkat Reports with Phrases, Debit and Credit, Arrangements and Failures, Announcements. Letters of Introduction. Complaints and Claims. Drafts, Inland and Foreign. Commercial Terms and Abbreviations-English-German, German-English. The written German Alphabet, the form of a Gernan Letter, \&c., \&c. Money, Weights and Measur?s, \&c., \&c., \&c.

Demy 8vo. Fawn wrapper, 2/- net.; Cloth, 3/-net.

\section{GERMAN LETTER-WRITER with KEY, Personal and Social.}

(Deutscher Briefsteller. In German and Roman Characters.)

By F. FRANCK. Revised and enlarged by J. C. H. SCHAFHAUSEN. ContainingINTronucrion: How a German letter should be written; the Form of a German Letter. Tirtes-Idiomatic Phrases and Polite Forms generally used at the beginning and end of Letters. LetTers-Notes, Orders, Commissions, Applications and Replies, Recommendation. Linquiries, \&.c.

Crown 8vo. $206 \mathrm{pp}$. Brown Cloth, 2/6 net.

\section{INTERIINEAR GERMIAN READING BOOK.}

By F. HAHN. With the literal English translation on the Hamiltonian System. Contaiaing a selection of tales by celebrated German Authors, progressively arranged either for use in classes, orfor Self-Study. Revised according to the new German Orthography Edited by C. A. THIMM.

$$
\text { Crown 8vo. } 101 \mathrm{pp} \text {. Wrapper, 1/. net. }
$$

\section{GERMAN TECHNICAL WORDS AND PHRASES.}

(Marltorough's Series of Foreign Technical Manuals. No. 2.)

An livglish-German and German-IENGlish Dictionary. By C. A. THIMII and W. Von KNOBI.ALCH. Containing Technic and Business Terms and Phrases used in Commerce, Arts, Sciences, Professions and Trades. With an Appendix of Tables of Money, Weights and Measures.

Second Edition. 16 mo. 241 pp. Cloth, red edges, $4 /$ - net 


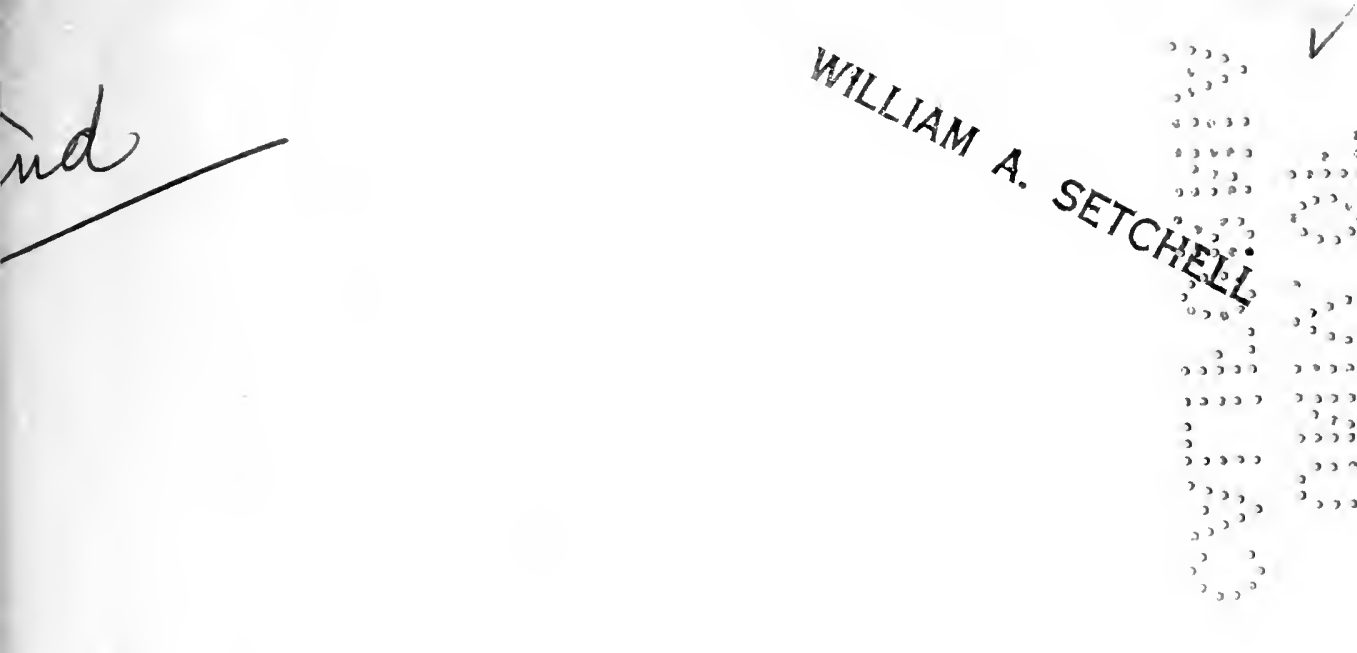




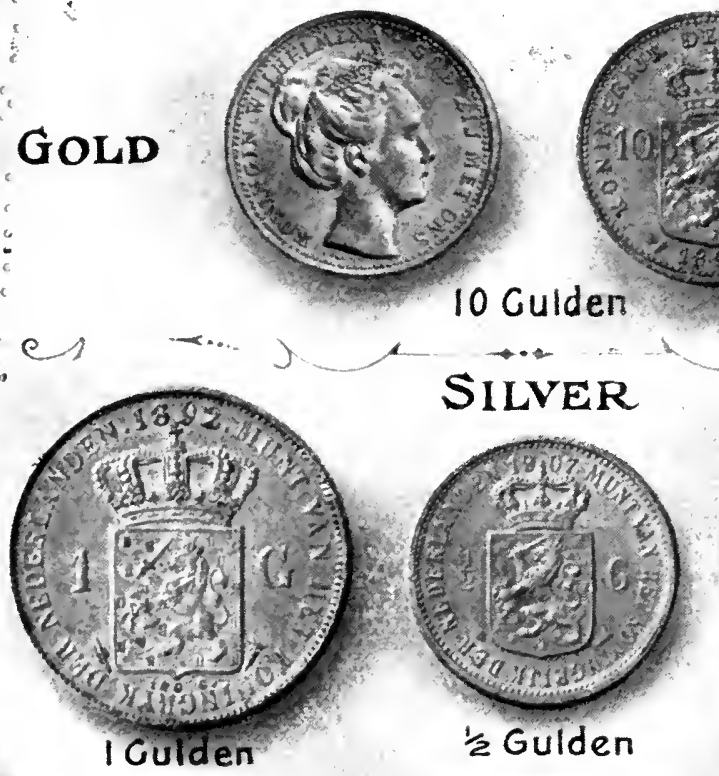


Dutch Self-Taught.

WITH

PHONETIC, PRONUNCIATION

B'

CAPTAIN C. A. THIMM.

Second Edition. Revised by

\section{CAREL THIEME,}

Examiner in Dutch for the London Chamber of Commerco

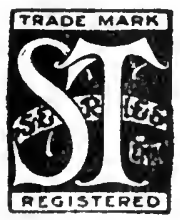

LONDON :

B. MaRlborough \& Co., 5I, Old Bailey, E.C. I9IO. 


\section{PREFACE.}

THE Dutch language being a collateral of the Modern German, there is considerable similarity between them, both having originated in the one form of construction, viz., old High German, and both having the same root words. This close relationship will naturally be found of great assistance to the student who, having a knowledge of either language, desires to learn the other.

The present manual is designed for students, and for those whose business or pleasure renders a practical acquaintance with the language a necessity, or at any rate a desideratum. Merchants, professional and commercial men visiting Holland or its dependencies, and travellers, officials, and settlers in South Africa will find it of great service and value. Dutch as spoken in Holland has been adhered to, for I found, whilst serving with the South African Field Force, that this is generally understood by the Boers and the Cape Dutch; moreover, it is this parent tongue that is being taught in the Government schools both in the Transvaal and the Orange River Colony. The very full outline of Grammar that forms a special and important feature of the work, will prove most helpful to the student and all who desire to master the language.

The phonetic or third column will be found to give, at a glance, the pronunciation of the Dutch words in the second column, as they are spoken by the educated classes in Holland. This is accomplished by means of Marlborough's System of Phonetics, as employed throughout their Series of "Self-Taught" Manuals. The system is so simple that those previously unacquainted with either Dutch or German can, with its aid, make themselves readily understood.

It is hoped that by facilitating the study and acquisition of the language, this Manual may help to strengthen the commercial and social ties between Great Britain and Holland, and also between the British and their Dutch fellow-subjects in South Africa.

In conclusion, I gladly acknowledge my indebtedness to Miss W. KLINkERT, of Amsterdam, for her valuable services in revising the proofs.

C. A. THIMM, Captain, late I.Y., South African Field Force.

London, 1904. 


\title{
DUTCH SELF-TAUGHT.
}

\section{The Alphabet with Phonetic Pronunciation.}

\section{The Dutch Alphabet has 26 letters:-}

Characters.

\author{
Name and English Pronanciation.
}

Phonetics

used.

A, a - $(a h)$ is either long or short.* When long, it is like $a$ in father, as laden [lah'de(r)n] $+\ldots$ ah When short, it has the same sound cut short, as brand [bra(h)nt] $\quad \ldots \quad \ldots . \quad \ldots$ a(h)

$\mathrm{B}, \mathrm{b}-($ bay) when final, is like $p$, as tob (top) $\quad \ldots \quad \mathrm{p}$ otherwise as in English

$\mathrm{C}, \mathrm{c}-($ say) before $a, o, u$, is like $k$, as canapé

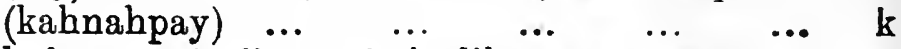
before $e, i, i j, z, e i$, is like $s$, as civiel (seeveel)

$\mathrm{Ch}, \mathrm{ch}-(\mathrm{ch})$ has no corresponding sound in English. It is a guttural aspirate resembling the ch in the Scotch word loch, but is much sharper, as macht. [ma(h)cht] $\quad$... $\quad . . \quad c h$

$\mathrm{D}, \mathrm{d}-($ day) at the end of a word is like $t$, as pad $[\mathrm{pa}(\mathrm{h}) \mathrm{t}]$

otherwise as in English

$\mathrm{E}, \boldsymbol{\theta}-(a y)$ when long is like ay in day, as zeven [zayfe(r)n]

when short, it is like $e$ in men, as vet (fet) Note. - At the end of a word, and in the prefixes be, ge, ver, and the suffixes $e l, e m$, $e n$, er, it is like $e$ in garden, as koude [kowde(r)], geladen [ge(r)lah'den] $\quad \ldots \quad$ e(r), $\theta$

F, f - (ef) as in English

G, g - (gay) has no corresponding sound in English ; it is a heavy guttural sound at the end of a word it is pronounced like ch (see above), as mag $[\mathrm{ma}(\mathrm{h}) c h] \quad \ldots \quad \ldots \quad c h$

* For rules relating to long and short vowels, see p. 10.

$+E(r)$ represents the sound of $e$ as in term; the (r) is added (where necessary) in the phonetics, merely in order to give the $e$ this sound-not to lengthen it-and must itself remain unheard. 
Characters.

Name and English Pronunciation.

Phonetics

$\mathrm{H}, \mathrm{h}-(\mathrm{hah})$ at the beginning of a word or syllable nsed. is always aspirated, as hagel (hah'gel)

After $t$ it is silent, as thans [ta(h)nss].

$\mathrm{I}, \mathrm{i}-(e e)$ when long, like ee in deep, as ivoor (ee'. vohr)

when short, like $i$ in pin, as wit (wit)...

$\mathrm{J}, \mathrm{j}-$ (yay) like $y$ in yet, as $j a$ (yah)

$\mathbf{K}, \mathbf{k}-(k a h)$ as in English, and always sounded before $n$, as knie (k-nee)

$\mathrm{L}, 1-(e l)$ as in English

$\mathbf{M}, \mathrm{m}-(\mathrm{em})$ as in English

$\mathrm{N}, \mathrm{n}-(e n)$ as in English

0 , o- (oh) when long, like $o$ in sole, as doren (doh'ren)

when short, like o in not, as los (loss)

ò

P, p - (pay) as in English

$\mathbf{Q}, \mathbf{q}-(k \hat{e u})$ like $k$, as bouquet. (boo'ket)

... $\quad$ ee

$\ldots$ i

$\cdots, \quad y$

y

k

$\begin{array}{ll}\cdots & \mathbf{k} \\ \cdots & \mathbf{l}\end{array}$

$\ldots \mathrm{m}$

.. $\mathrm{n}$

$\mathbf{R}, \mathbf{r}-(e r r)$ is pronounced with greater vibration than in English, and is never silent

$\mathbf{r}$

$\mathrm{S}, \mathrm{s}-(e s s)$ is a sharp sibilant, like $s s$ in loss, as mes (mess) ; never like $z$, as in lose

... s, ss

T, $\mathbf{t}-($ tay) as in English

$\mathrm{U}, \mathrm{u}-(\widehat{e u})$ is like the Frensh $u$, pronounced by forming the lips into a circle (as for whistling), and sounding " $\overparen{\mathrm{eu}}$ " as one sousd. Ex.: huren [hề'ren]

อิ

When between two consonants, it is like $u$ in mud, as juk (yuk)

u

$\mathbf{\nabla}, \boldsymbol{\nabla}-($ fay $)$ is like a soft $f$, as verdek $\left[\mathrm{fe}(\mathrm{r}) \mathrm{rdek}^{\prime}\right] \quad \ldots \quad \mathrm{f}$

$\mathrm{W}, \mathrm{w}-(v a y)$ is something like the English $w$, but the upper teeth should touch the lower lip in pronouncing it. Ex.: wijf (weyf) , ... w

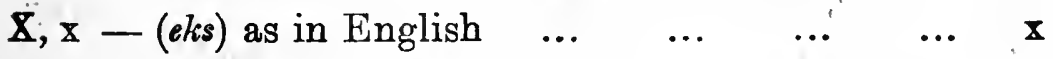

$\mathbf{Z} ; \mathrm{z}-(\mathrm{zed})$ as in English $\quad \ldots \quad \ldots \quad \ldots \quad \ldots \quad \ldots \quad$ z

NoTw.-C, Q, and X only occur in words borrowed from other languages. 


\section{Vowels, Diphthongs, Gutturals, \&c.}

The Vowels are employed as Simple Vowels, and also to form Diphthongs.

The Simple Vowels are Single and Double, viz., a, aa; $e$, $e e ;(-), e u ; i, i e ; o, o o ; u, u u$; also $(-), o e$.

For the Single Vowels see pp. 6 and 7 ; the Double Vowels are as follows:-

Phonetics used.

aa like $a$ in father, as raad (raht) $\quad \ldots \quad \ldots \quad \ldots a h$

ee $\quad$ "ay in day, as deel (dayl) $\quad \ldots \quad \ldots \quad \ldots \quad \ldots$ ay

ie like es in deep, as diep (deep) ... $\quad \ldots \quad \ldots \quad$ ee

oo $\quad$ "o in go, as vroom (frohm) $\quad \ldots \quad \ldots \quad$ oh

$u u \quad " u$ when long (see "Alphabet"), as $\begin{array}{llllll}\text { natuur (naht } \widehat{\mathrm{eu}} \text { ) } & \ldots & \ldots & \ldots & \ldots & \widehat{\mathrm{eu}}\end{array}$

eu something like $u$ in turn [not so open as $e(r)$, and longer]; better, like German $\ddot{o}$, as neus $\left[\begin{array}{lllllll}\mathrm{nu}(\mathrm{r}) \mathrm{ss}] & \ldots & \ldots & \ldots & \ldots & \mathrm{u}, \mathrm{u}(\mathrm{r})\end{array}\right.$

ob like oo in food, as boek (boo'k) $\quad \ldots \quad$...

The Diphthongs are $a u, o u, e i, i j, u i, a a i, e e u, o e i$, $o o i, i e u$, pronounced as follows :-

$\left.\begin{array}{l}a u \\ \text { ou }\end{array}\right\}$ like ow in cow, as $\left\{\begin{array}{lll}\begin{array}{l}\text { rauw (row)... } \\ \text { koud (kowt) }\end{array} & \ldots & \ldots\end{array}\right\}$ ow

$e i$ has no exact counterpart in English; it is somewhat like ey in they, very lightly pronounced, as meid (meyt) ... $\quad \ldots \quad \ldots$ ey

$i j \quad=e i$ (see above), as $\operatorname{dijk}$ (deyk) $\quad \ldots \quad \ldots \quad$ ey In the ending $l i j k(s), i j$ is like $e$ in term, as dagelijks [dahgele(r) $\mathrm{ks}] \quad \ldots \quad \ldots \quad \ldots e(\mathrm{r})$

ui like oy in toy, lightly and quickly passed over, as huis (hoyss) ... $\quad . . \quad \ldots \quad \ldots \quad \ldots$ oy

aai $=a a+i$ short, which see, as maaien [mah'i$\begin{array}{llllllll}\mathrm{e}(\mathrm{r}) \mathrm{n}] & \ldots & \ldots & \ldots & \ldots & \ldots & \ldots & \text { ah'i }\end{array}$

$o e i=o e+i$ short, which see, as moei (moo'i) $\quad \ldots \quad$ oo' $\mathrm{i}$

ooi $=o o+i$ short, which see, as hooi (hoh'i) $\quad \ldots$ oh'i 
Diphthongs (cont.) -

Phonetics

$e e u$ These diphthongs are always followed by $w$,

ien and the $u$ in each is silent, so that we get: :-

eeuw $=e e+w$, as leeuw $\left(\right.$ lay' $\left.^{\prime} \mathrm{w}\right) \quad \ldots \quad \ldots$ ay'w

ieuw $=i e+w$, as nieuw (nee'w) $\quad \ldots \quad \ldots e e^{\prime} w$

Note.-(1) When eeuw or ieuv is followed by another syllable, the $v$ is joined on to this syllable, as leeuwerik (lay'werik).

(2) Uw, as in zwaluw, $=u$ long $+w$; thus zwaluw is pronounced-zwa(h)leuw.

Gutturals, \&c.:-

$g$ and $c h$. These gutturals have the same sound at the end of a word, but must not be confounded, as they have different meanings ; e.g., ligt (licht), lies (verb); licht (licht),

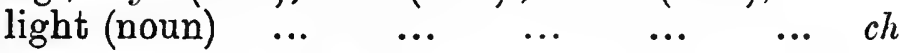

sch, at the beginning of a word, is pronounced

as $s$ and ch joined, as schip (schip) ... . ... sch

In the middle or at the end of a word, it is pronounced very like $s$, as tusschen [tus'se(r)n], bosch (boss) ‥ $\quad \ldots \quad \ldots$ s, ss

th is pronounced like $t$, as thans [ta(h)nss] $\quad \ldots \quad t$

$n g$ is a soft, one-consonant, nasal sound, pronounced like $n g$ in thing. It should never be divided into two sounds, as in finger, hunger, \&c.; e.g., vinger $\left[\mathrm{fing}^{\prime} \mathrm{e}(\mathrm{r}) \mathrm{r}\right]$... $\quad \ldots \quad$ ng

\section{PRELIMINARY NOTES.}

Pronunciation.-The student is recommended to familiarize himself at the outset with the few points in connection with the pronunciation of Dutch which present any difficulty, viz., the gutturals $\mathbf{g}$ and $\mathbf{c h}$, and the diphthongs $\mathbf{e i}, \mathbf{i j}, \mathbf{u i}$, and $\mathbf{e u}$. The phonetic signs $g$ and $c h$ convey no adequate idea of the gutturals, which should, if possible, be learned from a native of Holland. The same plan would be advantageous as regards $e i, i j$, ui, and $e u$, although their pronunciation is much simpler (see p. 8).

It is best to learn the Vocabularies and Conversations by heart, by repeating them aloud, following the pronunciation in the 
third column. Thus the tongue becomes expert in forming the words and the ear familiar with the sounds.

NoTk.-(1) Sometimes a consonant is doubled in the phonetics to keep the preceding vowel short, as blind (blinnt).

(2) $I g$ is pronouncd " $\mathrm{e}(\mathrm{r}) g$ (or $\mathrm{e}(\mathrm{r}) c h$ "), as in twintig [twinte(r)ch].

(3) Ir is like "ir" in " mirror," NOT as in "bird "; the phonetic sign nsed is ir (it\&lics), as firma (fir'mah).

Accent.-1. In words of more than one syllable the stress is laid mostly on the root syllable, as in prevelen [pray'fe(r)le(r)n].

2. Prefixes are accented, as in aartsbisschop (ahrts'bissop), antwoord [a(h)nt'wohrt], ondank [on'da(h)nk].

ExCEPTIONS: Be, ge, er, her, ont, ver; as in betalen [be(r)-tah'le(r)n], gelooven [ge(r)loh'fe(r)n].

3. Suffixes are Not accented; as in bedelaar [bay'de(r)lahr], lomperd [lom'pe(r)rt], kinderlijk [kin'de(r)rle(r)k], \&c.

ExCEPTIONS: Eel, egge, ei, es, $i j$, in, ier-which are accented; as in houweel (howayl'), prinses (prinsess'), \&c.

N.B.-The accented syllable is marked in the phonetics by the apostrophe ('), as above. In many Dutch words the accentuation follows the English or falls naturally, and the mark is omitted.

Long and Short Vowels.-I. DodBLE Vownes are always long, as paard (pahrt), stroom (strohm).

II. Sirgle Vowels are long or short, viz.:-

(a) Long at the end of a syllable, as mager [mah-ger], olifant [oh-lee-fa(h)nt], EXCEPT at the end of a word, as stille [stille(r)], kraai (krah'i), viooltje [fee-ohlt'ye(r)].

(b) Short-

(1) between two consonants in the same syllable, as redden. [red-de(r)n], kost (kosst), artisjok [a(h)r-tis-yok];

(2) in prefixes and suffixes, as gezang [ge(r)-za(h)ng], bezwijmen [be(r)-zweyme(r)n];

(3) before ch, as lucht (lucht), licht (licht).

Nots.-A single consonant commences a syllable, as eten [ay-te(r)n-not ayt-e(r)n], papegaai [pah-pe(r)-gah'i], zeeboezem [zay-boo-ze(r)m].

Where two consonants occur in the midale of a word, the syllables are divided between them, as vallen [fa(h) $\left.]^{\prime}-\mathrm{len}\right]$, donder [don:-de(r)r], EXCEPT when the preceding syllable is merely a PREFIX ending in a vowel, as geblaf [ge(r)-bla(h)f'].

Rules of Spelling.-1. The vowels a, e, o, $u$ are written single at the end of a syllable, when accented, as va'der, ste'den, lo'ven, mu'ren; EXCEPT in a number of words which have ee, and others which have oo, as hee'ten, koo'pen, \&o.

2. $I$ is written singie in the suffix isch, as godisch.

3. The double vowels ee, oo, le are written

(a) At the end of words of one syllable; as zee, stroo, drie.

(b) In suffixes; as hon weelen, Europeesche, werkelooze, massief, graniet.

4. Some words are written with either a single or double vowel, according to the meaning, as gene (that), geene (no); hopen (to hope), hoopen (beaps) ; kolen (coals), koolen (cabbages), \&o. 


\section{VOCABULARIES.}

1. The World and its Elements.

(De Wereld en hare Elementen.)

(See Notes on Gexder, pp. 69-71; ARTICLEs, p.69.)

\begin{tabular}{|c|c|c|}
\hline English. & Dutch. & \\
\hline $\begin{array}{l}\text { Air } \\
\text { cloud } \\
\text { cold } \\
\text { Creator } \\
\text { darkness } \\
\text { dew } \\
\text { dust } \\
\text { earth } \\
\text { East } \\
\text { eclipse } \\
\text { fire } \\
\text { fog } \\
\text { frost } \\
\text { God } \\
\text { hail } \\
\text { heat } \\
\text { Heaven } \\
\text { Hell } \\
\text { light } \\
\text { lightning } \\
\text { moon } \\
\text {-, full } \\
\text {-, new } \\
\text { moonlight } \\
\text { nature } \\
\text { North } \\
\text { rainbow } \\
\text { shade, shadow } \\
\text { sky } \\
\text { South } \\
\text { sun } \\
\text { thunder } \\
\text { weather } \\
\text { West } \\
\text { wind } \\
\text { world } \\
\end{array}$ & $\begin{array}{l}\text { lucht } \mathrm{f} . \\
\text { wolk } \mathrm{f} . \\
\text { koude } \mathrm{f} . \\
\text { Schepper } \mathrm{m} . \\
\text { duisternis } \mathrm{f} . \\
\text { dauw } \mathrm{m} . \\
\text { stof } \mathrm{n} . \\
\text { aarde } \mathrm{f} . \\
\text { oosten } \mathrm{n}, \\
\text { eclips } \mathrm{f} . \\
\text { vuur } \mathrm{n} ., \text { brand } \mathrm{m} . \\
\text { mist } \mathrm{m} . \\
\text { vorst } \mathrm{f} . \\
\text { God } \mathrm{m} . \\
\text { hagel } \mathrm{m} . \\
\text { hitte } \mathrm{f} . \\
\text { hemel } \mathrm{m} . \\
\text { hel } \mathrm{f} . \\
\text { licht } \mathrm{n} . \\
\text { bliksem } \mathrm{m} . \\
\text { maan } \mathrm{f} . \\
\text { volle maan } \mathrm{f} . \\
\text { nieuwe maan } \mathrm{f} . \\
\text { maanlicht } \mathrm{n} . \\
\text { natuur } \mathrm{f} . \\
\text { noorden } \mathrm{n} . \\
\text { regenboog } \mathrm{m} . \\
\text { schaduw } \mathrm{f} . \\
\text { lucht } \mathrm{f} . \\
\text { zuiden } \mathrm{n} . \\
\text { zon } \mathrm{f} . \\
\text { donder } \mathrm{m} . \\
\text { weder } \mathrm{n} . \\
\text { westen } \mathrm{n} . \\
\text { wind } \mathrm{m} . \\
\text { wereld } \mathrm{f} .\end{array}$ & $\begin{array}{l}\text { lucht } \\
\text { wol'k } \\
\text { kowde(r) } \\
\text { schep'pe(r)r } \\
\text { doyss'te(r)rniss } \\
\text { dow (like cow) } \\
\text { stoff } \\
\text { ahrde(r) } \\
\text { ohs'te(r)n } \\
\text { ayklipss' } \\
\text { feur, bra(h)nt } \\
\text { misst } \\
\text { forrst } \\
\text { got } \\
\text { hah'ge(r)l } \\
\text { hitte(r) } \\
\text { hay'me(r)l } \\
\text { hel } \\
\text { licht } \\
\text { blik'se(r)m } \\
\text { mahn } \\
\text { fol'le(r) mahn } \\
\text { nee'we(r)mahn } \\
\text { mahnlicht } \\
\text { nahteur' } \\
\text { nohr'de(r)n } \\
\text { ray'ge(r)n-bohch } \\
\text { scha(h)deuw } \\
\text { lucht } \\
\text { zoy'de(r)n } \\
\text { zon } \\
\text { don'de(r)r } \\
\text { way'de(r)r } \\
\text { wess'te(r)n } \\
\text { winnt } \\
\text { way're(r)lt }\end{array}$ \\
\hline
\end{tabular}


2. Land and Water. (Land en Water.)

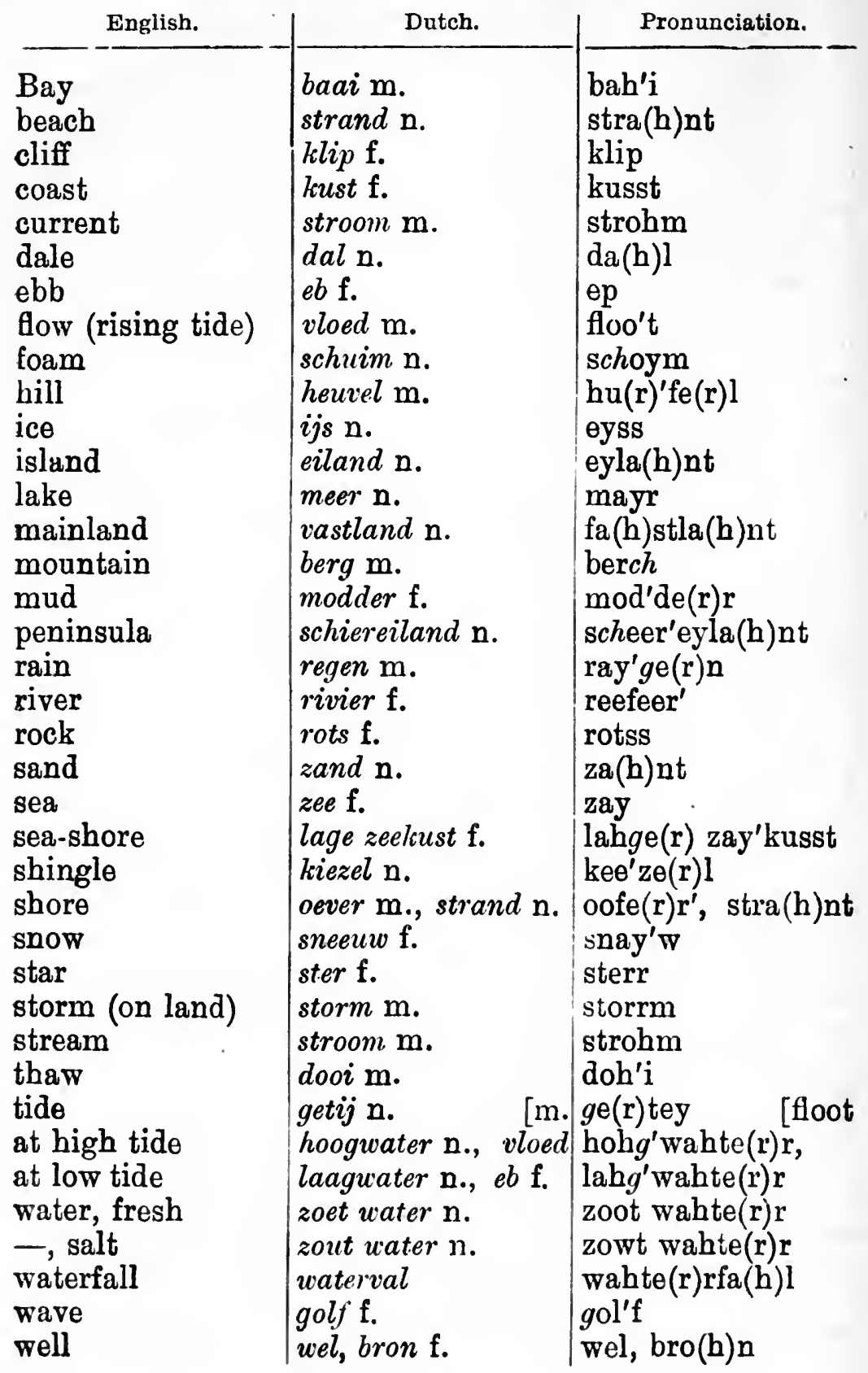


3. Minerals and Metals. (Mineralen en Metalen.)

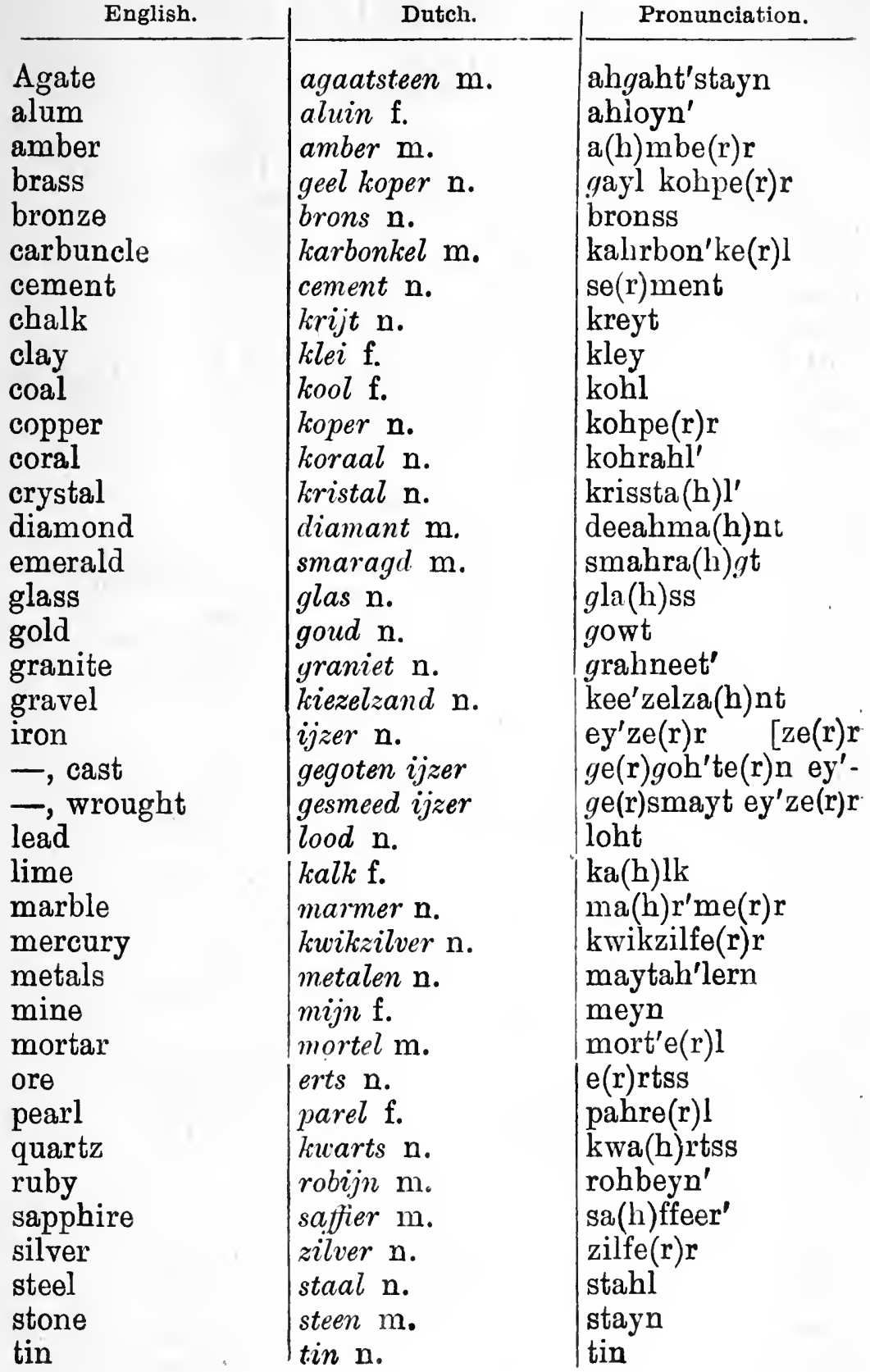


4. Animals, Birds, and Fishes.

(Dieren, Vogels en Visschen.)

\begin{tabular}{|c|c|c|}
\hline English. & Datch. & Pronunciation. \\
\hline $\begin{array}{l}\text { Animal } \\
\text { antelope } \\
\text { barking } \\
\text { bear } \\
\text { bird } \\
\text { blackbird } \\
\text { bleating } \\
\text { bull } \\
\text { calf } \\
\text { carp } \\
\text { cat } \\
\text { cattle } \\
\text { chicken } \\
\text { claw } \\
\text { cock } \\
\text { cod } \\
\text { colt } \\
\text { cow } \\
\text { crab } \\
\text { crow } \\
\text { cuckoo } \\
\text { dog } \\
\text { donkey } \\
\text { dove } \\
\text { duck } \\
\text { eagle } \\
\text { eel } \\
\text { elephant } \\
\text { feather } \\
\text { fins } \\
\text { fish } \\
\text { fox } \\
\text { fur } \\
\text { gills } \\
\text { goat } \\
\text { goose } \\
\text { gouse }\end{array}$ & 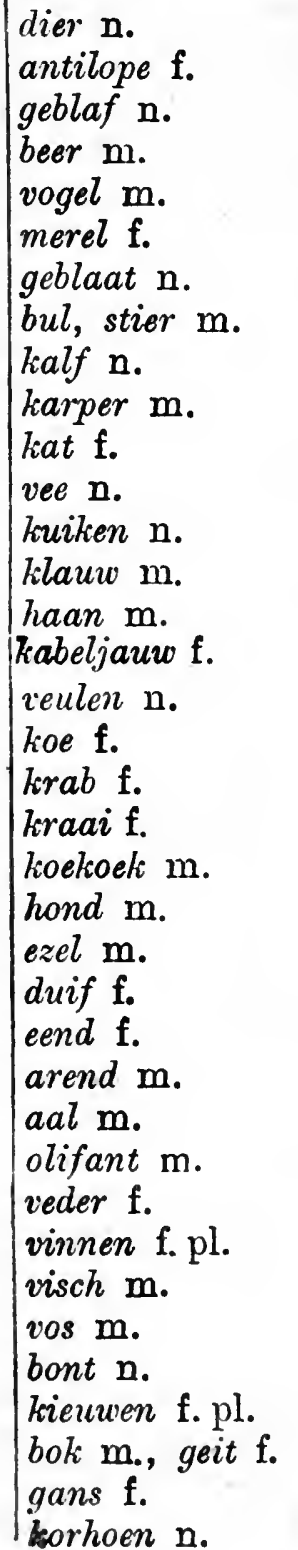 & $\begin{array}{l}\text { deer } \\
\text { a(h)nteelohpe(r) } \\
\text { ge(r)bla(h)f } \\
\text { bayr } \\
\text { foh'ge(r)l } \\
\text { mayre(r)l } \\
\text { ge(r)blaht' } \\
\text { bul (like but), steer } \\
\text { ka(h)lf } \\
\text { ka(h)rpe(r)r } \\
\text { ka(h)t } \\
\text { fay } \\
\text { koyke(r)n } \\
\text { klow (like cow) } \\
\text { hahn } \\
\text { ka(h)belyow } \\
\text { fu(r)'le(r)n } \\
\text { koo } \\
\text { kra(h)p } \\
\text { krah'i } \\
\text { koo'kook } \\
\text { hont } \\
\text { ay'ze(r)l } \\
\text { doyf } \\
\text { aynt } \\
\text { ahre(r)nt } \\
\text { ahl } \\
\text { oh'leefa(h)nt } \\
\text { fay'de(r)r } \\
\text { fin'ne(r)n } \\
\text { fiss } \\
\text { foss } \\
\text { bont } \\
\text { kee'we(r)n } \\
\text { bok, geyt } \\
\text { ga(b)nss } \\
\text { korr'hoon }\end{array}$ \\
\hline
\end{tabular}




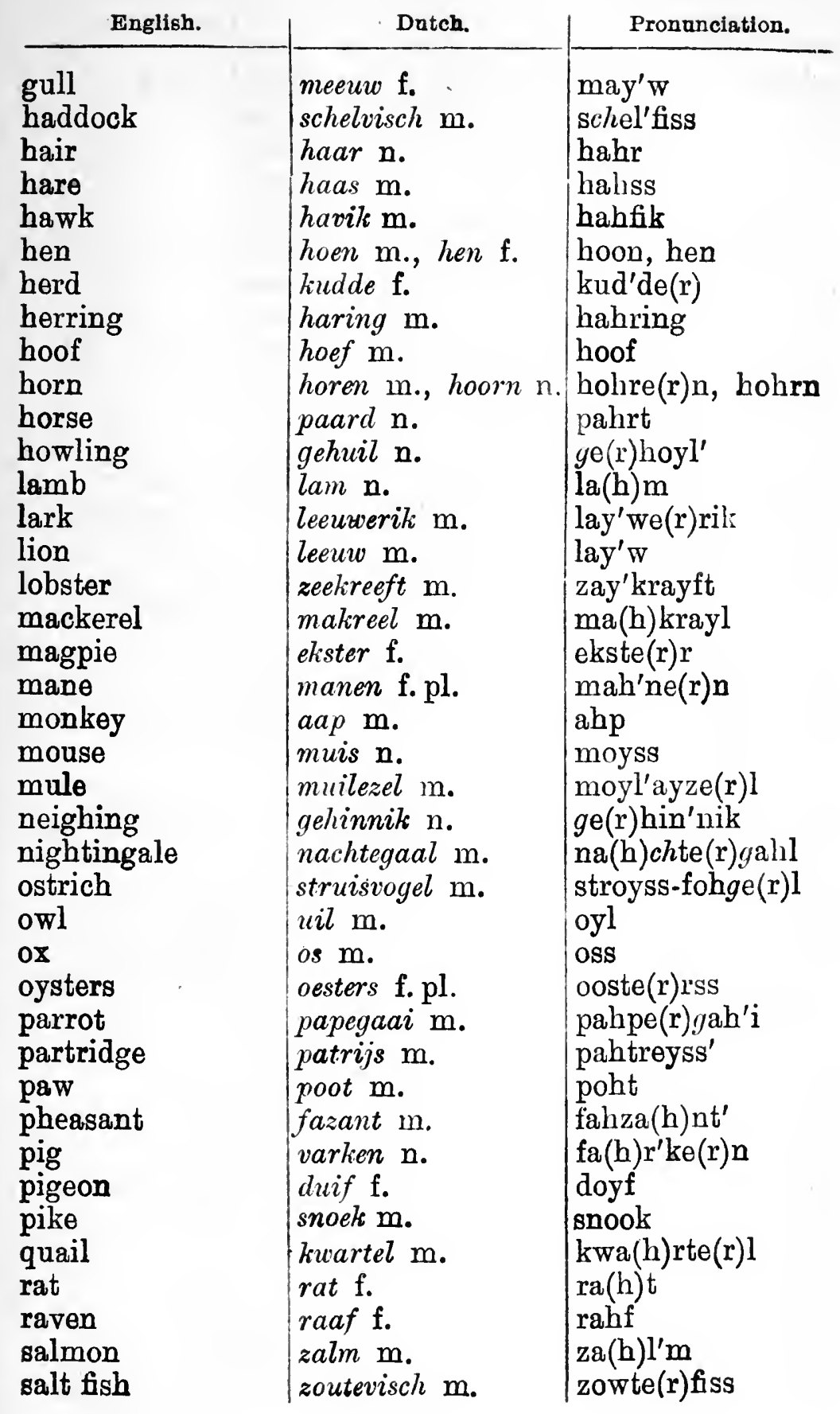




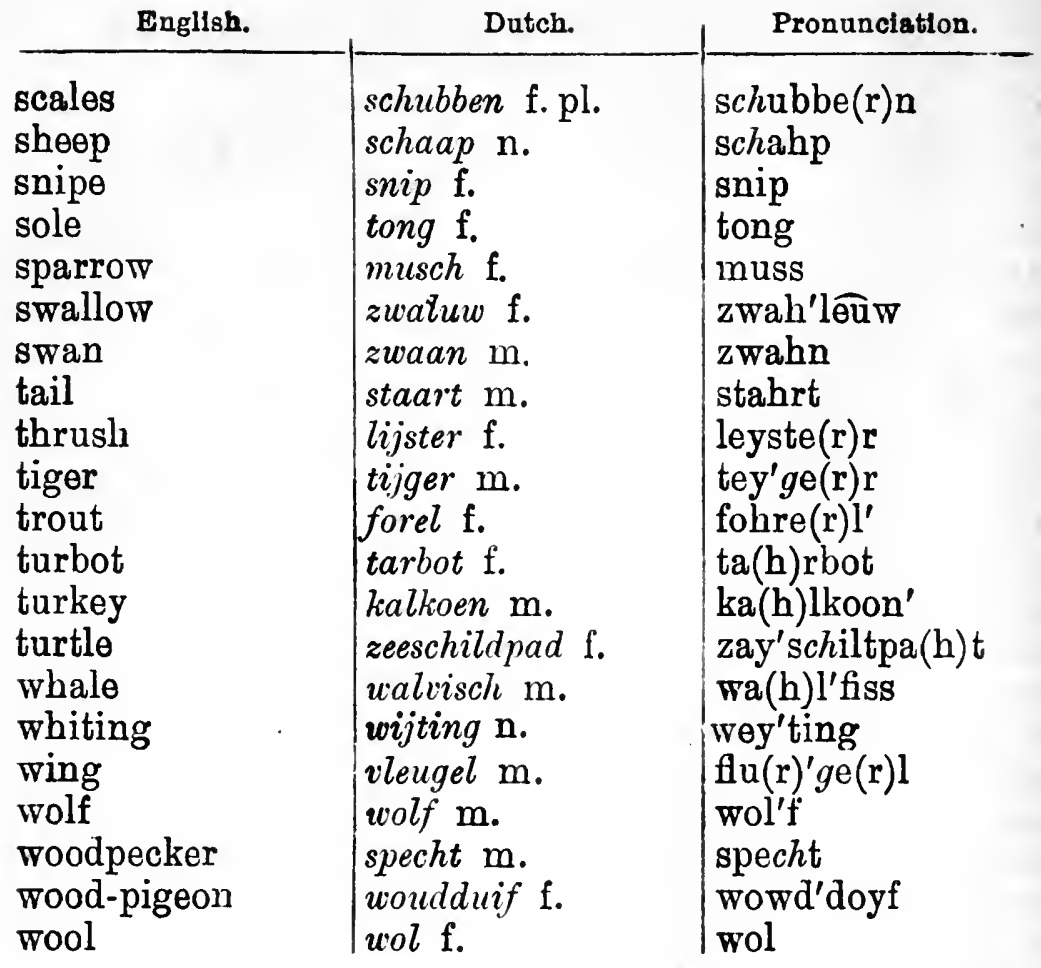

5. Reptiles \& Insects. (Kruipend Gedierte en Insecten.)

\begin{tabular}{|c|c|c|}
\hline $\begin{array}{l}\text { Ant } \\
\text { bee } \\
\text { beetle } \\
\text { butterfly } \\
\text { caterpillar } \\
\text { flea } \\
\text { fly } \\
\text { frog } \\
\text { gnat } \\
\text { grasshopper } \\
\text { mosquito } \\
\text { moth } \\
\text { serpent } \\
\text { silkworm } \\
\text { snail } \\
\text { snake }\end{array}$ & \begin{tabular}{|l|} 
mier f. \\
(honig) bij $\mathrm{f}$. \\
tor, kever m. \\
kapel f., vlinder $\mathrm{m}$. \\
rups f. \\
vloo $\mathrm{f}$. \\
vlieg $\mathrm{f}$. \\
kikvorsch $\mathrm{m}$. \\
mug f. \\
sprinkhaan m. \\
muskiet $\mathrm{m}$. \\
mot $\mathrm{f}$. \\
slang f. \\
zijworm $\mathrm{m}$. \\
slak f. \\
slang $\mathrm{f}$.
\end{tabular} & $\begin{array}{l}\text { meer } \\
\text { (hohning) bey } \\
\text { tor, kayfe(r)r } \\
\text { kahpell', flin'de(r)r } \\
\text { rups } \\
\text { floh } \\
\text { fleech } \\
\text { kikforss } \\
\text { much } \\
\text { sprink'hahn } \\
\text { muskeet' } \\
\text { mot } \\
\text { sla(h)ng } \\
\text { zeyworm } \\
\text { sla(h)k } \\
\text { sla(h)ng }\end{array}$ \\
\hline
\end{tabular}




\begin{tabular}{l|l|l}
\multicolumn{1}{c|}{ English. } & \multicolumn{1}{|c}{ Dutch. } & \multicolumn{1}{|c}{ Pronunciation. } \\
\hline spider & spin f. & spin \\
sting & angel $\mathrm{m}$. & $\mathrm{a}(\mathrm{h}) \mathrm{ng} \mathrm{g}^{\prime} \mathrm{e}(\mathrm{r}) \mathrm{l}$ \\
toad & pad f. & pa(h)t \\
viper & adder f. & $\mathrm{a}(\mathrm{h}) \mathrm{d}^{\prime} \mathrm{de}(\mathrm{r}) \mathrm{r}$ \\
wasp & wesp f. & wesp \\
worm & worm $\mathrm{m}$. & wor'm
\end{tabular}

6. Fruits, Trees, Flowers, and Vegetables. (Vruchten, Boomen, Bloemen en Groenten.)

(See Shopping, p. 109.)

\begin{tabular}{|c|c|c|}
\hline $\begin{array}{l}\text { Apple } \\
\text { artichoke } \\
\text { ash } \\
\text { asparagus } \\
\text { beans } \\
\text { beech-tree } \\
\text { beetroot } \\
\text { birch } \\
\text { blackberry } \\
\text { bouquet } \\
\text { branch } \\
\text { cabbage } \\
\text { carrot } \\
\text { cauliflower } \\
\text { celery } \\
\text { cherry } \\
\text { chestnut } \\
\text { cinnamon } \\
\text { crocus } \\
\text { cucumber } \\
\text { currant, black } \\
\text { daffodil } \\
\text { fagot } \\
\text { fig-tree } \\
\text { filbert } \\
\text { fir-tree } \\
\text { flower } \\
\text { forest } \\
\text { garlic } \\
\text { ginger }\end{array}$ & 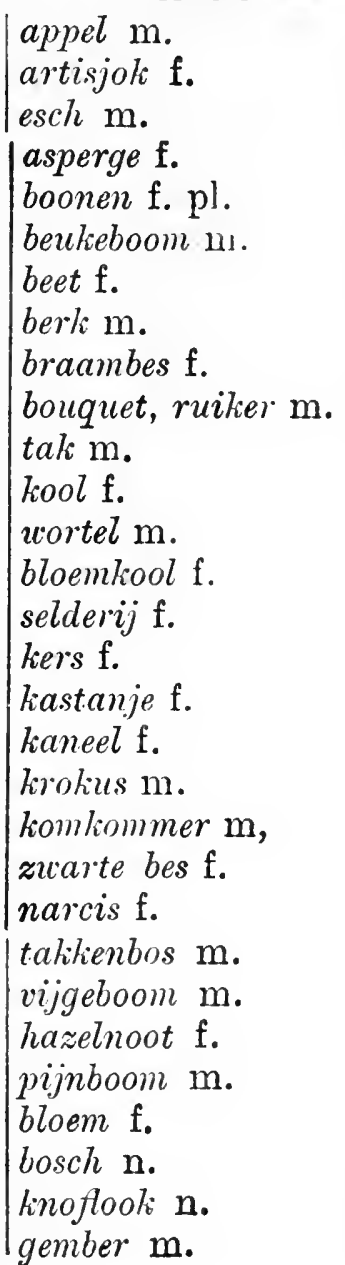 & $\begin{array}{l}\text { a(h)p'pe(r)l } \\
\text { a(h)rtisyok } \\
\text { ess } \\
\text { a(h)sspersye } \\
\text { boh'ne(r)n } \\
\text { bu(r)'ke(r)bohm } \\
\text { bayt } \\
\text { berrk } \\
\text { brahm'bess } \\
\text { booket', roy'ke(r)r } \\
\text { ta(h)k } \\
\text { kohl } \\
\text { worrtel } \\
\text { bloom'kohl } \\
\text { sel'de(r)rey } \\
\text { ke(r)rss } \\
\text { kahsta(h)n-ye(r) } \\
\text { kahnayl' } \\
\text { krolkkus } \\
\text { komkom'me(r)r } \\
\text { zwa(h)rte(r) bess } \\
\text { narsiss } \\
\text { ta(h)k'ke(r)nboss } \\
\text { feyge(r)'bohm } \\
\text { hahze(r)lnoht } \\
\text { peyn'bohm } \\
\text { bloom } \\
\text { boss } \\
\text { k-noflohk } \\
\text { gem'be(r)r }\end{array}$ \\
\hline
\end{tabular}




\begin{tabular}{|c|c|c|}
\hline English. & Dutch. & Pror \\
\hline $\begin{array}{l}\text { gooseberry } \\
\text { grapes (bunch of) } \\
\text { ivy } \\
\text { kernel } \\
\text { leaf } \\
\text { lemon } \\
\text { lettuce } \\
\text { lily } \\
\text { mace } \\
\text { melon } \\
\text { mulberry } \\
\text { myrtle } \\
\text { nettle } \\
\text { nut } \\
\text { oak-tree } \\
\text { parsley } \\
\text { pear } \\
\text { peas (green) } \\
\text { pine (tree) } \\
\text { pine-apple } \\
\text { pink } \\
\text { plum } \\
\text { potato } \\
\text { radishes } \\
\text { raspberry } \\
\text { root } \\
\text { rose } \\
\text { spinach } \\
\text { stalk (of a plant) } \\
\text { strawberry } \\
\text { stump (of a tree) } \\
\text { tree } \\
\text { tulip } \\
\text { turnip } \\
\text { vine } \\
\text { violet } \\
\text { walnut } \\
\text { watercress } \\
\text { wax } \\
\text { willow }\end{array}$ & 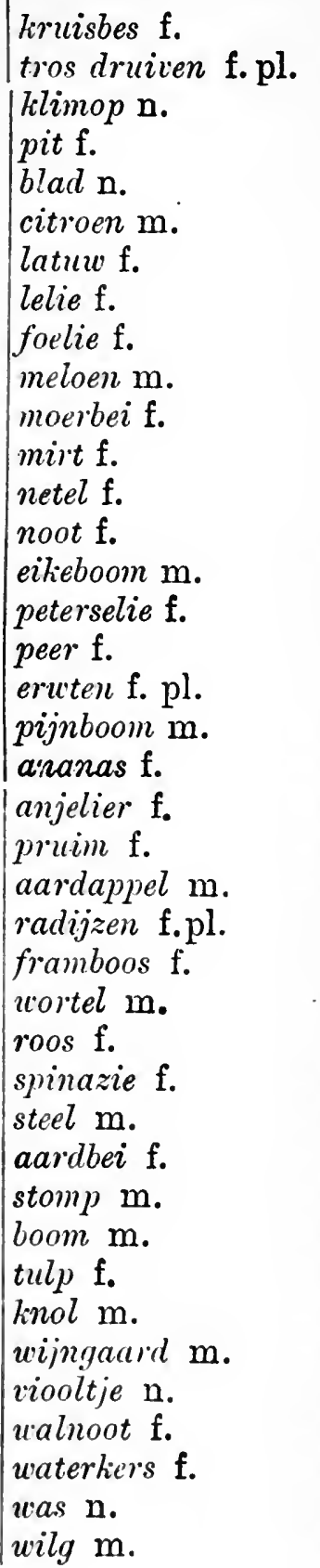 & $\begin{array}{l}\text { kroyss'bess } \\
\text { tross droyfe(r)n } \\
\text { klimop } \\
\text { pit } \\
\text { bla(h)t } \\
\text { sitroon } \\
\text { lahteuw } \\
\text { lay'lee } \\
\text { foo'lee } \\
\text { me(r)'loon } \\
\text { moor'bey } \\
\text { mir't } \\
\text { nayte(r)l } \\
\text { noht } \\
\text { eyke(r)'bohm } \\
\text { payte(r)rsay'lee } \\
\text { payr } \\
\text { erten } \\
\text { peybohm } \\
\text { ahnahna(h)s } \\
\text { a(h)nyeleer } \\
\text { proym } \\
\text { ahrd'a(h)p-pe(r)l } \\
\text { rahdey'ze(r)n } \\
\text { fra(ih)m'bohss } \\
\text { wor'te(r)l } \\
\text { rohss } \\
\text { spinahzee } \\
\text { stayl } \\
\text { ahr'd'bey } \\
\text { stomp } \\
\text { bohm } \\
\text { tulp } \\
\text { k-nol } \\
\text { weyn'gahrt } \\
\text { fee-ohlt'ye(r) } \\
\text { wa(h)l'nooht } \\
\text { wahte(r)rkerss } \\
\text { wa(h)ss } \\
\text { wilch }\end{array}$ \\
\hline
\end{tabular}


7. Colours. (Kleuren.)

(See Shopping, p. 109.)

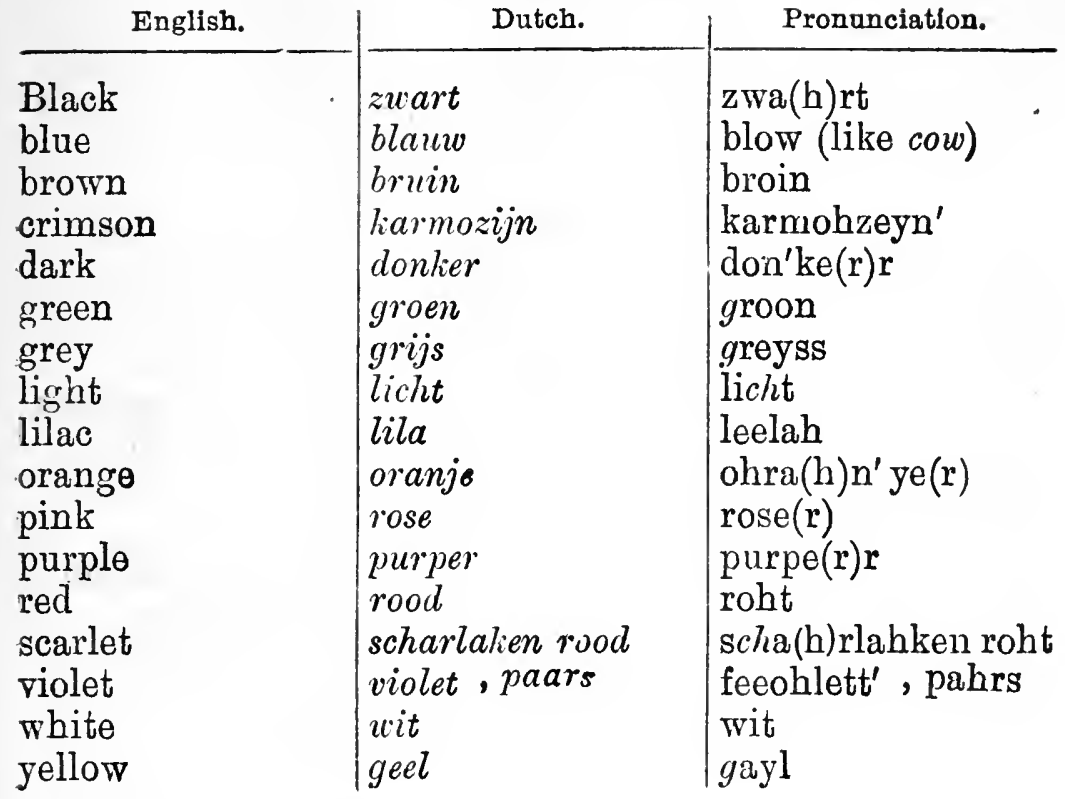

8. Times and Seasons. (Tijden en Jaarytijden.)

(For Conversations see page 108. )

Afternoon

birthday

Christmas

- Eve

dawn

day

day, to-

days of the week

Monday

Tuesday

Wednesday

Thursday

Friday

Saturday

Sunday namiddag $\mathrm{m}$.

verjaardag $\mathrm{m}$.

Kerstmis f.

Avond voor Kerstmis ahfont fohr kerrst-

dageraad $\mathrm{m}$.

dag $\mathrm{m}$.

heden, van daag

dagen van de weels

Maandag

Dinsdag

Woensdary

Donderdag

Vrijdag

Zaterdag

Zondag nahmid'da(h)ch fe(h)rjahrda(h)ch kerrstmiss dah'ye(r)raht [miss da(h)ch [dahg hayde $(\mathrm{r}) \mathrm{n}, \mathrm{fa}(\mathrm{h}) \mathrm{n}$ $\mathrm{da}(\mathrm{h}) g \mathrm{e}(\mathbf{r}) \mathrm{n}$ fa(h)n de(r) wayk mahn'da(h)ch dinșș'da(h)ch H oonss'da (h)ch don ie(r)rda(h)ch frey'da(h)ch zah'terda(h)ch zon'da(h)ch 


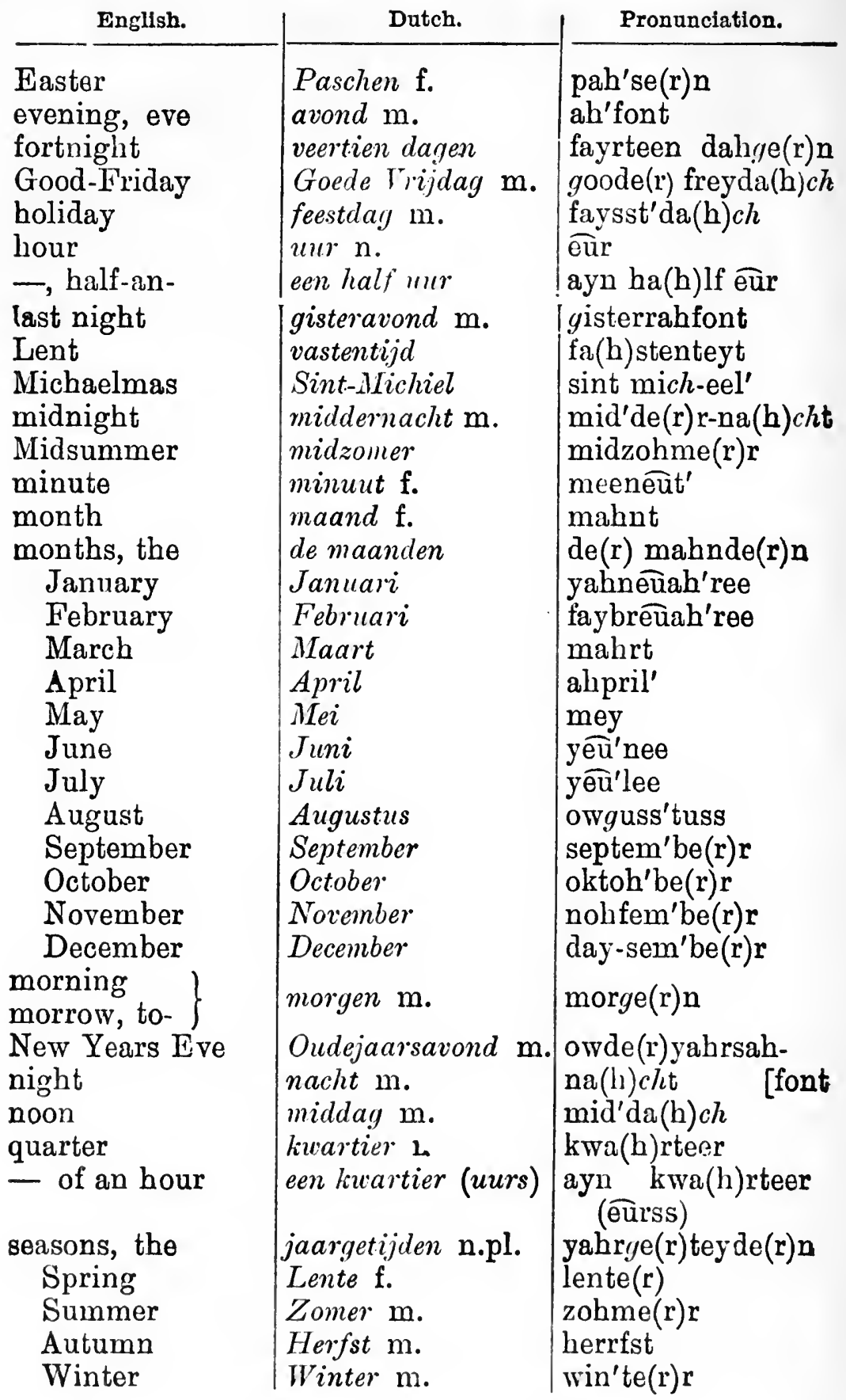




\begin{tabular}{|c|c|c|}
\hline English. & Dutch, & Pronunciation. \\
\hline second & seconde $\mathrm{f}$. & se $(r)$ konde $(r)$ \\
\hline sunrise & zonsopgang $\mathrm{m}$. & zonsop'ga(h)ng \\
\hline sunset & zonsondergang $\mathrm{m}$. & zonson'de (r)r- \\
\hline time & tijd $\mathrm{m}$ & teyt $\quad[g a(h) n g$ \\
\hline twilight & schemering f. & schayme(r)ring \\
\hline week & week $\mathrm{f}$. & wayk \\
\hline week-day & werkdag $\mathrm{m}$. & werrkda(h)ch \\
\hline weekly & welelijksch & wayke(r)le(r) ks \\
\hline Whitsuntide & Pinksteren f. & pinkste(r)ren \\
\hline $\begin{array}{l}\text { year } \\
\text { yesterday }\end{array}$ & $\begin{array}{l}\text { jaar } \mathbf{n} . \\
\text { gisteren }\end{array}$ & $\begin{array}{l}\text { yahr } \\
\text { yiste(r)ren }\end{array}$ \\
\hline - morning & - morgen & - mor $^{\prime} g \mathrm{e}(\mathrm{r}) \mathrm{n}$ \\
\hline - evening & - avond & - ah'font \\
\hline
\end{tabular}

9. Holidays in Holland.

(Algemeen erkende Feestdayen.)

Ascension Day Hemelvaartsdag m. hay'me(r)lfahrts-

Christmas Day

Easter Day

Good Friday

New Year's Day

Whitsunday

$\mid \begin{aligned} & \text { Hemelvaartsdag m. } \\ & \text { Kerstdag m. } \\ & \text { Paaschdag m. } \\ & \text { Goede Vrijdag m. } \\ & \text { Nieuwjaarsdag m. } \\ & \text { Pinksterdag m. }\end{aligned}$

$$
\mathrm{da}(\mathrm{h}) \mathrm{ch}
$$

kerrst'da(h)ch

palıss'da(h)ch

goode(r) freyda(h)ch

nee'w - yahrss$\mathrm{da}(\mathrm{h}) \mathrm{ch}$

pinkste(r)rda(h)ch

\section{Town and Country. (Stad en Land.)}

(For Conversations see page 108.)

Bank (mound)
barn
bridgc
brook
building (edifice)
bush
cathedral
country
courtyard
custom-house
dale
ditch
environs

\begin{tabular}{|l|l} 
oever m. & oo'fe(r)r \\
schuur f. & scheur \\
brug f. & bruch \\
beek f. & bayk \\
gebouw n. & ge(r)bow' \\
kreupelhout. n. & kru(r)pelhowt \\
domkerk f. & dom'kerrk \\
land n. & la(h)nt \\
binnenplaats f. & binne(r)nplahtss \\
douanenkantoor n. & dooahnenka(h)n- \\
dal n. & da(h)l \\
sloot f. & sloht \\
omstreken f.pl. & om'strayken
\end{tabular}




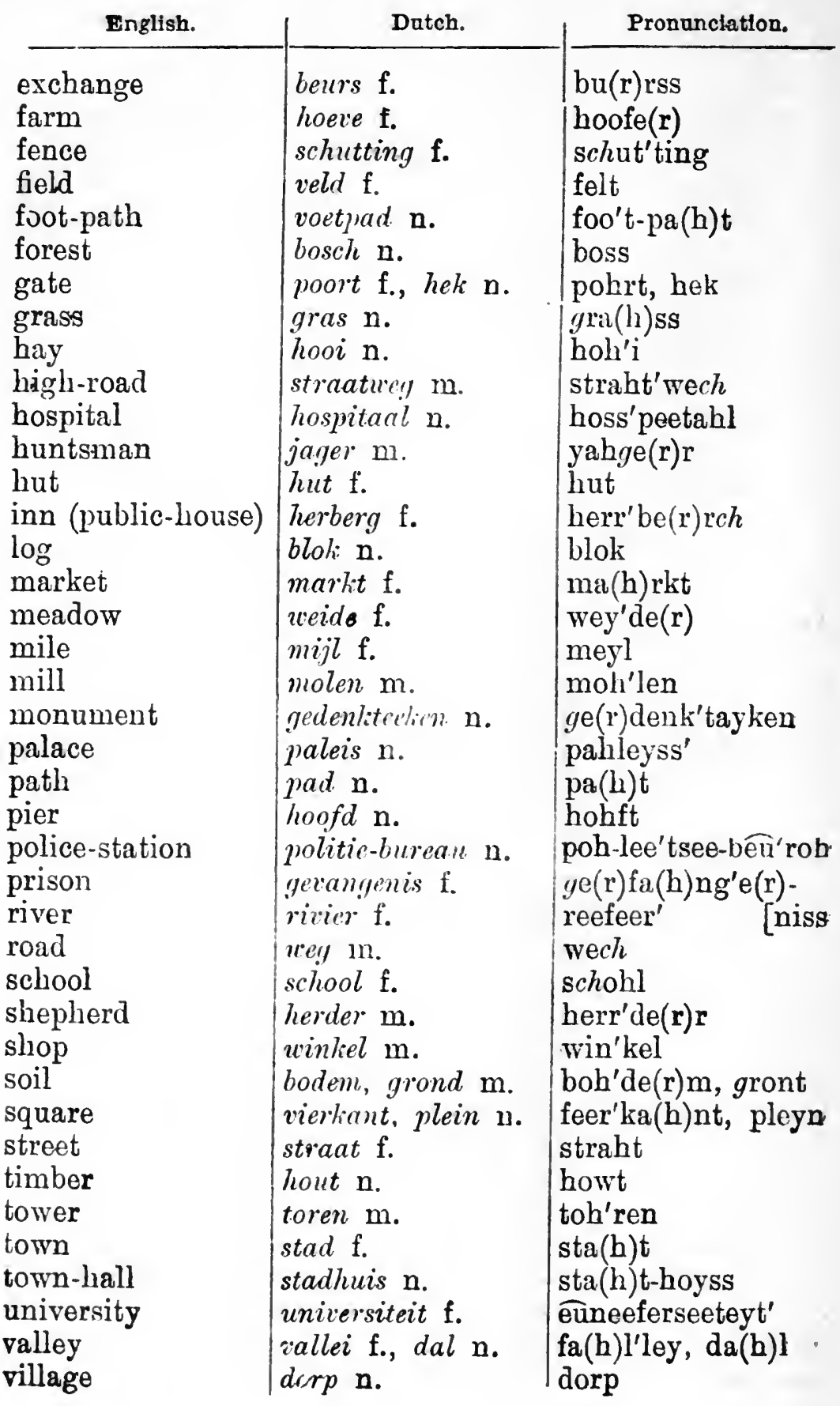




\begin{tabular}{|c|c|c|}
\hline English. & Dutch. & Pronunciation. \\
\hline $\begin{array}{l}\text { waterfall } \\
\text { wood (trees, etc.) }\end{array}$ & $\begin{array}{l}\text { waterval } \mathrm{m} . \\
\text { woud } \mathrm{n} .\end{array}$ & $\begin{array}{l}\text { wahte }(\mathrm{r}) \mathrm{rfa}(\mathrm{h}) \mathrm{l} \\
\text { wowt }\end{array}$ \\
\hline
\end{tabular}

\section{Mankind: Relations.}

(Menschelijli geslacht: Familiebetreklingen.)

Aunt

bachelor

boy

bride

bridegroom

brother

child

cousin

daughter

daughter-in-law

family

father

father-in-law

girl

granddaughter

grandfather

grandmother

grandson

guardian

heir

husband

man

mother

mother-in-law

nephew

niece

parents

sister

son

son-in-law

uncle

widow

widower

wife

woman tante $\mathrm{f}$.

"rijgezel m.

jongen $\mathrm{m}$.

bruid $\mathrm{f}$.

bruidegom $\mathrm{m}$.

broeder $\mathrm{m}$.

kind $\mathrm{n}$.

neef m., nicht $\mathrm{f}$.

dochter $\mathrm{m}$.

schoondochter f.

gezin $\mathrm{n}$.

vader $\mathrm{m}$.

schoonvader $\mathrm{m}$.

meisje $\mathrm{n}$.

kleindochter $\mathrm{f}$.

grootvader $\mathrm{m}$.

grootmoeder f.

kleinzoon $\mathrm{m}$.

voogd $\mathrm{m}$.

erfyenaam m.

man, echtgenoot m. $\mathrm{ma}(\mathrm{h}) \mathrm{n}$, echt'ge(r)-

mensch, man $\mathrm{m}$.

moeder f.

schoonmoeder f.

neef $\mathrm{m}$.

nicht $\mathrm{f}$.

ouders m.\&f.pl.

zuster $\mathrm{f}$.

zoon $\mathrm{m}$.

schoonzoon $\mathrm{m}$.

oom $\mathrm{m}$.

weduwe $\mathrm{f}$.

weduwnaar $\mathrm{m}$.

vrouw, echtgenoote f. frow, echt'ge(r)noh-

vrouw $\mathrm{f}$.

ta(h)n'te(r)
freyge(r)zell
yo(h)ngen
broyt
broyde(r)gom
brooder(r)
kinnt
nayf, nicht
dochte(r)r
schohn'dochte(r)r
ge(r)zin
fah'de(r)r
schohn'fahde(r)r
meyssye(r)
kleyn'dochte(r)r
groht'fahde(r)r
groht'moode(r)r
kleyn'zohn
fohcht
erf'genahm
ma(h)n, echt'ge(r)-
menss, ma(h)n
moode(r)r
schohn'moode(r)r
nayf
nicht
owde(r)rss
zusste(r)r
zohn
schohnzohn
ohm
way'deuwe(r)
way'deunahr
frow, echt'ge(r)noh-
frow


12. The Human Body. (Het Menschelijk Lichaam.)

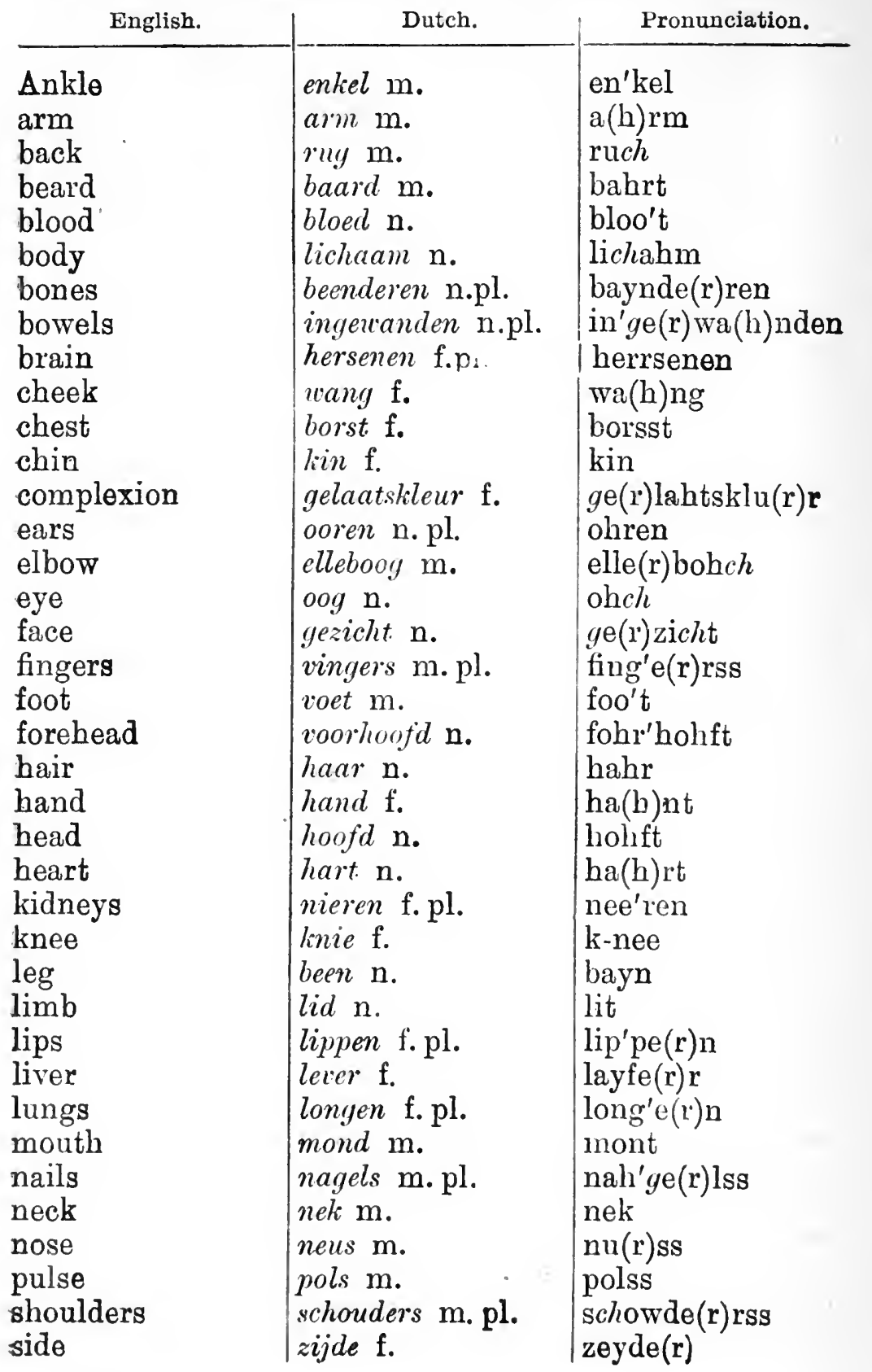




\begin{tabular}{l|l|l}
\multicolumn{1}{c|}{ English. } & \multicolumn{1}{c}{ Dutch. } & \multicolumn{1}{c}{ Pronunciation. } \\
\hline skin & huid $\mathrm{f}$. & hoyt \\
spine & ruggegraat f. & ragge(r)graht \\
stomach & maag $\mathrm{f}$. & mahch \\
throat & keel $\mathrm{f}$. (interior) $;$ & kayl, ha(h)ls \\
thumb & duim $\mathrm{m} . \quad$ hals $\mathrm{m}$. & doym \\
toe & teen $\mathrm{m} . \quad$ & tayn \\
tongue & tong $\mathrm{f}$. & tong \\
tooth & tand $\mathrm{m}$. & ta(h)nt
\end{tabular}

\section{Health. (Gezondheid.)}

Accident ambulance apoplexy bandage biliousness bite (of a dog) blister bruise burn to catch a disease

contagion chemist's shop chill to catch a chill chilblains cough corn cramp cure deaf dentist diarrhœa diet doctor draught

dumb exerciso
(For Conversations see page 107.)

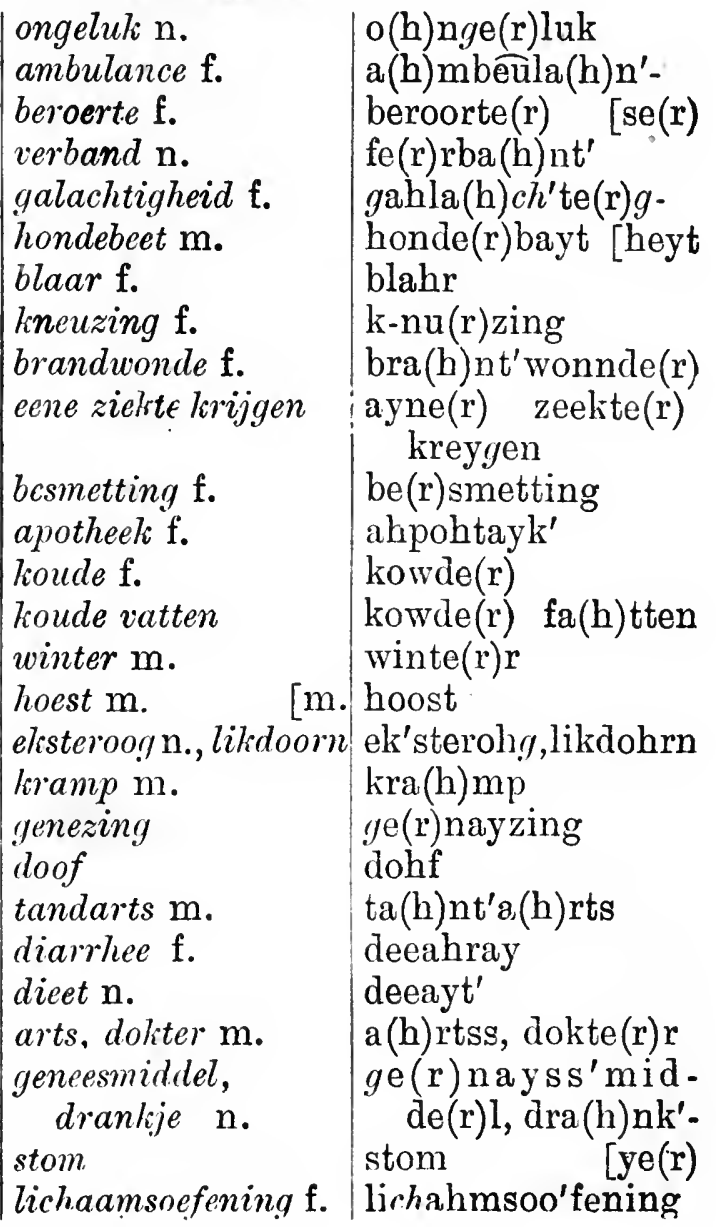




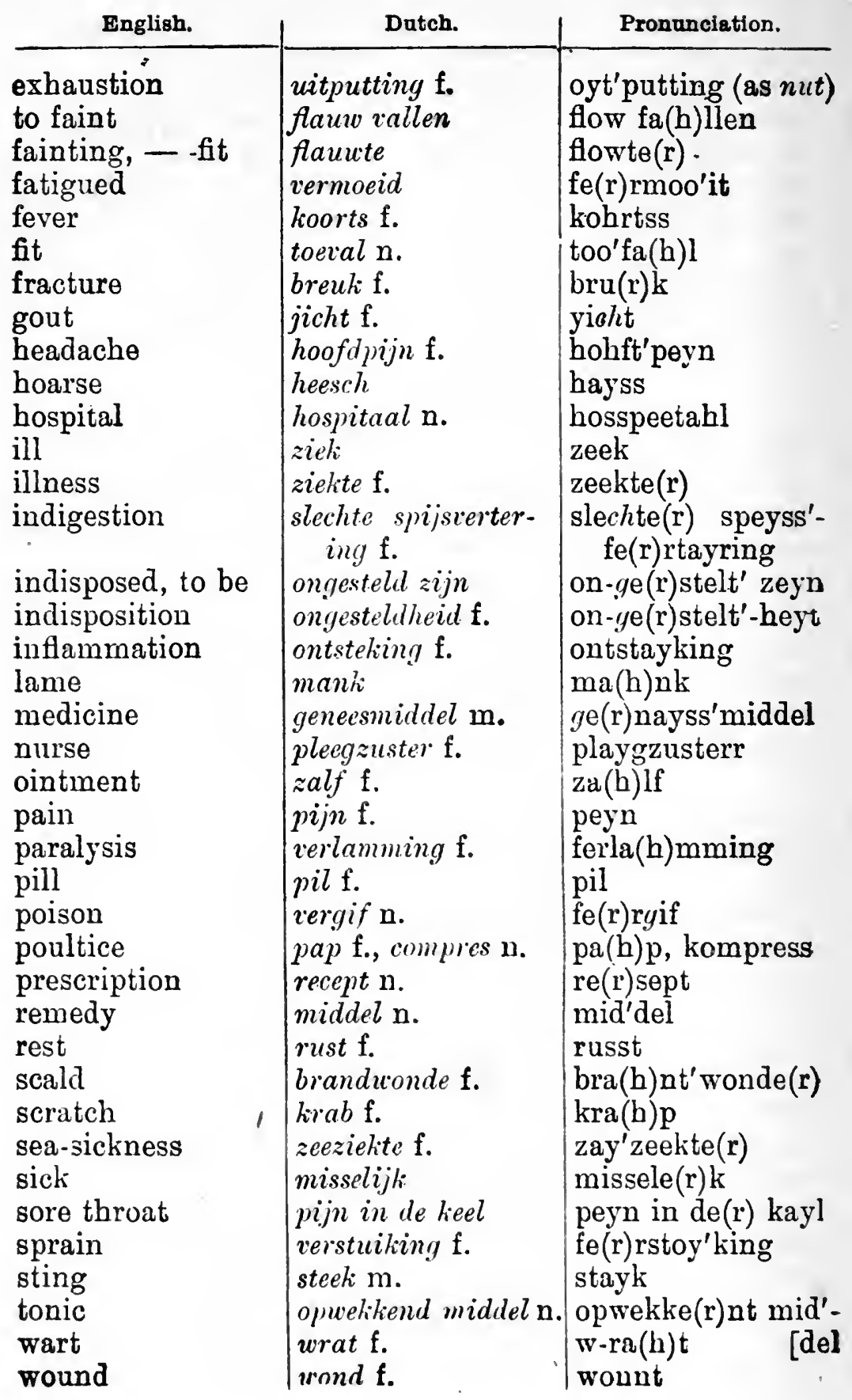




\section{Dress. (Kleeding.;}

(Bee Shopping, Dressmaker, Shoemaker, Laundress, \&c., pages 109 to 112.)

\begin{tabular}{|c|c|c|}
\hline Er & Dutch. & Pronunciation. \\
\hline $\begin{array}{l}\text { Blouse (lady's) } \\
\text { bonnet } \\
\text { boots } \\
\text { bracelet } \\
\text { braces } \\
\text { brooch } \\
\text { brush } \\
\text { buttons } \\
\text { button-hook } \\
\text { cap } \\
\text { cloak } \\
\text { slothes-brush } \\
\text { coat } \\
\text { collar } \\
\text { comb } \\
\text { cotton } \\
\text { drawers } \\
\text { dress } \\
\text { gaiters } \\
\text { garters } \\
\text { glove } \\
\text { hair-pin } \\
\text { handkerchief } \\
\text { hat } \\
\text { lace } \\
\text { lining } \\
\text { mackintosh } \\
\text { necklace } \\
\text { needle } \\
\text { overcoat } \\
\text { parasol } \\
\text { petticoat } \\
\text { pin } \\
\text { pocket } \\
\text { purse } \\
\text { ring } \\
\text { shirt } \\
\text { brom }\end{array}$ & 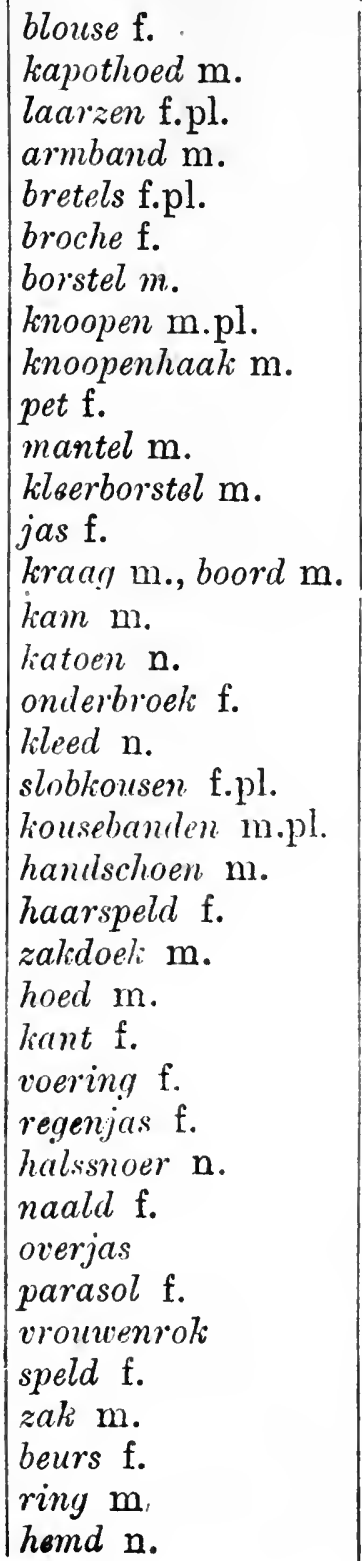 & $\begin{array}{l}\text { blooze } \\
\text { kahpo(h)thoot } \\
\text { lahrze(r)n } \\
\text { a(h)rm'ba(h)nt } \\
\text { bretells } \\
\text { bro(h)sh } \\
\text { borste(r)l } \\
\text { k-nohpe(r)n } \\
\text { k-nohpe(r)nhahk } \\
\text { pet } \\
\text { ma(h)nte(r)l } \\
\text { klay'rborste(r)l } \\
\text { yass } \\
\text { krahch, bohrt } \\
\text { ka(h)m } \\
\text { kahtoon' } \\
\text { on'de(r)rbroo-k } \\
\text { klayt } \\
\text { slop'kowssen } \\
\text { kow'se(r)ba(h)nden } \\
\text { ha(h)nt'schoon } \\
\text { hahr-spelt } \\
\text { za(h)k'dook } \\
\text { hoot } \\
\text { ka(h)nt } \\
\text { fooring } \\
\text { ray'ge(r)nya(h)ss } \\
\text { ha(h)lssnoor } \\
\text { nahlt } \\
\text { oh'fe(r)r-ya(h)ss } \\
\text { pa(h)rahsol } \\
\text { frowe(r)nrok } \\
\text { spelt } \\
\text { za(h)k } \\
\text { bu(r)rss } \\
\text { ring } \\
\text { hemt }\end{array}$ \\
\hline
\end{tabular}




\begin{tabular}{|c|c|c|}
\hline English. & Dutch. & Pronnnciation. \\
\hline $\begin{array}{l}\text { shoe } \\
\text { shoe-horn } \\
\text { skirt } \\
\text { slipper } \\
\text { stays (corset) } \\
\text { stockings } \\
\text { thread } \\
\text { tie } \\
\text { trousers } \\
\text { umbrella }\end{array}$ & $\begin{array}{l}\text { schoen } \mathrm{m} . \\
\text { schoenhoren } \mathrm{m} . \\
\text { japonrok } \mathrm{m} . \\
\text { pantoffel } \mathrm{f} . \\
\text { corset } \mathrm{n} . \\
\text { kousen } \mathrm{f} . \mathrm{pl} . \\
\text { draad } \mathrm{m} . \\
\text { das } \mathrm{f} . \\
\text { broek } \mathrm{f} . \\
\text { paraplu } \mathrm{f} .\end{array}$ & $\begin{array}{l}\text { schoon } \\
\text { schoonhohre(r)n } \\
\text { yahpo(h)nrok } \\
\text { pa(h)ntoffel } \\
\text { korset' } \\
\text { kowsse(r)n } \\
\text { draht } \\
\text { da(h)ss } \\
\text { broo'k } \\
\text { pahrahplou' }\end{array}$ \\
\hline $\begin{array}{l}\text { veil } \\
\text { waistcoat } \\
\text { watch }\end{array}$ & $\begin{array}{l}\text { sluier m., voile } \mathrm{f} . \\
\text { vest } \mathrm{n} . \\
\text { horioge } \mathrm{n} .\end{array}$ & $\begin{array}{l}\text { sloy'}^{\prime} \theta(r) r, \text { fwawle }(r) \\
\text { fest } \\
\text { horloh'ge(r) }\end{array}$ \\
\hline
\end{tabular}

15. Food and Drink. (Voedsel en Dranken.)

(See also pages 14 to 18, and for Conrersations see pages 104 to 106.)

Bacon

beef

beef-steak

beer

bread

-, brown

-, new

-, stale

-, white

breakfast

butter

cake

cheese

chocolate

cigar

coffee

cream

dinner

eggs

fat

flour

gin

ham spete $\mathrm{n}$. vundrleesch $\mathrm{n}$.

biefstuk $\mathrm{m}$.

bier $\mathrm{n}$.

brood $\mathrm{n}$.

roggebrood

versch brood

oulbaliken brood

wittebrood

ontbijt $\mathrm{n}$.

boter $\mathrm{f}$.

koek in., tulliand

kaas f.

chocolade $\mathrm{f}$.

sigaar f.

kolfie $\mathrm{f}$.

room $\mathrm{m}$.

middagmaal $\mathbf{n}$.

eieren n. pl.

vet $\mathrm{n}$.

meel $\mathrm{n}$.

jenever m.

ham $\mathrm{m}$. spek

runtflayss

beef'stuk

beer

broht

roy'ye(r)(broht

ferss'broht

owdba(h) k'ke(r)n-

witte(r)brobt [broht ontbeyt'

bohte(r)r

koo'k, tulba(h)nt

kalıss

chokolahde(r)

seegahr

kof'fee

rohm

mid'da(h)gmahl

ey'e(r)re(r)n

fet

mayl

ye(r)nayfe (r)r

$\mathrm{ha}(\mathrm{h}) \mathrm{m}$ 


\begin{tabular}{|c|c|c|}
\hline & tch. & onanciation. \\
\hline $\begin{array}{l}\text { honey } \\
\text { ice } \\
\text { jam } \\
\text { kidneys } \\
\text { lamb } \\
\text { lean } \\
\text { lemonade } \\
\text { meat } \\
\text {-, boiled } \\
\text {-, roast } \\
\text { milk } \\
\text { mustard } \\
\text { mutton } \\
\text { - cutlets } \\
\text { oil } \\
\text { omelet } \\
\text { onion } \\
\text { pepper } \\
\text { pork } \\
\text { pudding } \\
\text { rice } \\
\text { roll } \\
\text { salad } \\
\text { salt } \\
\text { sausage } \\
\text { soda water } \\
\text { soup } \\
\text { sugar } \\
\text { supper } \\
\text { syphon } \\
\text { tea } \\
\text { thirst } \\
\text { tobacco } \\
\text { under-done } \\
\text { veal } \\
\text { vegetables } \\
\text { vinegar } \\
\text { water } \\
\text { wolldono }\end{array}$ & 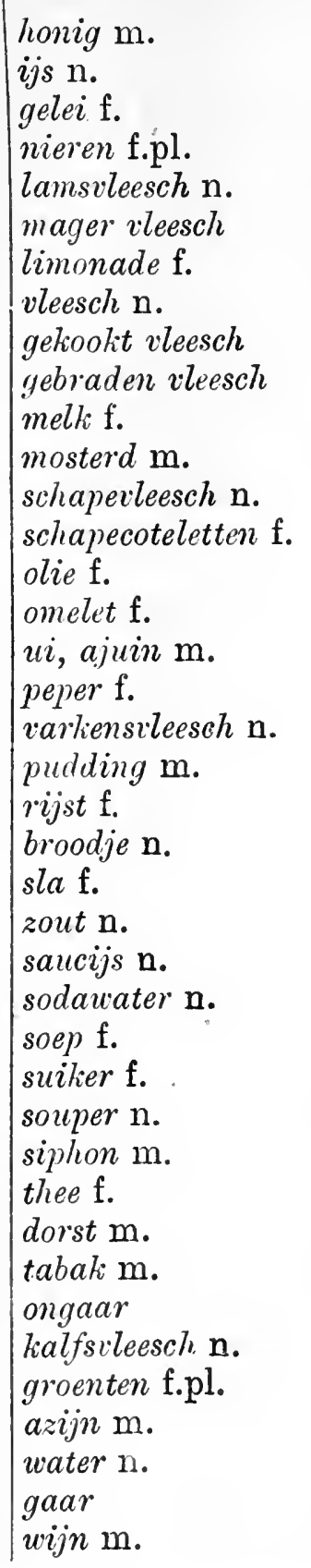 & $\begin{array}{l}\text { hohnig } \\
\text { eyss } \\
\text { she(r)ley } \\
\text { neere(r)n } \\
\text { la(h)msflayss } \\
\text { mahge(r)r flayss } \\
\text { leemonah'de(r) } \\
\text { flayss } \\
\text { ge(r)kohkt flayss } \\
\text { ge(r)brahden flayss } \\
\text { mel'k } \\
\text { mossterrt } \\
\text { schah'pe(r)flayss } \\
\text { schah'pe(r)kohte(r)- } \\
\text { ohlee } \\
\text { o(h)melet lette(r)n } \\
\text { oy, ahyoin' } \\
\text { paype(r)r } \\
\text { fa(h)r'ke(r)nsflayss } \\
\text { pe(r)d'ding } \\
\text { reysst } \\
\text { broht'ye(r) } \\
\text { slah } \\
\text { zowt } \\
\text { sohseyss' } \\
\text { soh'dahwahte(r)r } \\
\text { soop } \\
\text { soyke(r)r } \\
\text { soopay } \\
\text { seefo(h)n } \\
\text { tay } \\
\text { dorst } \\
\text { tahba(h)k' } \\
\text { o(h)ngahr } \\
\text { ka(h)lfs'flayss } \\
\text { groonte(r)n } \\
\text { ahzeyn' } \\
\text { wahte(r)r } \\
\text { gahr } \\
\text { weyn }\end{array}$ \\
\hline
\end{tabular}


16. House and Furniture. (Huis en Huisraad.) (For Shopping, see p. 109.)

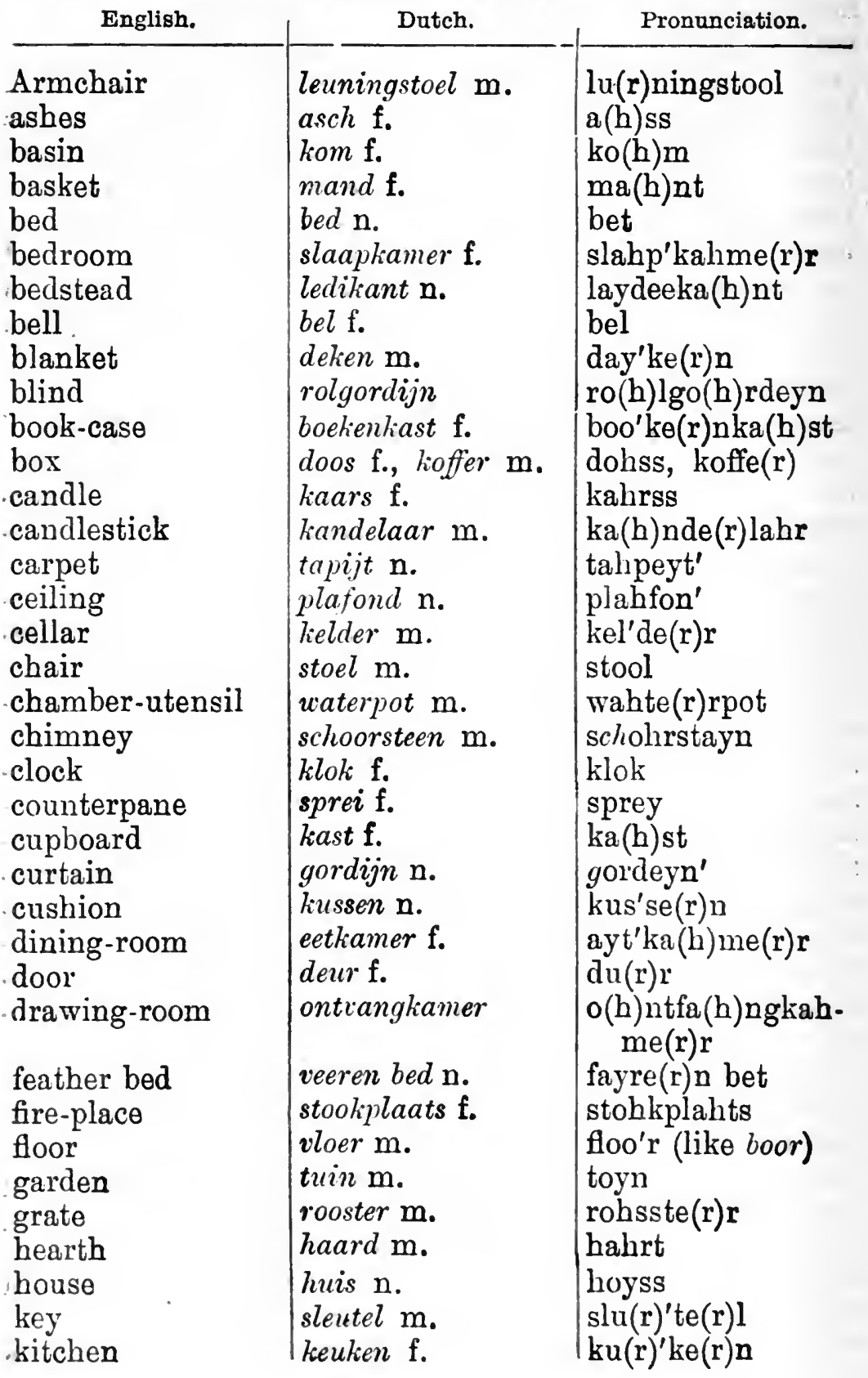




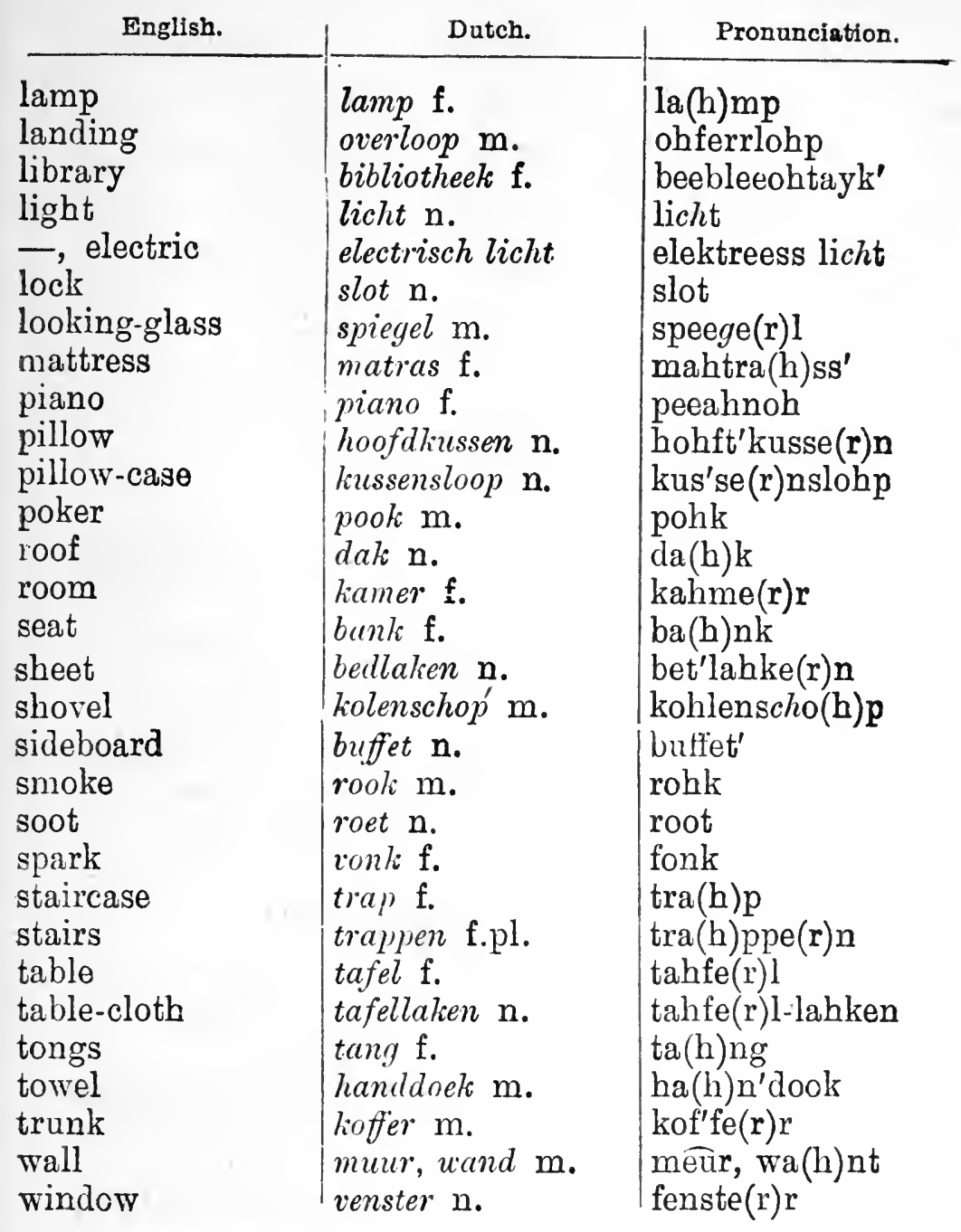

17. Cooking and Eating Utensils.

(Keukengereedschappen.)

(For Shopplng, see p. 100.)

Coals

coffee-pot

cup

decanter

dish

firewood

kolen f.pl.
kofiekan f.
kop m.
karaf f.
schotol m.
brandhout n.

koh'le(r)n

koffeeka(h)n

kop

kahra(h) $\mathrm{f}^{\prime}$

schoh'te(r)l

bra(h)nt'howt 


\begin{tabular}{|c|c|c|}
\hline English. & Dutch. & Pronunciation. \\
\hline fork & vork f. & forrk \\
\hline frying-pan & braadpan f. & brahtpa(h)n \\
\hline gas & gas $\mathrm{n}$. & $g a(h) s s$ \\
\hline glass & glas n. & $g l a(h) s s$ \\
\hline gridiron & rooster $\mathrm{m}$. & rohsste(r)r \\
\hline kettle & ketel m. & kaytel \\
\hline knife & mes $\mathrm{n}$. & mess \\
\hline & deksel $\mathrm{n}$. & $\operatorname{dekse}(\mathrm{r}) 1$ \\
\hline matches & lucifer $\mathrm{m}$. & leuseefehr \\
\hline oven & oven $\mathrm{m}$. & ohfe(r)n \\
\hline plate & bord $\mathrm{n}$. & bort \\
\hline saucepan & stoofpan $\mathbf{f}$. & stohfpa(h)n \\
\hline saucer & schoteltje n. & schoh'te(r)lt-ye(r) \\
\hline serviette & servet $\mathrm{n}$. & serrfet' \\
\hline soup ladle & soeplepel m. & soop'laype(r)l \\
\hline spoon & lepel $\mathrm{m}$. & laype $(\mathrm{r}) 1$ \\
\hline stove & kachel f. & $\mathrm{ka}(\mathrm{h}) \operatorname{ch} \mathrm{e}(\mathrm{r}) \mathrm{l}$ \\
\hline table-cloth & tafellatien $\mathrm{n}$. & tahfe(r)l-lahke(r)n \\
\hline $\begin{array}{l}\text { tea-pot } \\
\text { tray }\end{array}$ & $\begin{array}{l}\text { theepot, trekpot m. } \\
\text { blaadje } \mathrm{n} .\end{array}$ & $\begin{array}{l}\text { taypot, trekpot } \\
\text { blahd-ye(r) }\end{array}$ \\
\hline
\end{tabular}

18. Religion. (Godsdienst.)

(See Vocabulary 9, p. 21.)

\begin{tabular}{|c|c|c|}
\hline $\begin{array}{l}\text { Aisle } \\
\text { altar } \\
\text { baptism } \\
\text { belfry } \\
\text { Bible Society } \\
\text { cemetery } \\
\text { chancel } \\
\text { chapel } \\
\text { choir } \\
\text { choir-master } \\
\text { church } \\
\text { clergy } \\
\text { clergyman } \\
\text { Communion } \\
\text { confirmation } \\
\text { donation }\end{array}$ & $\begin{array}{l}\text { zijbeut } \mathrm{m} . \\
\text { altaar } \mathrm{n} . \\
\text { doop } \mathrm{m} . \\
\text { klokkentoren } \mathrm{m} . \\
\text { Bijbelgenootschap } \\
\mathrm{n} . \\
\text { begraafplaats } \mathrm{f} . \\
\text { koor } \mathrm{n} . \\
\text { kapel } \mathrm{f} . \\
\text { koor } \mathrm{n} . \\
\text { koormeester } \mathrm{m} . \\
\text { kerk } \mathrm{f} \\
\text { geestelijkheid } \mathrm{f} . \\
\text { geestelijke } \mathrm{m} . \\
\text { avondmaal } \mathrm{n} . \\
\text { bevestiging } \mathrm{f} . \\
\text { schenking } \mathrm{f} .\end{array}$ & $\begin{array}{l}\text { | seybu(r)k } \\
\text { a(h)l'tahr } \\
\text { dohp } \\
\text { klok'kentohre(r)n } \\
\text { bey'be(r)lgenoht- } \\
\text { scha(h)p } \\
\text { be(r)grahf-plahts } \\
\text { kohr } \\
\text { kahpell' } \\
\text { kohr } \\
\text { kohr-maysste(r)r } \\
\text { kerk } \\
\text { gayss'te(r)le(r)kheyt } \\
\text { gayss'te }(\mathrm{r}) \mathrm{le}(\mathrm{r}) \mathrm{ke}(\mathrm{r}) \\
\text { ah'font-mahl } \\
\text { be(r)feste(r)ging } \\
\text { schenking }\end{array}$ \\
\hline
\end{tabular}




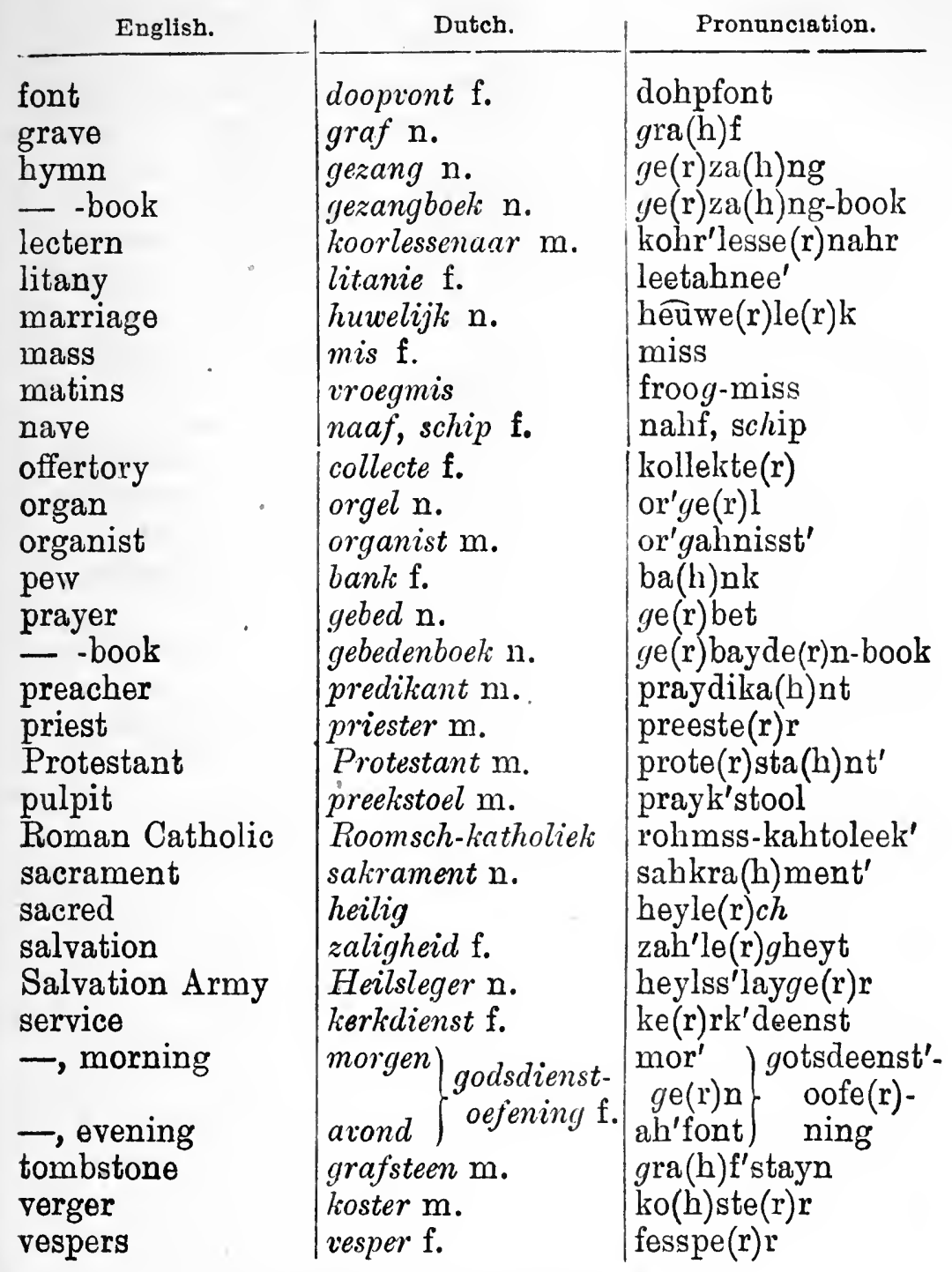

19. Legal and Judicial Terms. (Wettelijke en Rechterlijke Termen.)

Acquittal agreement allegation allege, to apology \begin{tabular}{|l|l} 
vrijspraak i. & freysprahk \\
overeenkomst $\mathrm{f}$. & oh'feraynko(h)mst \\
verklaring $\mathrm{f}$. & fe(r)rklahring \\
beweren & be(r)wayre(r)n \\
verontschuldiging $\mathrm{f}$. & fe(r)ront'schulde(r)-
\end{tabular} ging 


\begin{tabular}{l}
\multicolumn{1}{c}{ Euglish. } \\
arrest to \\
attest, to \\
attestation \\
attorney \\
-, power of \\
authorise, to \\
award \\
bail \\
bailiff \\
bond (for loan) \\
burglary \\
case for the prose- \\
cution
\end{tabular}

- for the defence

charge (accusation) aanklacht $\mathrm{f}$.

-, to

claimant

client

complaint

conviction

court of justice

deed (of sale)

- of assignment

defence

defend (in court)

defendant (in a suit) deposition(on oath)

effects (goods)

equity

estate

-, real and

personal

evidence

excise

fee (of office)

beschuldigen

eischer $\mathrm{m}$.

cliënt $\mathrm{m}$.

aanklager $\mathrm{m}$.

schuldigverklaring $\mathrm{f}$.

rechtbank f.

verkoopcontract $\mathrm{n}$.

akte van afstand $\mathrm{f}$.

verdediging $\mathrm{f}$.

verdedigen

verweerder $m$.

verklaring $\mathrm{f}$.

goederen n.pl.

billijheid f.

boedel $\mathrm{m}$.

roerend en onroer.

end goed $\mathrm{n}$.

Pronunciation.

in bechte(r)niss

nayme $(r) n$

ferklahrre(r)n

ferklahring

a(h)tfohkahnt'

fol'ma(h)cht, proh-

kêrah'tee

ma(h)chtige(r)n

be(r)slissing

borg'tocht

du(r)rwabrde(r)r

$o(h) n d e r r p a(h) n t$

inbrahk

ahn'kla(h)cht

fe(r)rday'de(r)ging

ahn'kla(h)cht be(r)schulde (r)-

eysser

klee-ant'

$[g e(r) n$

ahn'klahyer [ring

schul'de(r)gferklah-

rechtba(h)nk

ferkohp'kontra(h) kt

$a(h) k t e(r) \quad f a(h) n$

$a(h) f^{\prime} \operatorname{sta}(h) n t$

fe(r)rdayde(r)ging

fe(r)rdayde (r)ge(r)n

fe(r)rwayrde(r)

fe(r)rklahring

goo'dere(r)n

bil'le(r)kheyt

boode(r)l

roore(r)nt en on'.

roore(r)nt goo't

getuigenis f., bewijs ge(r)toygeniss, be-

accijns $\mathrm{m}$. weyss

a(h) kseynss'

salaris $\mathrm{n}$. 


English.
fine (payment in
forgery [money)
illegal
information
injunction
injustice
inventory
jail
judge
judgment, to give
justice of the peace
law, civil
-, criminal
- -suit
lawyer
lender
non-suit, to
oath
-, to take an
parties (in a suit)
penal
perjury
petitioner
police
- office
proceedings (legal)

$\frac{\text { Dutch. }}{\text {. }}$

boete $\mathrm{f}$.

vervalsching f. fe(r)rfa(h)lsing

onvettig

aanbrenging f. ahnbrenging

bevel van schorsing be(r)fell ve(h)n-

$\mathrm{n}$.

onvecht $\mathrm{n}$.

inventaris $\mathrm{f}$.

!levangenis $\mathrm{f}$.

rechter $\mathrm{m}$.

uitspraak doen

vrederechter $\mathrm{m}$. frayde(r)rechite(r)r

burgerlijk wetboek $\mathrm{n}$. burgerle(r)k wetstrafuet $\mathrm{f}$. rechtsgeding $\mathrm{n}$.

rechtsgeleerde $\mathrm{m}$.

leener $\mathrm{m}$.

afwijzen

eed $\mathrm{m}$.

een eed doen

partijen

lijfstraffelijk

meineed $\mathrm{m}$.

requestrant $\mathrm{m}$.

politie f.

politie-bureau

onrecht

inventahriss

$g \mathrm{e}(\mathrm{r}) \mathrm{fa}(\mathrm{h}) \mathrm{ng}^{\prime} \mathrm{e}(\mathrm{r})$ -

rechter [niss

oytsprahk doon stra(h)fwet [boo'k recht'sge(r)ding rechtsge(r)layrde (r)

layne(r)r

a(h)fweyze(r)n ayt

ayn ayt doon

pa(h)rtey'en

leyfstra(h)ffe(r) -

meynayt $\quad[l e(r) k$

raykwestra $(\mathrm{h}) \mathrm{nt}$

pohleetsee

- -b جurroh

prohsees

proof

prosecution

court)

prosecutor

punishment

release

robbery

seal

-, to

sentence

statement

sue, to

suit

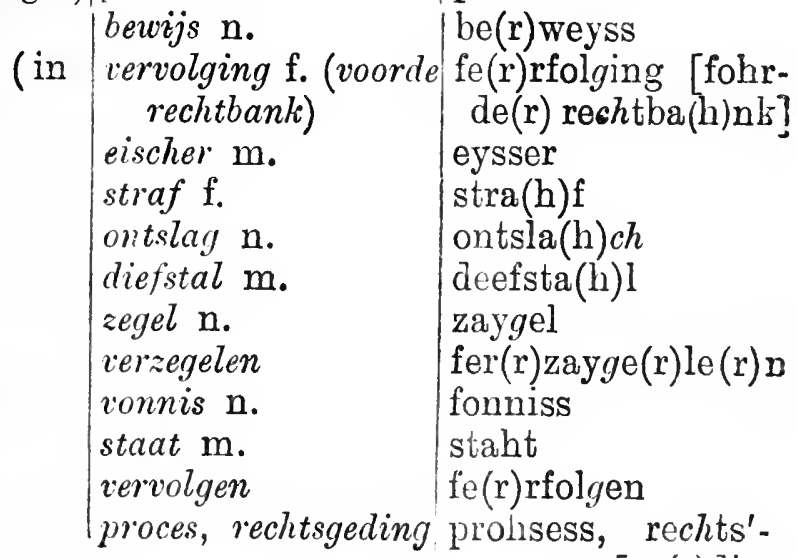




\begin{tabular}{|c|c|c|}
\hline English. & Dutch. & Pronunclation. \\
\hline summons (of court) & dagvaarding $\mathrm{f}$. & $\mathrm{da}(\mathrm{h}) g^{\prime}$ fahrd \\
\hline tenant (of house) & huurder $\mathrm{m}$. & heurde $(r) r$ \\
\hline testament (will) & uiterste uil, testa- & oytersste(r) will, \\
\hline & ment $\mathrm{m}$ & tesstahment \\
\hline testator & erflater $\mathrm{m}$. & e(r)rflahte $(r) r$ \\
\hline theft & diefstal m. & $\operatorname{deefsta}(\mathrm{h}) l$ \\
\hline thief & dief $\mathrm{m}$ & deef \\
\hline trial by $\mathrm{j}$ & $\begin{array}{r}\text { gerechtelijk onder- } \\
\text { zoek door de jury }\end{array}$ & $\begin{array}{l}g \mathrm{e}(\mathrm{r}) \mathrm{rechte}(\mathrm{r}) \mathrm{le}(\mathrm{r}) \mathrm{k} \\
\text { onderzook dohr }\end{array}$ \\
\hline & & de(r) yêury \\
\hline uniawful & onwettig & onwette $(\mathrm{r}) \mathrm{ch}$ \\
\hline valid & geldig & gelde(r)ch \\
\hline witness & getuige m.\& f. & $g e(r)$ toyge $(r)$ \\
\hline
\end{tabular}

20. Professions \& Trades. (Beroepen en Ambachten.) (For Shopping, see p. 108.)

\begin{tabular}{|c|c|c|}
\hline $\begin{array}{l}\text { eller } \\
\text { r } \\
\text { er } \\
\text { ater } \\
\text { ist } \\
\text { man } \\
\text { man } \\
\text { ercial travel- } \\
\text { ctioner } \\
\text { maker } \\
\text { man } \\
\text { t }\end{array}$ & 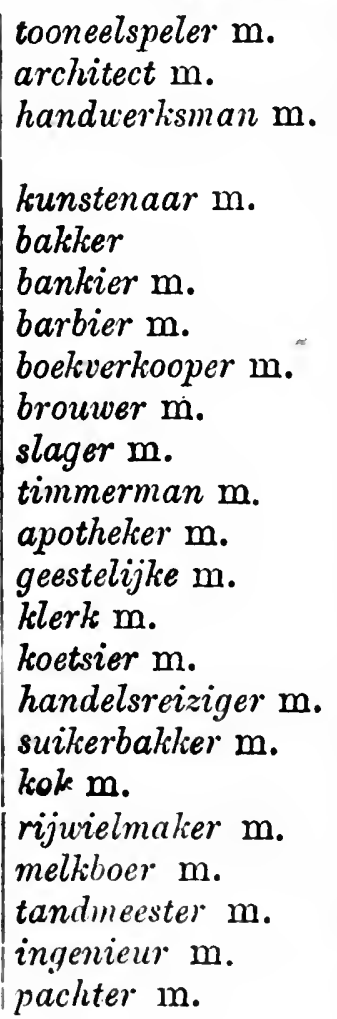 & $\begin{array}{l}\text { ha(h)ntwe(r)rks- } \\
\quad \text { ma(h)n } \\
\text { kunstenahr } \\
\text { ba(h)k'ke(r)r } \\
\text { ba(h)nkeer } \\
\text { ba(h)r'beer } \\
\text { boo'kfe(r)rkoh } \\
\text { brouwe(r)r [pe(r) } \\
\text { slahge(r)r } \\
\text { tim'me(r)rma(h)n } \\
\text { ahpohtayke(r)r } \\
\text { gaysste(r)le(r)ke(r) }\end{array}$ \\
\hline
\end{tabular}




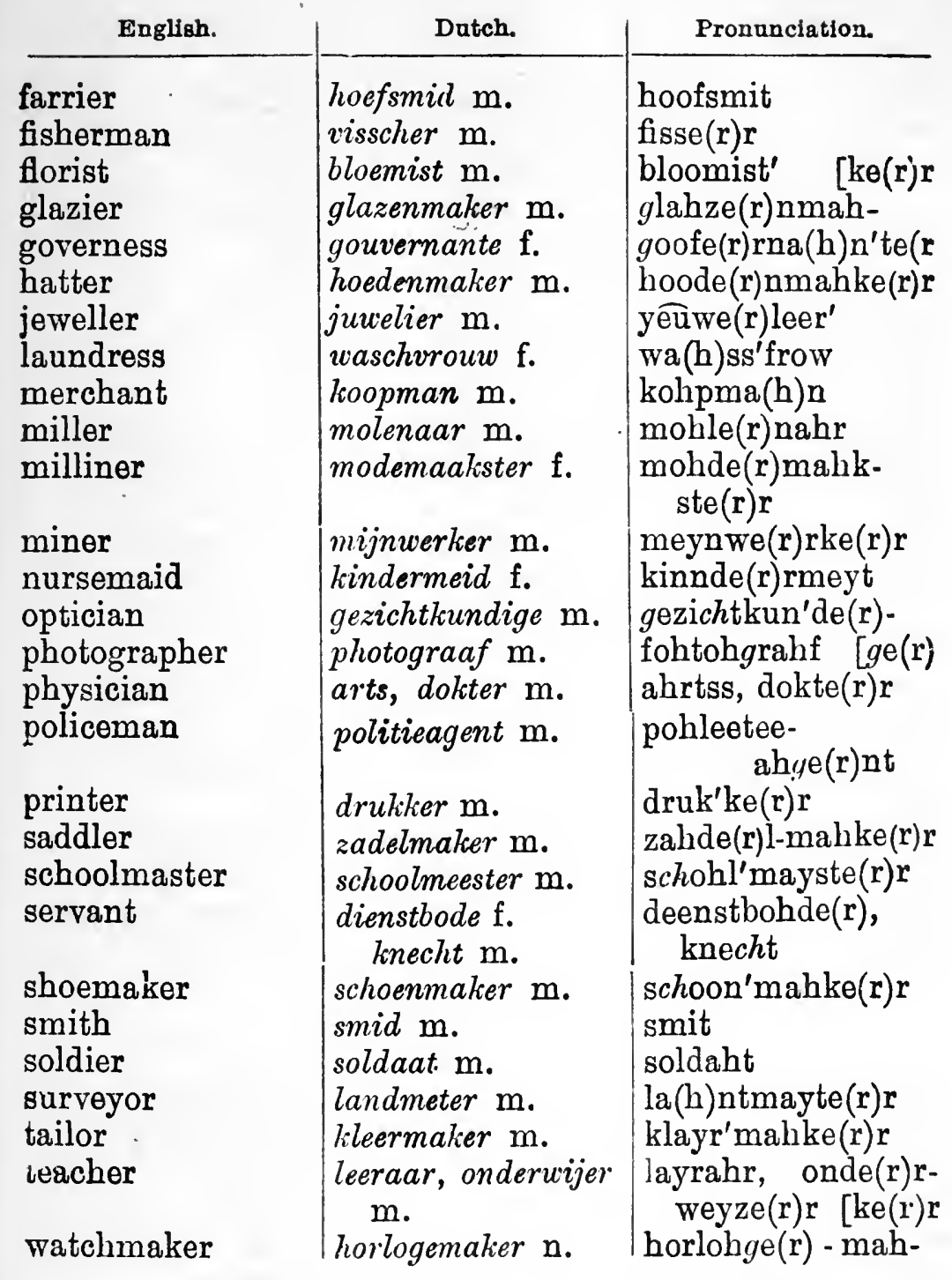

21. Ships and Shipping. (Schepen en Verscheping.) (For Steamboat, see page 99.)

\begin{tabular}{l|l|l} 
Anchor & lanker $\mathrm{n}$. & $\mathrm{a}(\mathrm{h}) \mathrm{nke}(\mathrm{r}) \mathrm{r}$ \\
boat & $\begin{array}{l}\text { boot } \mathrm{f} . \\
\text { boeg } \mathrm{m} .\end{array}$ & boht \\
bow & booch \\
cabin & kabel $\mathrm{m} .$, hut $\mathrm{f}$. & kahyoyt, hut \\
cable & kahbe(r)l
\end{tabular}




\begin{tabular}{|c|c|c|}
\hline English. & Dutch. & Pronnnclation. \\
\hline captain & kapitein $\mathrm{m}$ & kahpeeteyn \\
\hline compass & kompas $\mathrm{n}$. & kompa(h)ss \\
\hline $\operatorname{cours} \theta$ & koers $\mathrm{m}$. & koorss \\
\hline crew & bemanning $\mathrm{f}$. & be(r)ma(h)nning \\
\hline deck & dek $\mathrm{n}$. & dek \\
\hline flag & vlag $\mathrm{f}$. & $\mathrm{fla}(\mathrm{h}) \mathrm{ch}$ \\
\hline harbour (port) & haven $\mathrm{f}$. & hahte(r)n \\
\hline helm & roer $\mathrm{n}$. & roor \\
\hline kee] & kiel f. & keel \\
\hline landing-stage & landingplaats $\mathrm{f}$. & la(h)nding-plahts \\
\hline $\begin{array}{l}\text { light-house } \\
\text { mast }\end{array}$ & $\begin{array}{l}\text { vuurtoren } \mathrm{m} \text {. } \\
\text { mast } \mathrm{m} \text {. }\end{array}$ & $\begin{array}{l}\text { feùr'tohre }(r) n \\
\text { ma(h)sst }\end{array}$ \\
\hline man-of-rvar & oorlogsschip n. & ohr'logschip \\
\hline $\begin{array}{l}\text { merchantman } \\
\text { oar }\end{array}$ & $\begin{array}{l}\text { koopvaardijsclipp } \mathrm{n} \text {. } \\
\text { roeiriem m. }\end{array}$ & kohp'fahrdoyschip \\
\hline pilot & loods $\mathrm{m}$. & lohts \\
\hline rope & touw $\mathrm{n}$. & tow (like now) \\
\hline il & zeil $\mathrm{n}$. & zeyl \\
\hline seaman (sailor) & matroos $\mathrm{m}$. & ma(h)trohss. \\
\hline steers & $\begin{array}{l}\text { schip } 1 . \\
\text { stuurman m. }\end{array}$ & steurma (h)n \\
\hline stern & achtersteven & $\mathrm{a}(\mathrm{h}) \operatorname{chte}(\mathrm{r}) \mathrm{rst}$ \\
\hline $\begin{array}{l}\text { tug } \\
\text { wharf }\end{array}$ & $\begin{array}{l}\text { sleepboot f. } \\
\text { werf, kaai f. }\end{array}$ & $\begin{array}{l}\text { slayp'boht }[\mathrm{f} \\
\text { werrf, kah'i }\end{array}$ \\
\hline
\end{tabular}

\section{Trayelling. (Reizen.)}

(For Conversations see pages 97, 99. 100.)

\begin{tabular}{|c|c|c|}
\hline $\begin{array}{l}\text { Arrival } \\
\text { bill } \\
\text { cabman } \\
\text { carriage } \\
\text { coach } \\
\text { coachman (driver) } \\
\text { departure } \\
\text { engine } \\
\text { fare } \\
\text { guard } \\
\text { guide } \\
\text { guide-book } \\
\text { bat-box }\end{array}$ & $\begin{array}{l}\text { aankomst } \mathrm{f} . \\
\text { rekening } \mathrm{f} . \\
\text { koetsier } \mathrm{m} . \\
\text { rijtuig } \mathrm{n} . \\
\text { koets } \mathrm{f} . \\
\text { koetsier } \mathrm{m} . \\
\text { vertrek } \mathrm{n} . \\
\text { locomotief } \mathrm{f} . \\
\text { vracht } \mathrm{f} . \\
\text { conducteur } \mathrm{m} . \\
\text { gids } \mathrm{m} . \\
\text { reisgids } \mathrm{n} . \\
\text { hoedendoos } \mathrm{f} .\end{array}$ & $\begin{array}{l}\text { ahn'komsst } \\
\text { rayke(r)ning } \\
\text { kootseer' } \\
\text { reytoych } \\
\text { koots } \\
\text { kootseer' } \\
\text { fe(r)rtrek } \\
\text { lohkohmohteef' } \\
\text { fra(h)cht } \\
\text { konduktu(r)r' } \\
\text { gidss } \\
\text { reyss'gidss } \\
\text { hoode(r)ndohss }\end{array}$ \\
\hline
\end{tabular}




\begin{tabular}{|c|c|c|}
\hline & Dut & ion. \\
\hline & & oh \\
\hline & & \\
\hline & ines $\mathrm{f}$. & ka(h)ste(r)ley \\
\hline f hotel) & kastelein & ka(h)stele \\
\hline ge & bag & \\
\hline & $k$ & $\mathrm{ka}(\mathrm{l}$ \\
\hline us & 0 & orm' \\
\hline at & $b$ & De \\
\hline & & \\
\hline & & sp \\
\hline ge & wagg & wah'go(h)n [see-on' \\
\hline & ion $\mathrm{n}$. & spohr'wech-st \\
\hline & 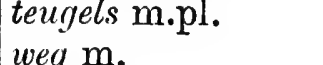 & ) lss \\
\hline & zadel & $z^{\prime} a h^{\prime} d$ \\
\hline el* & stationschef & 'shef \\
\hline $\begin{array}{l}\text { steamer } \\
\text { ticket }\end{array}$ & $\begin{array}{l}\text { stoomboot. } \mathrm{f} . \\
\text { biljet, kaartje } \mathrm{n}\end{array}$ & $\begin{array}{l}\text { stohm'boht } \\
\text { bilyet', kahrtye }(\mathrm{r})\end{array}$ \\
\hline- , first-c & eer & $\begin{array}{l}\text { ayrste }(\mathrm{r}) \mathrm{kla}(\mathrm{h}) \mathrm{s}- \\
\text { se(r) bilyet' }\end{array}$ \\
\hline - -offic & loket $\mathrm{n}$. & lohkett \\
\hline -, return & urbiljet & oor'bilyet \\
\hline $\operatorname{tr}$ & $r$ & $\operatorname{re}(\mathrm{r}) \mathrm{n}$ \\
\hline & & $e(r) r$ \\
\hline $\mathrm{om}$ & wachtkamer $\mathrm{f}$. & $\begin{array}{l}\text { reyss } \\
\text { wa(h)chtkahme(r }\end{array}$ \\
\hline
\end{tabular}

23. Countries and Nations. (Landen en Volken.)

Africa

America

American

Asia

Australia

Austria

Belgian

Belgium

Canada

Cape, the

China
Afrika n.

Amerika n.

Amerikaan m.

Azië n.

Australië n.

Oostenrijk n.

Belg m.

België n.

Canada

de Kaap f.

China $\mathrm{n}$. a(h)f'reekah

ahmay'reekah

ahmayreekahn'

ah'zee-e(r)

ohstrahlee-e(r)

oh'ste(r)nreyk

belch

bel'gee-e(r)

kahnahdah

de(r) kahp

sheenah 


\begin{tabular}{|c|c|c|}
\hline Engl & Dutch. & Pronunciation. \\
\hline$u$ & land $\mathrm{n}$. & $\mathrm{la}(\mathrm{h}$ \\
\hline$u$ & Cuba n. & $\mathrm{ab}$ \\
\hline Dane & Deen m. & dayn \\
\hline Der & Denemarken n. & dayne(r)ma(h)rken \\
\hline $\operatorname{man}$ & Hollander $\mathrm{m}$. & hol'la(h)n-de(r)r \\
\hline & rijk n. & reyk \\
\hline hman & Engeland $\mathrm{n}$. & eng'e(r)la(h)nt \\
\hline $\begin{array}{l}\text { Englishman } \\
\text { Europe }\end{array}$ & $\begin{array}{l}\text { Engelschman m. } \\
\text { Europa n. }\end{array}$ & $\begin{array}{l}\text { eng'e(r)Issma (h)n } \\
\mathrm{u}(\mathrm{r}) \mathrm{rohpah}\end{array}$ \\
\hline Fra & Frankrijk n. & frahnk'reyk \\
\hline Fre & Franschman $\mathrm{m}$. & fra(h)nssma(h)n \\
\hline $\mathrm{Ge}$ & scher $n$. & doytsse $(r) r$ \\
\hline & Duitschland $\mathrm{n}$. & doytssla $(\mathrm{h}) \mathrm{nt}$ \\
\hline Ho & Holland $\mathrm{n}$. & hol'la(h)nt \\
\hline $\mathrm{Hu}$ & gaar $\mathrm{m}$. & hongal \\
\hline & Hongarije $\mathrm{n}$. & hongahrey'e(r) \\
\hline In & $\ddot{e} \mathrm{n}$. & inde \\
\hline Inc & $t-I n$ & ohst indee $-\theta(r)$ \\
\hline,$- W$ & $\begin{array}{l}n \\
t\end{array}$ & west indee-e(r) \\
\hline and & $\begin{array}{l}\text { Ier } \\
\text { Ier }\end{array}$ & eer la \\
\hline & Italiaan $\mathrm{m}$. & eetahleeahn \\
\hline to & Italië $\mathrm{n}$. & eetahle \\
\hline Ja1 & Japan $\mathrm{n}$. & yahpal \\
\hline & $\begin{array}{l}\text { koninkrijk } \mathrm{n} . \\
\text { Nieuw Zeland }\end{array}$ & $\begin{array}{l}\text { kohnink } \\
\text { neew-za }\end{array}$ \\
\hline Nor & Noorwegen $\mathrm{n}$. & nohrwayge(r)n \\
\hline Nor & Noor m. & nohr \\
\hline & Rusland n. & russ'la(h)nt \\
\hline & $\begin{array}{l}\text { Rus m. } \\
\text { Schot m. }\end{array}$ & $\begin{array}{l}\text { russ } \\
\text { schot }\end{array}$ \\
\hline Scotland & Schotland n. & schot'la(h)nt \\
\hline Spain & Spanje m. & spa(h)nye(r) \\
\hline Spaniard & Spanjaard $\mathrm{m}$. & spa(h)n'yahrt \\
\hline $\begin{array}{l}\text { Swede } \\
\text { Sweden }\end{array}$ & Zweden n. & $\begin{array}{l}\text { zwayt } \\
\text { zwayde(r)n }\end{array}$ \\
\hline Swiss & Zwitser $\mathrm{m}$. & zwit'se(r) $r$ \\
\hline Switzerland & Zuitserland $\mathrm{n}$. & zwit'se(r)rla(ll)nt \\
\hline & $\mathrm{m}$ & turk \\
\hline Turkey & Turkije n. & I turkaye(r) \\
\hline
\end{tabular}




\section{Commercial and Trading Terms. \\ (Handels- en Koopmanstermen.) \\ (For Conversations see page 117.)}

English.

Dutch.

Pronunciation.

Account, to settle een rekening vereffe- ayn rayke $(\mathrm{r})$ ning

an

- to close

-, current

- , joint

- of sale

acknowledgment advance (of money) advice, letter of agency

agent

apprentice

arrangement

arrear, in

assets

authorize, to

average (to ships)

balance

- of account

- -sheet

banking

bearer

bill, accommoda-

- at sight

- at three months' date

- of lading

bond, in

bonded goods

book-keeper

brokerage

business, wholesale 一, retail

buyer

\section{nen}

rekening afsluiten

- courant

gemeenschappelijke

verkooprekening ontvangstbericht $\mathrm{n}$. voorschot $\mathrm{n}$. adviesbrief $\mathrm{m}$. agentschap $\mathbf{n}$. agent $\mathrm{m}$. leerling $\mathrm{m}$. schikking $\mathrm{f}$. ten achteren actief $\mathrm{n}$. machtigen averij $\mathrm{f}$. saldo $\mathrm{n}$. saldo rekening balans $\mathrm{f}$. bankwezen $\mathrm{n}$.

toonder $\mathrm{m}$.

formawissel

wissel op zicht

- drie maanden na zicht

vrachthrief

in entrepôt

opgeslagen goederen

boekhouder $\mathrm{m}$. makelarij $\mathrm{f}$. groothandel m. kileinhandel $\mathrm{m}$. kooper $\mathrm{m}$. vrachtvrij fe(r)reffe $(r) n e(r) n$ - $\mathrm{a}(\mathrm{h})$ fsloyte $(\mathrm{r}) \mathrm{n}$ - -koora(h)nt ge(r)maynscha(h)p. pe(r)le(r)ke(r) [ning ferkohprayke(r)ontfa(h)ngstber. fohischot [richt $a(h) d v e e s s b r e e f$ $\mathrm{a}(\mathrm{h})$ gentscha(h)p a(h)gent layrling schikking ten $\mathrm{a}(\mathrm{h}) c h$ tere $(\mathrm{r}) \mathrm{n}$ a(h)kteef ma(h)chte(r)gen ahfe(r)rey sa(h)ldoh sa(h)ldoh rayke(r). bahla(h)nss ba(h)nkwayze(r)n tohnde(r)r formahwisse $(r) 1$ wis'se(r)l op zicht -dree mahnde(r)n nah zicht fra(h)chtbreef in $a(h) n t r e(r) p o h$ op-ge(r)slahge(r)n goo'dere $(r) n$ boo'khowde(r)r mahke(r)lahrey groht'ha(h)nde(r)l kleyn'ha(h)nde(r)l kohpe (r)r fra(h)chtfrey 


English.
cartage
catalogue
charter a ship, to
claim, to send
in a

-, to make good a clearing-house clerk company, limited -, joint-stock

\section{complaint}

contract

cost, insurance and

freight (c.i.f.)

credit balance creditor

custom-house

- officer

customs, duties damages

—, to claim

dated at three months'

debit and credit

debtor

deliver, to

delivered free

demurrage(days of)

director

discount

- , to

dividend warrant

dock and barbour

double entry [dues
Dutch.

vracht

catalogus $\mathrm{m}$.

een schip huren

een eisch instellen

zijn recht staven

rescontrehuis $\mathrm{n}$.

klerk $\mathrm{m}$.

maatschappij $\mathrm{f}$.

maatschappij

aandeelen

klacht $\mathrm{f}$.

contract $\mathrm{n}$.

kosten m.pl., verzekering f., en vracht $\mathrm{f}$.

credit-saldo $\mathrm{n}$.

crediteur $\mathrm{m}$.

douanen-kantoor $\mathrm{n}$.

douanier m., kommies $\mathrm{m}$.

rechten $\mathrm{n} . \mathrm{pl}$.

vergoeding $\mathrm{f}$.

schadevergoeding

vorderen

drie maanden na dato

debet $\mathrm{n}$., en credit $\mathrm{n}$. debiteur $\mathrm{m}$.

leveren

franco levering $\mathrm{f}$.

ligdagen m.pl. commissaris $\mathrm{m}$.

korting $\mathrm{f}$.

disconteeren

coupon $\mathrm{f}$.

dokgeid $\mathrm{n}$.

dubbel boekhou-

den $\mathrm{n}$.
Pronunciation.

fra(h)cht

kahtahloh'guss

ayn schip hêre(r)n

ayn eyss instel-

$\operatorname{le}(\mathrm{r}) \mathrm{n}$

$[\mathrm{fe}(\mathrm{r}) \mathbf{n}$

zeyn recht stah-

re(r)skontre(r)-

kle(r)rk

mahtscha(h)ppey

mahtschia(h)ppey

op ahndayle(r)n

kla(h)cht

kontra(h)kt

koste $(r) n, \quad f e(r) r-$

zaykering,

fra (h) cht $\mathrm{f}$.

kraydit'-sa(h)ldoh

kray'deetu(r)r

dooahne(r)nka(h)n.tohr

dooahniay, kommeess

rechte(r)n

fe(r)rgoo'ding

schahde(r)fergoo'-

ding fordere $(r) n$

dree mahnde(r)n

nah dah'tol.

daybe(r)t en kray-

daybeetu(r)r [dit.

layfere $(r) n$

fra(h) nkohlayfe(r)-

ligdahge(r)n [ring commissahrriss

korting

disko(h)ntayre(r)n

koopo(h)n

dok'gelt

dubbe(r)l boo'k-

howde(r)n 


\begin{tabular}{l}
\multicolumn{1}{c}{ English. } \\
\hline drawer \\
duty, export \\
- -free \\
-, import \\
-, liable to \\
enclosed \\
enclosure \\
endorse, to \\
endorsement \\
exchange (the) \\
firm \\
forwarding \\
- agent
\end{tabular}

free on board (f.o.b.) gross weight [tor guarantee, guaranI.O.U. import and export information inquire, to inquiry insurance, marine - broker

- company

- policy

- premium

insure against, to

interest, rate of to introduce introduction landing charges

liabilities

lighterage load, to loss manager

trekker $\mathrm{m}$. uitvoerrecht tolvrij invoerrecht onderherig aan tol ingesloten bijlage $\mathrm{f}$. endosseeren endossement $\mathbf{n}$. beurs $\mathrm{f}$. firma $\mathrm{f}$. verzending $\mathrm{f}$. expediteur $\mathrm{m}$. vrij aan boord bruto-gewicht $\mathrm{n}$. borg $\mathrm{m}$. ik ben u schuldig invoer en uitvoer informatie $\mathbf{f}$. vragen vraag $\mathrm{f}$. zeeverzekering assurantie-makelaar

\section{$\mathrm{m}$.}

verzekering-maatschappij $\mathrm{f}$.

\section{- -polis}

verzekering-premie verzekeren tegen

de rentevoet $\mathrm{m}$. invoeren

introductie $\mathrm{f}$. onkosten van lossen verplichtingen f.pl. lichtergeld $\mathrm{n}$. laden verlies $\mathrm{n}$. directeur $\mathrm{m}$.
Pronuuciation.

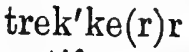
oyt'foorrecht tolfrey in'foorrecht [ahn tol onde(r)rliayfe(r)ch inge $(r)$ slohte $(r) n$ beylahge(r) endossayre $(r) n$ a(h)ndosse(r)ment bu(r)rss firmah fe(r)rzending expaydeetu(r)r frey ahn bohrt

brêtoh-ge(r)wicht bohrch [de(r)ch ik ben $\overparen{\text { eu }}$ schulinfoor en oyt'foor informahsee frahge $(r) n$ frahch

zayferzaykering a(h)ssêra(h)nteemahke(r)lahr ferzaykering-mahtscha(h)ppey

- pohliss [mee ferzaykering - prayferzaykere(r)n tay$g e(r) n$ $\operatorname{de}(\mathbf{r})$ ren'te(r)tuo-t infoore $(r) r$. introhduksee onkoste(r)n fa(h)n losse(r)n

$\mathrm{fe}(\mathrm{r}) \mathrm{rplichting-e(r)n}$ lichtergelt lahde $(r) n$ fe(r)rleess $\operatorname{deerektu(r)r}$ 


English.
market
- , cattle
- day
- dues
-, foreign
-, home
-, London
-, meat
- price
negotiable, saleable
offer (for sale), to
offers
order, to
packing
partnership
payable at sight
port of delivery
price, cost-
- -list
-, selling
-, wholesale

quotation (of price) -, lowest

receipt

reduction

register, to

registered letter

- tonnage

rent

representative

salesman (seller)

settling days

shares, debenture

-, ordinary

-, preference

markt $\mathrm{f}$.

veemarkt

marktdag $\mathrm{m}$.

- gelden

buitenlandsche

markt [bruili $\mathrm{n}$.

binnenlandsch ver.

de Londensche

markt

vleeschhal

marktprijs $\mathrm{f}$.

gangbaar.

te koop bieden

aanbiedingen n.pl.

bestellen

emballage $\mathrm{f}$.

i'ennootschaap $\mathrm{f}$.

betaalbaar op zicht

losplaats $\mathrm{f}$.

inkoopsprijs $\mathrm{m}$.

prijslijst $\mathrm{f}$.

verkoopsmijs

en gros prijs

prijsnoteering $\mathrm{f}$.

uiterste prijs

ontvangst $\mathrm{f}$.

vermindering $\mathrm{f}$.

registreeren

aangeteekende brief $\mathrm{m}$.

tonneninhoud

huur, pacht $\mathrm{f}$.

representant $\mathrm{m}$.

verkooper $\mathrm{m}$.

rescontre-dagen

obliqatiën

gewone aandeelen

preferente aandeelen
Pronunciation.

ma(h)rkt

fayma(h)rkt

ma(h)rkt-dahch

_- -gelde(r)n

boytenla(h)ndsse(r)

ma(h)rkt [fe(r)rbroyk

binne(r)nla(h)nts

$\operatorname{de}(r)$ londe(r)nse(r) ma(h)rkt

flayss-ha.(h)l

ma(h)rkt-preyss

ga(h)ngbahr

te(r) kohp beede $(r) n$

ahnbeeding - $e(r) n$

beír)stellen [she(r)

a(h)mba(h)llah-

fennohtschahp

be(r)tahlbahr op

lossplahts [zicht

inliohps'preyss

preyssleysst

fe(r)rkohpspreyss

a(b)n groh preyss

preyssnoh'tayring

oyterste(r) preyss

ontfa(h)ngst

fe(r)rmin'dering rer $(g)$ istrayre $(r) n$ ahnge( $r$ )tayken-

$\operatorname{de}(r)$ breef tonne(r)ninhowt hêur, pa(h)cht repraysenta(h)nt fe(r)rkolıpe(r)r [gen re(r) skontre(r)-dahobbleegahtsee-e(r)n ge(r)wohne(r) ahndaylen

prayfayrrente(r)

ahndaylen 


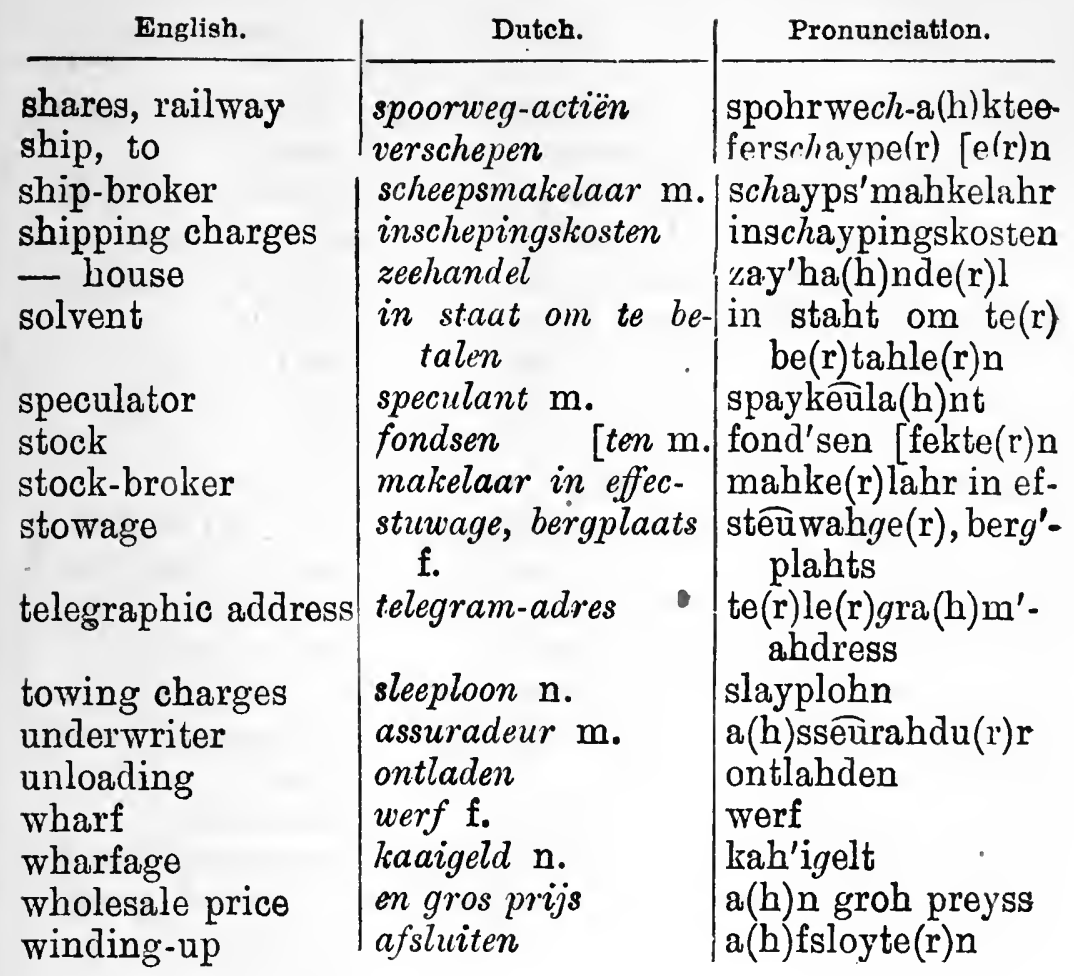

25. Cycling. (Wielrijden.)

(For Conversations see page 113.)

Axle

ball bearings

bell

bicycle

"bike"

brake

cement

chain

chainless

chain-wheel

$\operatorname{con} \theta$

connection

crank

cyclist

cyclist, lady

as $\mathrm{f}$.
kogeldragers m.pl.
bel f.
rijwiel, tweewie-
$\quad$ ler $\mathrm{m}$.
fiets $\mathrm{f}$
rem $\mathrm{f}$.
cement $\mathrm{n}$.
ketting $\mathrm{m}$.
acatène
kettingrad $\mathrm{n}$.
conus $\mathrm{m}$.
koppeling $\mathrm{f}$.
krulk f.
wielrijder $\mathrm{m}$.
wielrijderes $\mathrm{f}$.

$\mathrm{a}(\mathrm{h}) \mathbf{s}$

kohgeldrahge(r)rs

bell

rey'weel, tway'weele $(\mathbf{r}) \mathbf{r}$

feets

rem

se(r)ment

ketting

ahkataine

ketting-ra(h)t

kohnuss

koppe(r)ling

kruk

weel'reyde(r)r

weelreyderess ${ }^{\prime}$ 


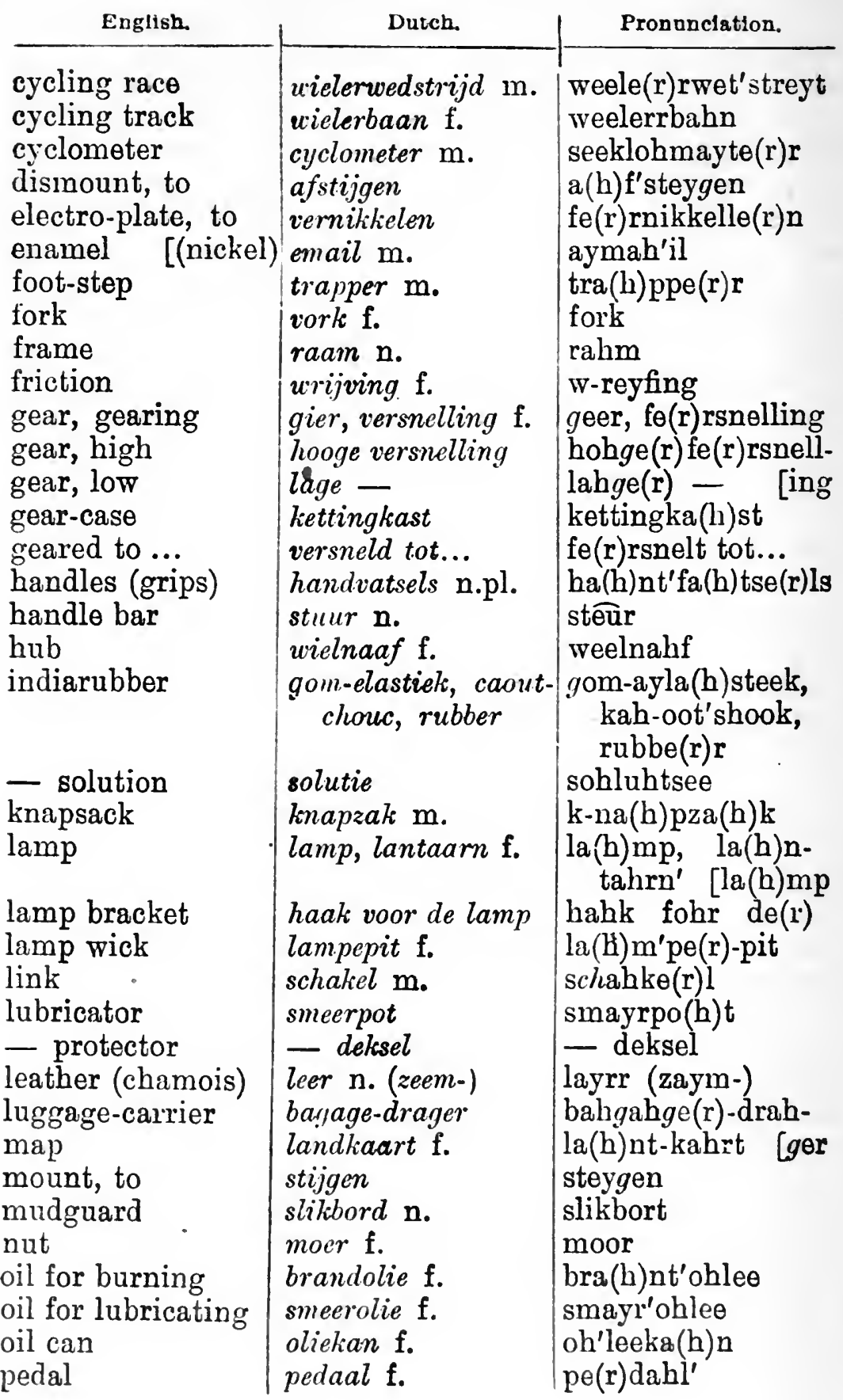


English.

Dutch.

Pronunciation.

pedal rubber

pump

pump tube

pump, to

rags, some old

removable

repairing outfit

rim

saddle

saddle bag

saddle pillar

screw

screw-bolt of chain

screwdriver

screw, to

spanner

spokes

spring

start off, to

steer, to

strap (leather)

take to pieces, to

tighten up, to

toe clip

tricycle

srousers' clip

tube, inner

tube (outer cover)

tyre (pneumatic)

unscrew, to

valve

wallet

wheel (front)

- (back)

pedaal-caoulchouc
pomp f.
pompslang $\mathrm{f}$.
pompen
eenige oude lompen,

lappen

verplaatsbaar

reparatiedons $\mathrm{f}$.

velg $\mathrm{f}$.

zadel $\mathrm{m}$.

zadeltasch $\mathrm{f}$.

zadelpen

schroef $\mathrm{f}$.

kettingboutje

schroevendraaier $m$.

aanschroeven

spakenspanner $\mathrm{m}$.

spaken f.pl.

veer $\mathrm{f}$.

vertrekken

sturen

(lederen) riem $\mathrm{m}$.

uit elkander nemen

spannen

teenhaak

driewieler $\mathrm{m}$.

broekveer $\mathrm{f}$.

binnenband $\mathrm{f}$.

buitenband

band (pneumatische)

losschroeven

ventiel $\mathrm{n}$.

zak $\mathrm{m}$.

voorwiel $\mathrm{n}$.

achterwiel pe(r)dahl'-kah-oot'pomp [shook

pomp-sla(h)ng pompe(r)n

ayne(r)ge(r) ow-

$\operatorname{de}(r)$ lompe(r)n

la(h)ppen

fe(r)plahtssbahr

raypahrahtsee-

fellch

zahde(r)l

[dohss

zahde(r)l-ta(h)ss'

zahde(r)l-penn

schroof

ket'ting-bowtye(r)

schroof'e(r)ndrah'i-

$$
e(r) r
$$

ahn'schroofe(r)n spahkenspa(b)n-

spahken [ne(r)r

fayr

fe(r)rtrekke(r)n

steuren

(layderen) reem

oyt elka(h)nder

nayme $(r) n$

spa(h)nnen

taynhahk

dree'weeler

broo'k-fayr

bin'nen-ba(h)nt

boy'te(r)nba(h)nt

band [pneumahtisse(r)]

los'schroofe(r)n

fenteel

$\mathrm{za}(\mathrm{h}) \mathrm{k}$

fohrweel

$\mathrm{a}(\mathrm{h})$ chterweel 
26. Motoring Terms. (Automobiel benamingen.)

(See also Crchivo, pp. 45 \& 113, and Notorixg, p. 115.)

\begin{tabular}{|c|c|c|}
\hline & & \\
\hline $\begin{array}{l}\text { bler) } \\
\text { pinion } \\
1 \\
\text { ty } \\
\text { - box } \\
\text { cer) } \\
\text {-valve } \\
1\end{array}$ & $\begin{array}{l}\mathrm{n} . \quad \text { (voor- } \\
\text { beweging } \\
n \text { ) } \\
\text { acht } \mathrm{f} . \\
g \mathrm{f} . \\
\text { (handvat) } \\
\mathrm{m} . \quad \text { [m. } \\
\text { f. (wat) } \\
\mathrm{n} . \\
\text { artsche ver- } \\
g \mathrm{f} . \\
\text { rt f. } \\
\text { aart } \\
\text { el m. } \\
\text { ersnelling } \\
\text { lg } \mathrm{f} .\end{array}$ & $\begin{array}{l}\text { a(h)klêum } \text { eulah'- } \\
\text { ba(h)tterey' [tor } \\
\text { remba(h)nt (stah- } \\
\text { ka(h)m [le(r)n } \\
\text { ka(h)rbeurah'tor } \\
\text { spa(h)nnen fa(h)n } \\
\text { de(r) kettinge(r)n } \\
\text { ta(h)ng, fork } \\
\text { konta(h)ktbray- } \\
\text { ke(r)r, tril'le(r)r } \\
\text { shohfurr } \\
\text { dreyfka(h)mweel } \\
\text { elbohch }\end{array}$ \\
\hline
\end{tabular}


27. Photography. (Photografie.)

(For Conversation, soe p. 116.)

\begin{tabular}{l} 
English. \\
\hline Backgrounds \\
backing paper \\
camera \\
dark slide (doubl \\
dishes for develop- \\
ing \\
- for fixing \\
draining rack \\
dry plates \\
exposure \\
flim \\
flash lamp \\
focus \\
focussing glasses \\
funnels \\
glass measure \\
half-plate \\
iris diaphragms
\end{tabular}

lamp, dark-room

lens

mount

plate-holder

plate-lifter

platinotypes

printing

- frame

scales and weights

shutter

Dutch.

Pronunciation.

achtergronden $\mathrm{m} . \quad \mathrm{a}(\mathrm{h})$ chte $(\mathrm{r}) \mathrm{rgronden}$ - stof

photografie-papier fohtohgrahfee'pah camera f. [n. kahmerah

- stof [peer

châssis (dubbel) m. sha(h)ssee [dub-

be $(\mathrm{r}) 1]$

schalen (m.pl.) om schable(r)n om te(r)

te ontuikkelen ontwikke(r)le(r)n

fixeerschalen

droogrek $\mathrm{n}$.

droge platen

belichting $\mathrm{f}$.

film $\mathrm{n}$.

magnesium licht $\mathbf{n}$.

brandpunt $\mathrm{n}$.

instelloep

trechters m.pl.

glasmaat f.

halve plaat $\mathrm{f}$.

iris diaphragmais

feexayrschahle(r)n

drohgrek

drohge(r) plahte(r)n be(r)lichting

film

[licht

ma(h)gnayseeum

bra(h)nt-punt

instel-loop

trechte(r)rss

$g$ la(h)ssmaht

ha(h)lfe(r) plaht

coriss deeahfra(h)gmahs

lamp voor de donkeve la(h)mp fohr de(r)

kamer

lens $\mathrm{f}$.

passe partout

plaatvasthouder

plaatheffer

platinadruk

drukken $\mathrm{n}$.

kopieërraam f.

weegschalen en

gewichten

sluiter f.

- voor tijd en momentopname

persrollen van massief caoutchouc don'kere(r) kah-

lenss

pa(h)ss pa(h)rtoo

plahtfa(h)sthowder

plaht-heffe $(r) r$

plah'teenahdruk

drukken

kohpee-ayr'rahm

wayg'schahlen en

ge(r)wichten

sloyte $(\mathbf{r}) \mathbf{r}$

- fohr teyt en mohment'opnahme(r)

pers'rollen $\mathrm{fa}(\mathrm{h}) \mathrm{n}$ ma(h)sseef kah[oot'shook

Dutch Self-Taught. 


\begin{tabular}{|c|c|c|}
\hline Er:glish. & Dutch. & Pronnnciation. \\
\hline specu & snelheidswijzer & snel'heydsswe \\
\hline -fold) & drievoet (toeslaand) & dree'foo-t (to \\
\hline view-t & zosker m. & sooke(r)r [sla(h)nt \\
\hline -, square & $\rightarrow$, vierkante & -, feer'ka(h)nte(r) \\
\hline,- ol &,$- l$ & 一, $\begin{array}{r}\operatorname{Ia}(\mathrm{n}) \mathrm{n} \\
\mathrm{pe}(\mathrm{r}) \mathrm{r}\end{array}$ \\
\hline ashing & spoelbakken m.pl. & spoolba(h)kke(r)n \\
\hline
\end{tabular}

28. Fishing and Shooting. (Visschon en Schieten.)

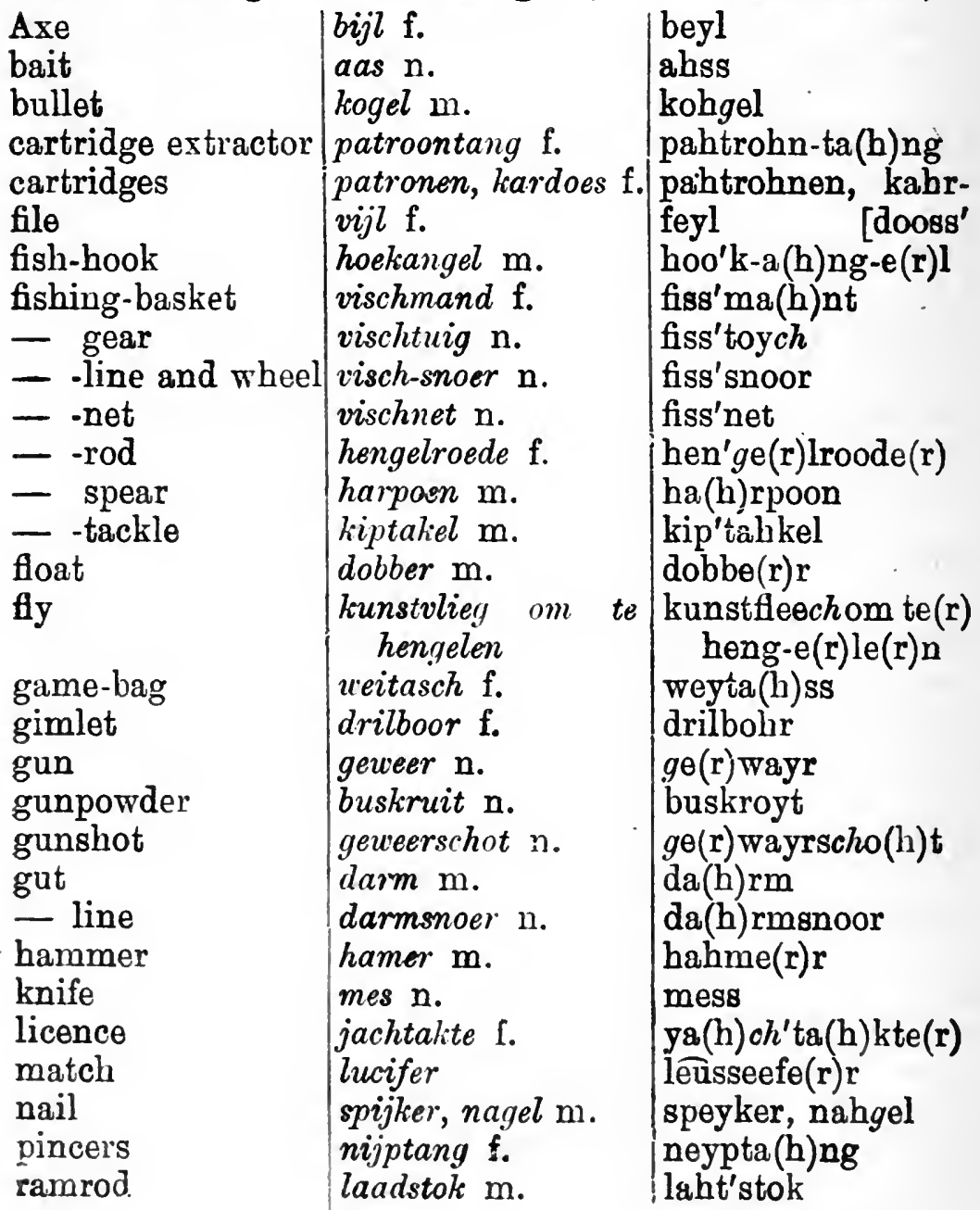




\begin{tabular}{|c|c|c|}
\hline Engltsh. & Dutch. & Pronunciation. \\
\hline $\begin{array}{l}\text { range, out of } \\
\text { rifle } \\
\text { screwdriver } \\
\text { sheath } \\
\text { shot } \\
\text { tools } \\
\text { whetstone } \\
\text { wire-nippers }\end{array}$ & $\mid \begin{array}{l}\text { buiten schot } \\
\text { geweer } \\
\text { schroevendraaier m. } \\
\text { scheede f. } \\
\text { schot } \mathrm{n} . \\
\text { gereedschap } \mathrm{n} . \\
\text { slijpsteen } \mathrm{m} . \\
\text { draadtany }\end{array}$ & $\begin{array}{l}\text { boyte(r)n scho(ta)t } \\
\text { ge(r)wayr } \\
\text { schroo'fe(r)ndrahi- } \\
\text { schayde(r) } \quad \text { e(r)r } \\
\text { schot } \\
\text { ge(r)raytsscha(h)p } \\
\text { sleyp'stayn } \\
\text { drahtta(b)ng }\end{array}$ \\
\hline
\end{tabular}

29. Amusements. (Vermalien.)

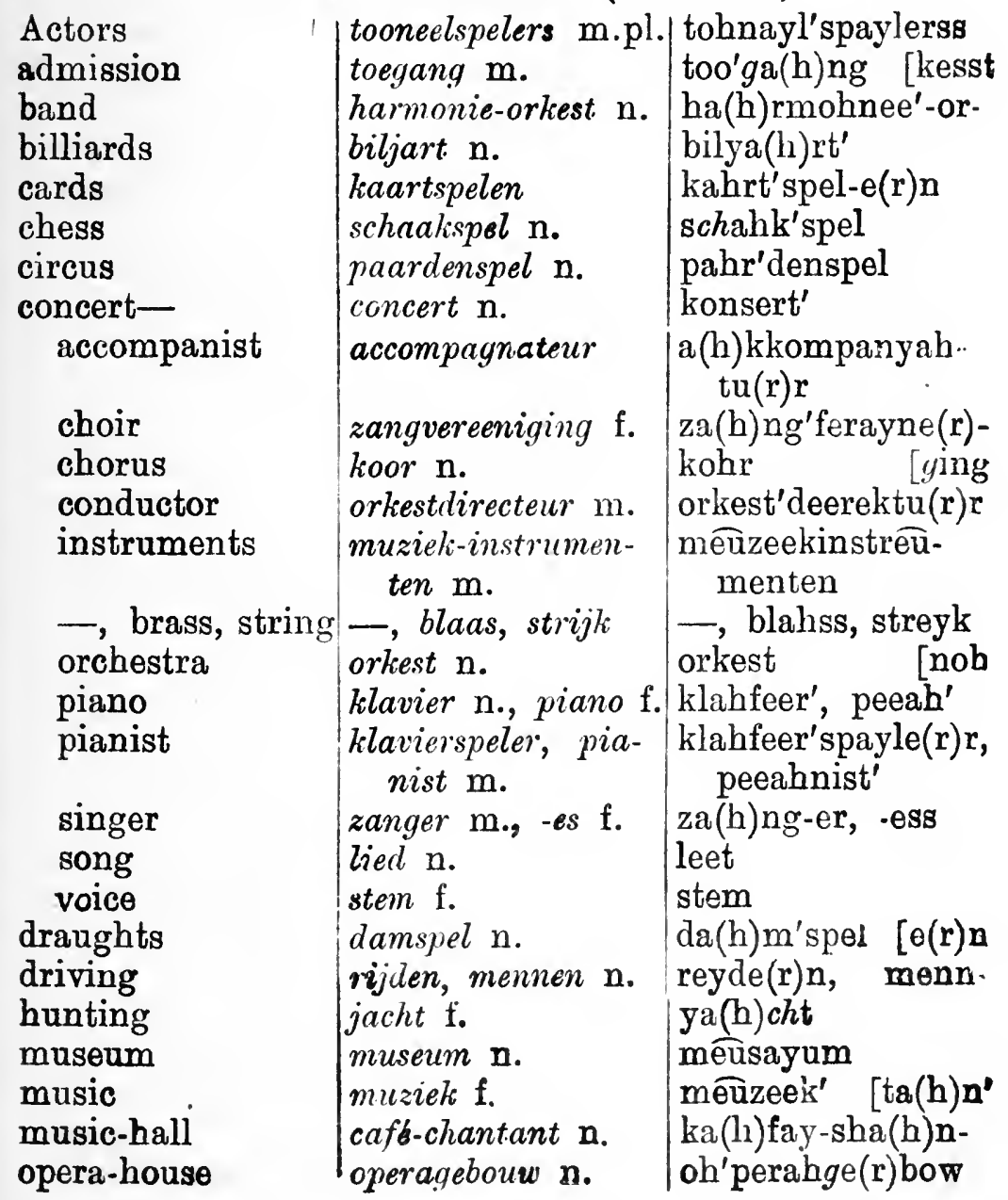




\begin{tabular}{|c|c|c|}
\hline & & \\
\hline $\begin{array}{l}\text { kates } \\
\text { kating } \\
\text { kittles } \\
\text { wimming } \\
\text { ae theatre- } \\
\text { act } \\
\text { ballet } \\
\text { box-office } \\
\text { cloak-room } \\
\text { comedian } \\
\text { comedy } \\
\text { curtain } \\
\text { dancer } \\
\text { farce } \\
\text { footlights } \\
\text { manager } \\
\text {-, stage- } \\
\text { opera-glasses } \\
\text { performance } \\
\text { play } \\
\text { programme } \\
\text { proprietor } \\
\text { refreshment- } \\
\text { scene } \\
\text { seats: } \\
\text { boxes } \\
\text { dress circlo } \\
\text { balcony } \\
\text { gallery } \\
\text { pit } \\
\text { reserved } \\
\text { stalls } \\
\text { stage } \\
\text { tickets } \\
\text { tragedy } \\
\text { tragedian } \\
\text { inlin }\end{array}$ & 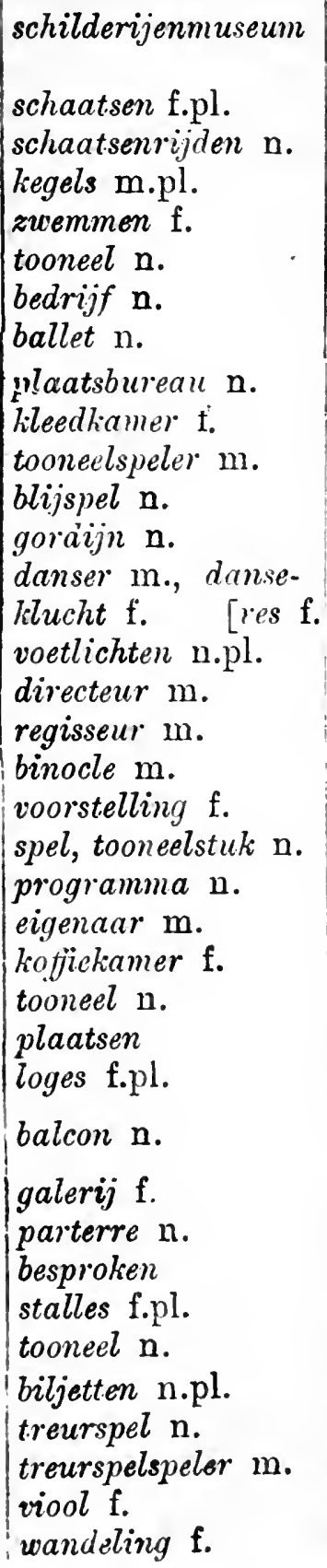 & $\begin{array}{l}\text { schilderey'en } \\
\text { mêsay'um } \\
\text { s'hahtse(r)n } \\
\text { schahtse(r)nrey- } \\
\text { liayge(r)lss [de(r)n } \\
\text { zwemme(r)n } \\
\text { tohnayl' } \\
\text { be(r)dreyf } \\
\text { ba(h)llet' } \\
\text { plahtsbeuroh } \\
\text { irlayt'kahmer } \\
\text { tohnayl'spayler } \\
\text { bleyspeyl } \\
\text { gohrdeyn' } \\
\text { da(h)n'ser, da(h)ns- } \\
\text { klucht } \\
\text { foo'tlichten } \\
\text { deerektu(r)r } \\
\text { raysheessu(r)r } \\
\text { beenok'l } \\
\text { fohr'stelling } \\
\text { spel, tohnayl'stuk } \\
\text { prohgra(h)m'mah } \\
\text { ey'ge(r)nahr } \\
\text { koffeekahmer } \\
\text { tohnayl' } \\
\text { plahtsen } \\
\text { loshe(r)ss } \\
\text { ba(h)lko(h)n } \\
\text { gahlerey' } \\
\text { pa(h)rter're(r) } \\
\text { besprohke(r)n } \\
\text { sta(h)l'less } \\
\text { tohnayl' } \\
\text { bilyetten } \\
\text { tru(r)rspel } \\
\text { trur(r)rspelsspay- } \\
\text { fohl } \\
\text { wa(b)nde(r)ling }\end{array}$ \\
\hline
\end{tabular}


30. Correspondence. (Briefwisseling.)

(For Conversations, see p. 102.)

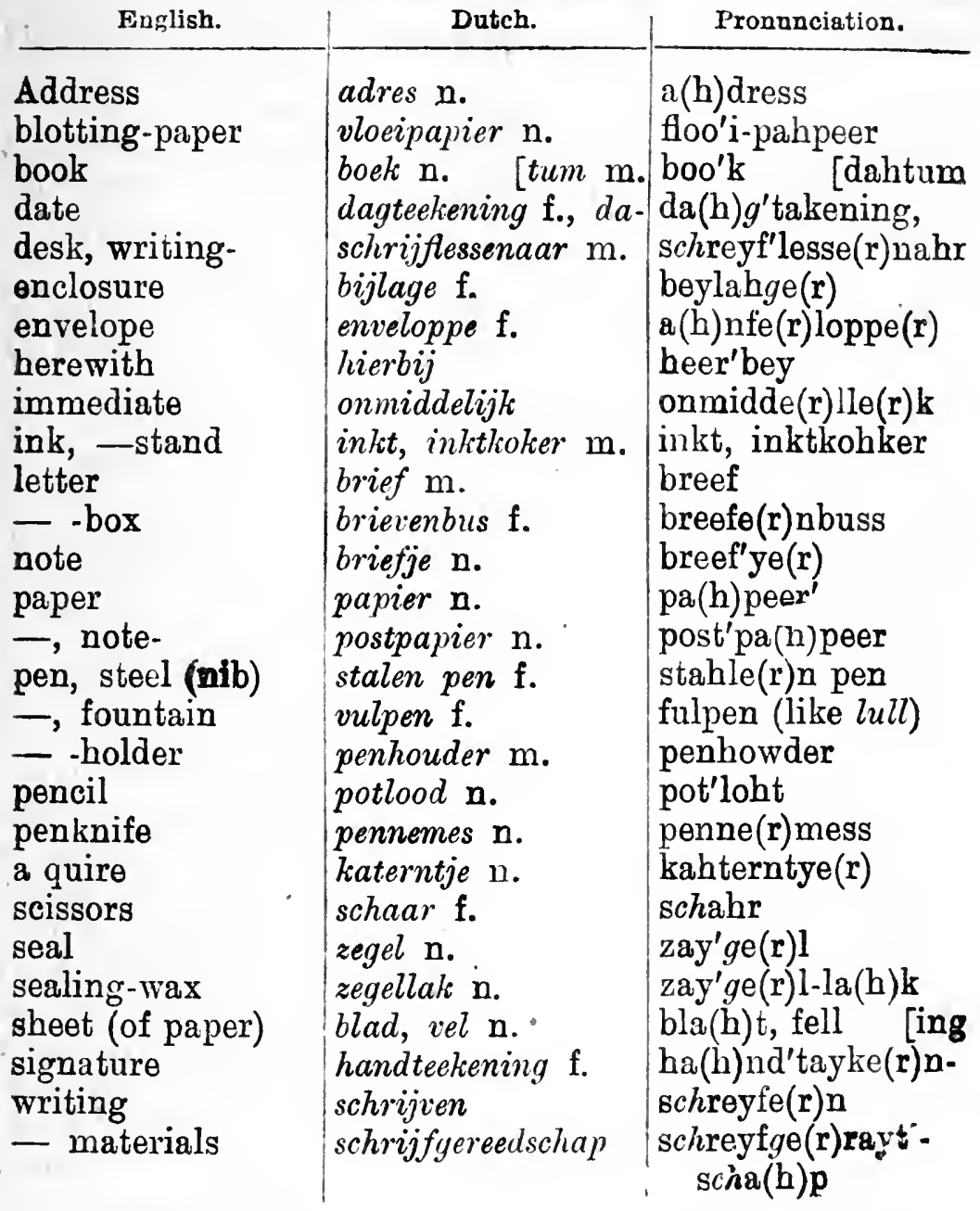

31. Post-office and Telegrams

(Post- en Telegraafkantior.)

(For Conversations, see p. 103.)

Book post counterfoil description despatch-note destination drukwerk $\mathbf{n}$. souche m. besohrijving f. depeche $\mathrm{m}$. bestemming $t$.

äru's'werk
goossje(r)
be(r)schreyfing
daypayche(r)
be(r)attemming




\begin{tabular}{l} 
English. \\
\hline excess postage \\
insured value \\
letter post \\
money order
\end{tabular}

nature of contents newspaper wrapper

\section{number}

parcel post

postage paid

- stamp

postal (pillar-) box post-card

poste-restante

postman

postmaster

post-office

re-address, to

register, to

roply paid

telegraph form

- messenger

- office

value of contents weight

-, over, under

Dutch.
strafport $\mathrm{n}$.
verzekerde waarde
brieven-post
postroissel $\mathrm{m}$.
aard van den inhoud
couranten strook $\mathrm{f}$.

getal, aantal $\mathrm{n}$. postpaketten

franco

postzegel $\mathrm{m}$.

brievenbus $\mathrm{f}$.

briefkaart $\mathrm{f}$.

poste-restante

brievenbesteller $\mathrm{m}$.

postdirecteur $\mathrm{m}$.

postkantoor $\mathrm{n}$.

op nieuw adrosseeren

aanteekenen

antwoord betaald

telegramformulier $\mathbf{f}$. telegraaf bods $\mathrm{m}$.

- kantoor

waarde van den ingewicht. $\mathbf{n}$. [houd overwicht, beneden hot gewicht
Pronanciation.

stra(h)fport

fe(r)rzayke(r)rde(r) wahrde(r)

breefe(r)n-post

post'wisse(r)l [howt

ahrt fa(h)n den in'-

koora(h)nte(r)n

strohk

$g e(\mathbf{r}) \mathrm{ta}(\mathrm{h}) \mathrm{l}, \mathrm{ahnta}(\mathrm{h}) \mathbf{l}$

post' pa(b)kette(r)n

fra(h)nkoh

post'zayge(r)l

breefe(r)nbuss

breefkahrt

post resta(h)nte(r)

breofenbesteller

post'deerektu(r)r

post'ka(h)ntohr

op nee'w a.h)dres.

say're(r)n

ahntayke(r)ne(r)n

$a(h) n t^{\prime}$ wohrt be(r)-

tahlt' [mềleer

te(r)le(r)gra(h)mfor-

te(r)le(r)grahf boh- ka(h)ntohr' [de(r) wahrde (r) fa(h)n den ge(r)wicht [in'howt oh'fe(r)rwicht, be(r)nayden het ge(r)[wicht

32. Cardinal Numbers. (Hoofdtelwoorden.)

(For Grammatical Notes see p. 81.)

Datch. Pronanciation.

1 een

2 twee

3 drie

4 vier

5 vijif

6 ses ayn

tway

dree

feor

feyf

zess
Datch.

7 zeven

8 acht

9 negen

10 tien

11 elf

12 twaalf
Pronunciation.

zayfe(r)n

$\mathrm{a}(\mathrm{h}) \operatorname{cht}$

nayge(r)n

teen

elf

twahlf 
Dutch. *Pronunciation.

13 dertien derr'teen

14 veertien fayrteen

15 vijftien feyfteen

16 zestien zesteen

17 zeventien zayfe(r)nteen

18 achttien a(h)chteen

19 negentien nayge(r)nteen

20 twintig twinte( $\mathrm{x})$ ch

21 een en twintig ayn $\mathrm{e}(\mathrm{r}) \mathrm{n}$

22 twee en - twinte $(\mathbf{r})$ ch

23 drie en

24 vier en

25 vijf en

26 zes en

27 zeven en -

28 acht en -

29 negen en -..

30 dertig derr'te(r)ch

40 veertig fayrte(r)ch

50 vijftig feyfte(r)ch

60 zestig zeste(r)ch
Dutch.

70 zeventig

80 tachtig

(

90 negentig nayge(r)nte(r)ch

100 honderd honder(r)t

$101-(e n)$ een

110 - (en) tien

120 - (en) twintig

130 - (en) dertig

200 tweehonderd

$1905\left\{\begin{array}{c}\text { duizend negen honderd doyze(r)nt nayge(r)n hon- } \\ \text { en vijf, or } \\ \text { negentienhonderd on vijf nayge(r)nteenhondert on }\end{array}\right.$

33. Ordinal Numbers. (Rangtelwoorden.)

400 vier
500 vijf
600 zes
700 zeven
800 acht
900 negen
1,000 duizen
2,000 twee
3,000 drie
10,000 tien
$1,000,000$ m
$d$ doyze(r)n
dert en
jf nayge(r)nt
(Rangteh

\begin{tabular}{|c|c|c|c|}
\hline The 1st & de eerste & $\mathrm{de}($ & ayrste $(\mathrm{r})$ \\
\hline$-\quad 2 n d$ & , tweede &, & twayde(r) \\
\hline$-3 r d$ & , derde & , & derrde $(r)$ \\
\hline$-\quad 4 \mathrm{th}$ & , vierde & , & feerde $(r)$ \\
\hline-5 th & "vijfde & " & feyfde(r) \\
\hline-6 th & , zesde & 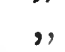 & zesde $(\mathrm{r})$ \\
\hline 7 th & ", zevende & ," & zayfe(r)nde(r) \\
\hline 8th & ,, achtste & ," & $\mathrm{a}(\mathrm{h}) \operatorname{chtste}(\mathrm{r})$ \\
\hline-9 th & , negende & , & nayge $(\mathrm{r})$ nde $(\mathrm{r})$ \\
\hline-10 th & , tiende & " & teende $(\mathrm{r})$ \\
\hline 11th & ,, elfde & ", & elfde $(r)$ \\
\hline$-12 \mathrm{th}$ & , twaalfde & , & twahlfde(r) \\
\hline-13 th & , dertiende & , & derrteende(r) \\
\hline 1 & , veertiende & , & fayrteende(r) \\
\hline
\end{tabular}

- Where pronunciation is omitted, it will be found given previously. 
English

the 15th

- 16th

- 17th

-18 th

- 19th

- 20th

-21 st

- 30th

- 40th

-50 th

- 60th

- 70th

-80 th

- 90th

- 100th

$-1,000$ th
Datch.

de vijftiende

", zestiende

,. zeventiende

, achttiende

", negentiende

", twintigste

", eenentwingtigste

,, dertigste

", veertigste

, vijftigste

,, zestigste

,, zeventigste

, tachtigste

,, negentigste

", honderdste

,, duizendste
Pronunciation.

de(r) feyf'teende(r)

, zess'teende(r)

", zayfe(r)nteende(r)

$\mathrm{a}(\mathrm{h}) c h^{\prime}$ teende(r)

nay'ge(r)nteende(r)

twin'te(r)gste(r)

ayn'e(r)ntwinte(r) $g$ -

derr'te(r)gste(r) [ste(r)

fayr'te(r)gste(r)

feyf'te(r)gste(r)

zess'te(r)gste(r)

zayfe(r)nte(r)gste(r)

ta $(\mathrm{h}) c h^{\prime} \operatorname{te}(\mathrm{r}) g \operatorname{ste}(\mathrm{r})$

nay'ge(r)nte(r)gste(r)

hon'dertste(r)

doy'ze(r)ndste(r)

\section{Collective and Fractional Numbers, \&c.}

(Verzamelnamen en Breuken.)

(For Grammatical Notes seo p. 81.)

Century

-, half-a-

couple (pair)

crowd

decado

double

dozen

a fifth

four-fifths

a gross

half

quarter(fourth part)

score

single, singly

tens

tenths

a third

three-fold

three-quarters eeuw $\mathrm{f}$.

een halve eeuw

paar $\mathrm{n}$.

gedrang n., menigte

tiental $\mathrm{n}$.

dubbel

dozijn $\mathrm{n}$.

een vijfde

vier vijfden

twaalf dozijn, gros n. twahlf dohzeyn',

helft $\mathrm{f}$.

vierendeel, kwart

een twintigtal

alleen

tienen, tientallen

tienden

een derde

drieroud

drie vierden helft [gross

$\mathrm{ay}^{\prime} \mathrm{w}$

ayn ha(h)lfe(r)

f. pahr $[\mathrm{ne}(\mathbf{r}) g \mathrm{te}(\mathbf{r})$

teenta $(\mathrm{h}) \mathrm{l}$

dub'be(r)l

dohzeyn'

ayn feyfde(r)

feer feyfde(r)

- fee're(r)ndayl,

kwa(h)rt

ayn twin'te(r) $g$ -

a(h)llayn' $\quad[\mathrm{ta}(\mathrm{h}) 1$ teenen, teenta(h) $1^{\prime}$ teende(r)n $\quad[\mathrm{le}(\mathrm{r}) \mathrm{n}$

ayn derrde(r)

dree'fowt

dree feerde(r)n 


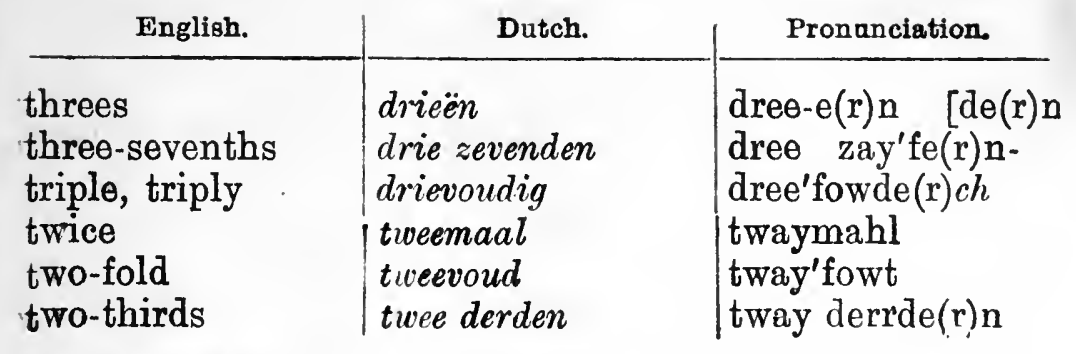

36. Adjectives.* (Bijvoegelijke Naamwoorden.)

(For Grammatical Notes see pages 71 to 76. )

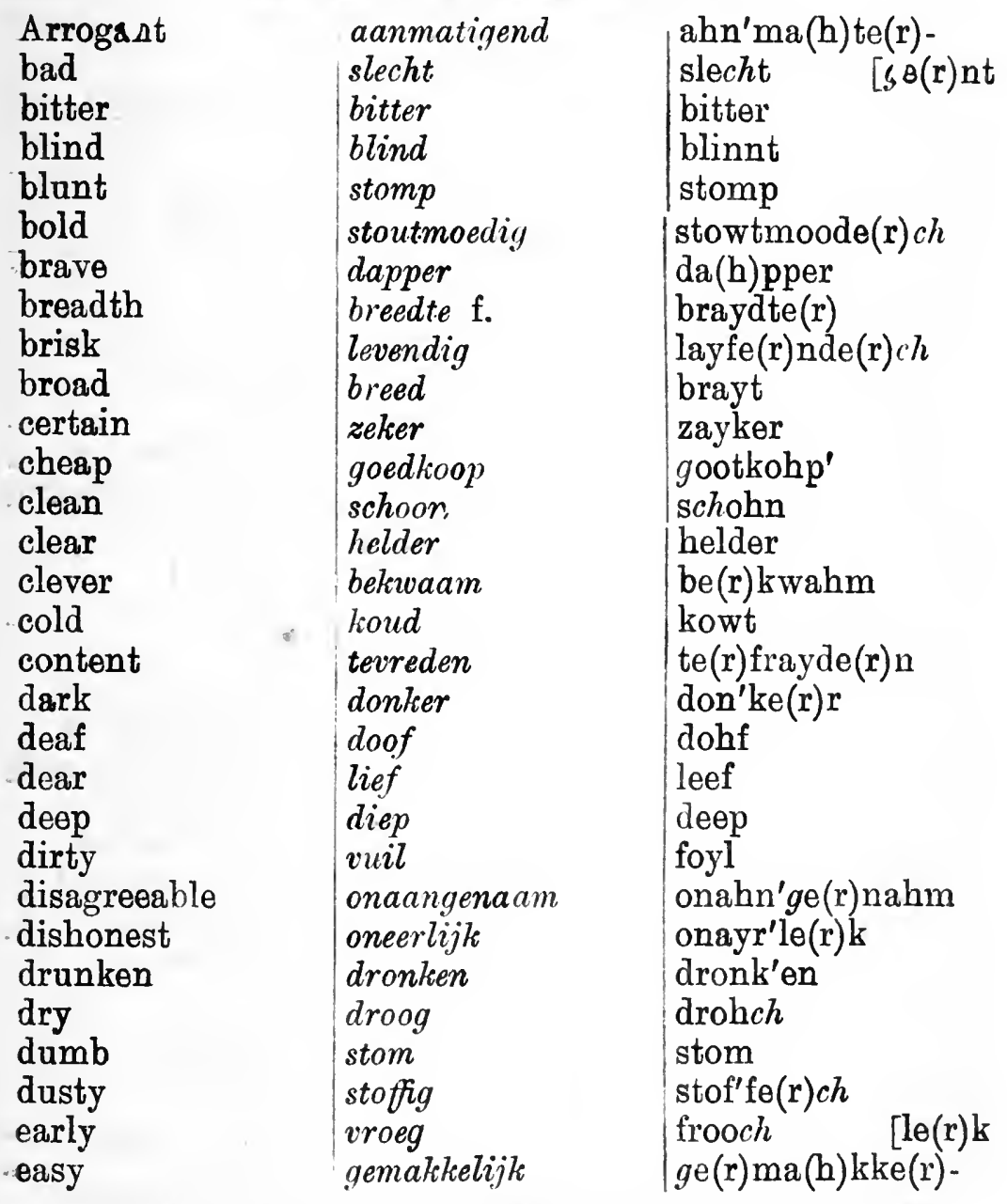

* Some Nouns of quality are here given for convenient reference. 
58

\begin{tabular}{|c|c|c|}
\hline Eng. & Dutch. & Pronunciation. \\
\hline $\begin{array}{l}\text { faithless } \\
\text { false } \\
\text { fat } \\
\text { fierce } \\
\text { fit } \\
\text { flat } \\
\text { foolish } \\
\text { free } \\
\text { frequent } \\
\text { fresh } \\
\text { full } \\
\text { gay } \\
\text { gentle } \\
\text { good } \\
\text { great } \\
\text { hairy } \\
\text { handsome } \\
\text { happy } \\
\text { hard } \\
\text { heavy } \\
\text { height } \\
\text { high } \\
\text { hollow } \\
\text { honest } \\
\text { hungry } \\
\text { ill } \\
\text { impolite } \\
\text { innocent } \\
\text { just } \\
\text { lame } \\
\text { large } \\
\text { late } \\
\text { lean } \\
\text { length } \\
\text { light } \\
\text { long } \\
\text { muddy }\end{array}$ & 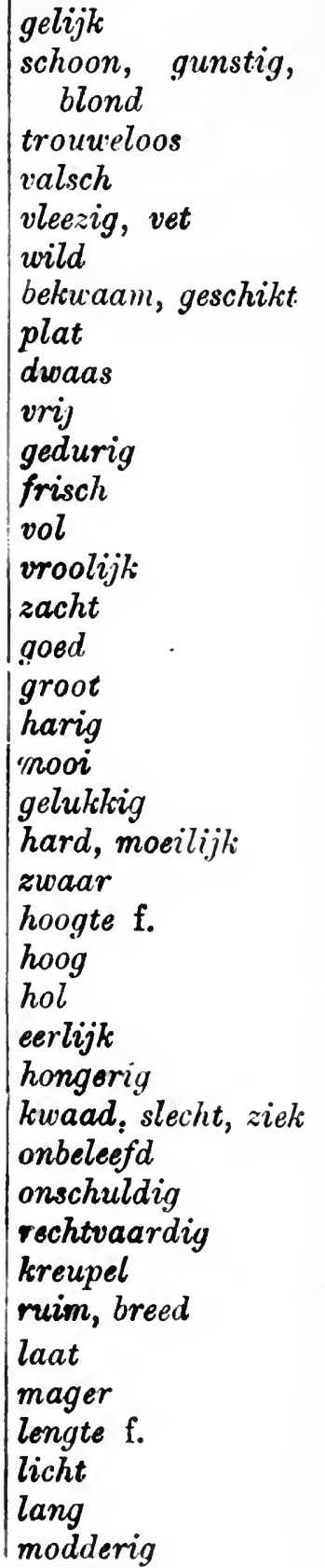 & $\begin{array}{l}\text { ge(r)leyk } \\
\text { schohn,gun'ste(r)ch, } \\
\quad \text { blont } \\
\text { trow'e(r)lohss } \\
\text { fa(h)lss } \\
\text { flay'ze(r)ch, fet } \\
\text { wilt } \\
\text { be(r)kwahm, ge(r). } \\
\text { pla(h)t [schikt. } \\
\text { dwahss } \\
\text { frey } \\
\text { ge(r)deure(r)ch } \\
\text { friss } \\
\text { fol } \\
\text { frohle(r)k } \\
\text { za(h)cht } \\
\text { goo't } \\
\text { groht } \\
\text { hahre(r)ch } \\
\text { moh'i } \\
\text { ge(r)luk'ke(r)ch } \\
\text { ha(h)rt, mooile(r)k. } \\
\text { zwahr } \\
\text { hohgte(r) } \\
\text { hohch } \\
\text { hol } \\
\text { ayrle(r)k } \\
\text { hong-e(r)re(r)ch } \\
\text { kwaht, glecht, zeek. } \\
\text { onbe(r)layft' } \\
\text { onschul'de(r)ch } \\
\text { rechtfahr'de(r)ch } \\
\text { kru(r)'pe(r)l } \\
\text { roym, brayt } \\
\text { laht } \\
\text { mahge(r)r } \\
\text { lengte(r) } \\
\text { licht } \\
\text { la(h)ng } \\
\text { modde(r)re(r)ch }\end{array}$ \\
\hline
\end{tabular}




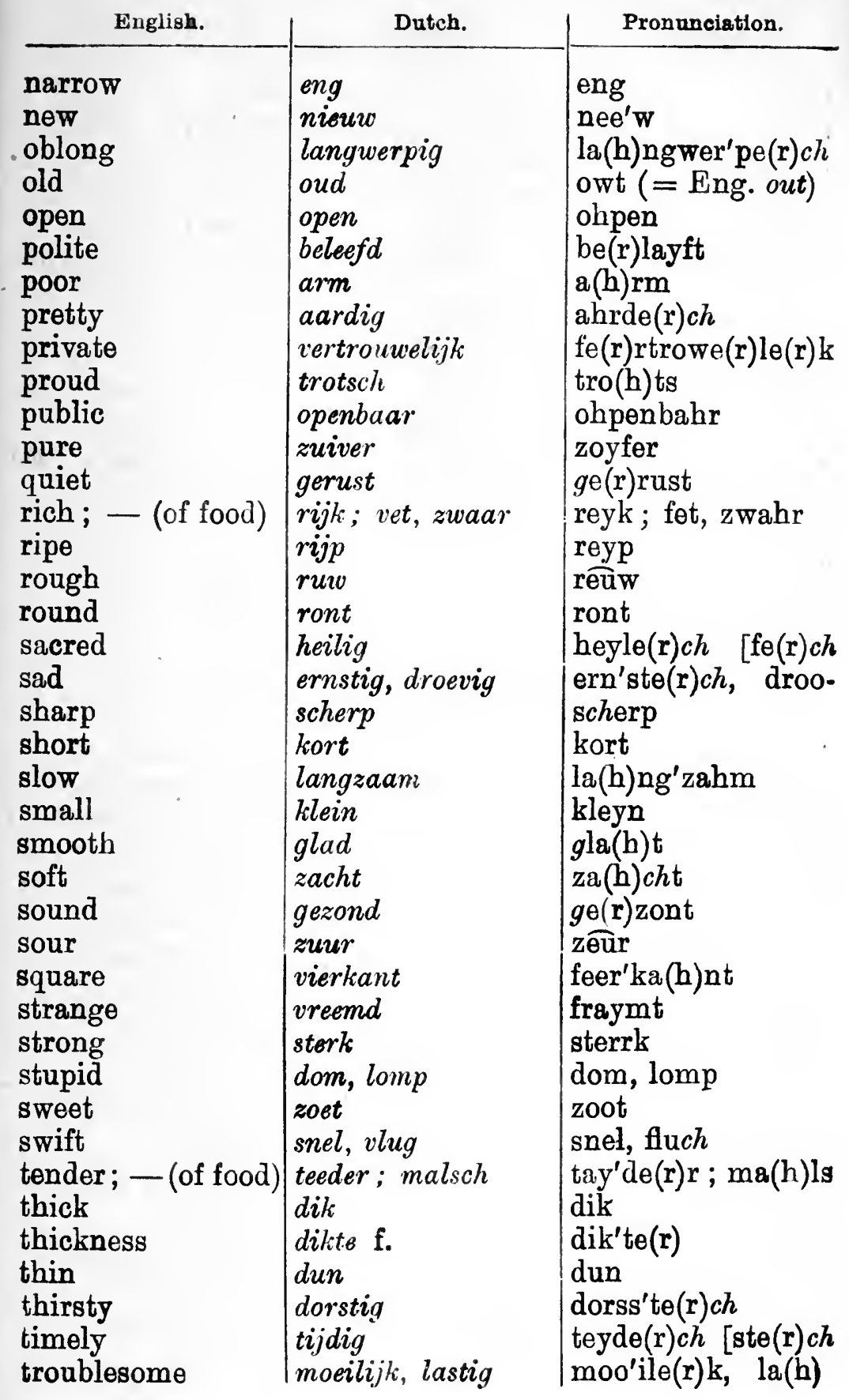




\begin{tabular}{|c|c|c|}
\hline English. & Dutch. & Pronunciation. \\
\hline true & waar & wahr \\
\hline ugly & leelijk & lay'le(r)k \\
\hline uncertain & onzeker & on'zayker \\
\hline unequal & ongelijk & on'ge(r)le(r)k \\
\hline unfit & ongeschikt & on'ge(r)schikt \\
\hline unsettled & onbestendig & onbe(r)sten'de(r)ch \\
\hline useful & nuttig & nut'te (r)ch \\
\hline vain & vergeefsch & fergayfss \\
\hline warm & warm & wa(h)rm \\
\hline weak & slap, zwak & sla(h)p, zwa(h)k \\
\hline well & wel, gezond & wel, ge(r)zont \\
\hline wet & nat, vochtig & $\mathrm{na}(\mathrm{h}) \mathrm{t}$, foch'te(r)ch \\
\hline $\begin{array}{l}\text { whole } \\
\text { wide }\end{array}$ & $\begin{array}{l}\text { geheel } \\
\text { wijd }\end{array}$ & $\begin{array}{l}\text { ge(r)hayl } \\
\text { weyt }\end{array}$ \\
\hline wild & wild & wilt \\
\hline wise & $w i j s$ & weyss \\
\hline young & jong & yong \\
\hline
\end{tabular}

36. Yerbs. (Werkwoorden.)

(For Grammatical Notes see pages 52 to 90. )

\begin{tabular}{|c|c|c|c|}
\hline $\begin{array}{l}\text { To accept } \\
\text { to admire } \\
\text { to affirm } \\
\text { to answer } \\
\text { to approve } \\
\text { to arrive } \\
\text { to ascend } \\
\text { to ask } \\
\text { to assure } \\
\text { to awake } \\
\text { to beat } \\
\text { to begin } \\
\text { to believe } \\
\text { to blame } \\
\text { to boil } \\
\text { to borrow } \\
\text { to breakfast } \\
\text { to bring } \\
\text { to build }\end{array}$ & & $\begin{array}{l}\text { aannemen } \\
\text { bewonderen } \\
\text { bevestigen } \\
\text { antwoorden } \\
\text { goedkeuren } \\
\text { aankomen } \\
\text { opklimmen } \\
\text { vragen } \\
\text { verzelieren } \\
\text { uekken } \\
\text { slaan, kloppen } \\
\text { beginnen } \\
\text { gelooven, vertrouwen } \\
\text { laken } \\
\text { koken } \\
\text { borgen, leenen } \\
\text { ontbijten } \\
\text { brengen } \\
\text { houwen }\end{array}$ & $\begin{array}{l}\text { ahn'nayme(r) } \\
\text { be(r)wonn'dere(r) } \\
\text { be(r)fes'te(r)ge(r) } \\
\text { a(b)nt'wohrde(r) } \\
\text { goo'tku(r)re(r) } \\
\text { ahn'kohme(r) } \\
\text { op }^{\prime} \text { klimme(r) }^{\prime} \\
\text { frah'ge(r) } \\
\text { fe(r)rzaykere(r) } \\
\text { weh'ke(r) } \\
\text { slahn, klop'pe(r) } \\
\text { be(r(gin'ne(r) } \\
\text { ge(r)lohfe(r), fer- } \\
\text { lahke(r) [trowe(r) } \\
\text { kohke(r) } \\
\text { borge(r), layne(r) } \\
\text { ontbey'te(r) } \\
\text { breng'e(r) } \\
\text { bow'e(r) }\end{array}$ \\
\hline
\end{tabular}


61

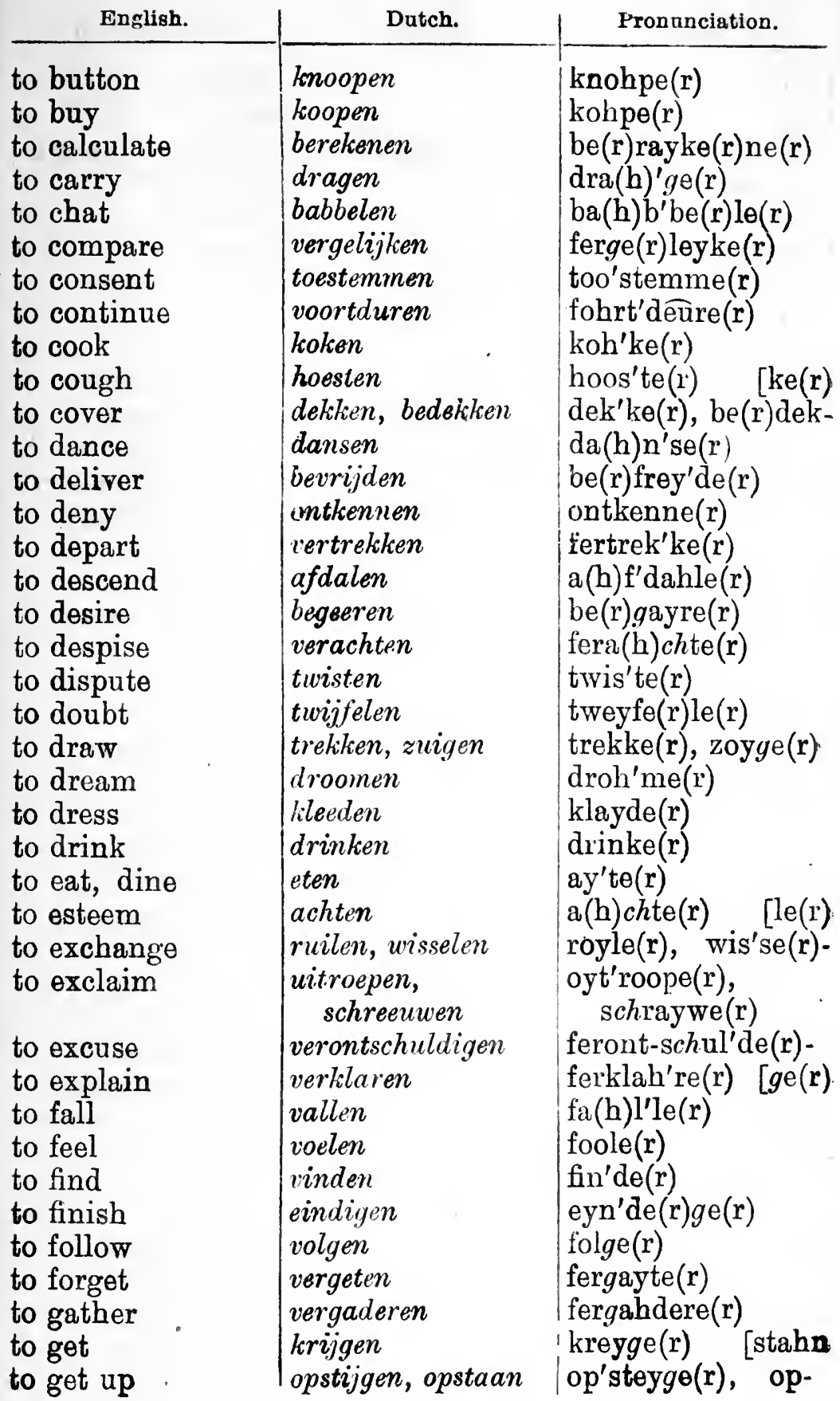


62

\begin{tabular}{|c|c|c|}
\hline & Datch. & Pronunciation. \\
\hline $\begin{array}{l}\text { to give } \\
\text { to go } \\
\text { to go in, - out } \\
\text { to grow } \\
\text { to hate } \\
\text { to hear } \\
\text { to bide } \\
\text { to honour } \\
\text { to hope } \\
\text { to ignore } \\
\text { to imagine } \\
\text { to imitate } \\
\text { to insult } \\
\text { to insure } \\
\text { to introduce } \\
\text { to jump } \\
\text { to know } \\
\text { to laugh } \\
\text { to learn } \\
\text { to lend } \\
\text { to lie down } \\
\text { to live } \\
\text { to dwell } \\
\text { to look for } \\
\text { to lose } \\
\text { to love } \\
\text { to marry } \\
\text { to meet } \\
\text { to mention } \\
\text { to be mistaken } \\
\text { to object } \\
\text { to offend } \\
\text { to offer } \\
\text { to open } \\
\text { to order } \\
\text { to pack up } \\
\text { to paint } \\
\text { to pardon } \\
\text { to pay } \\
\text { to plant } \\
\text { to pol }\end{array}$ & 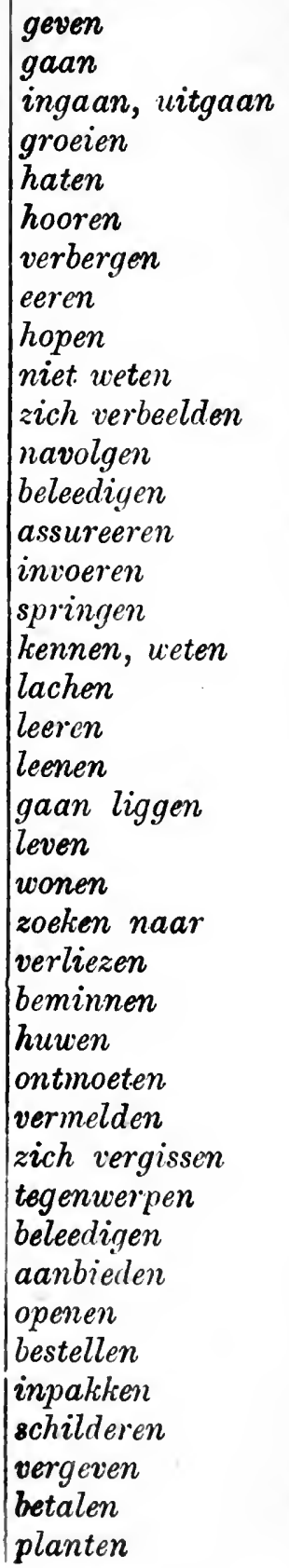 & 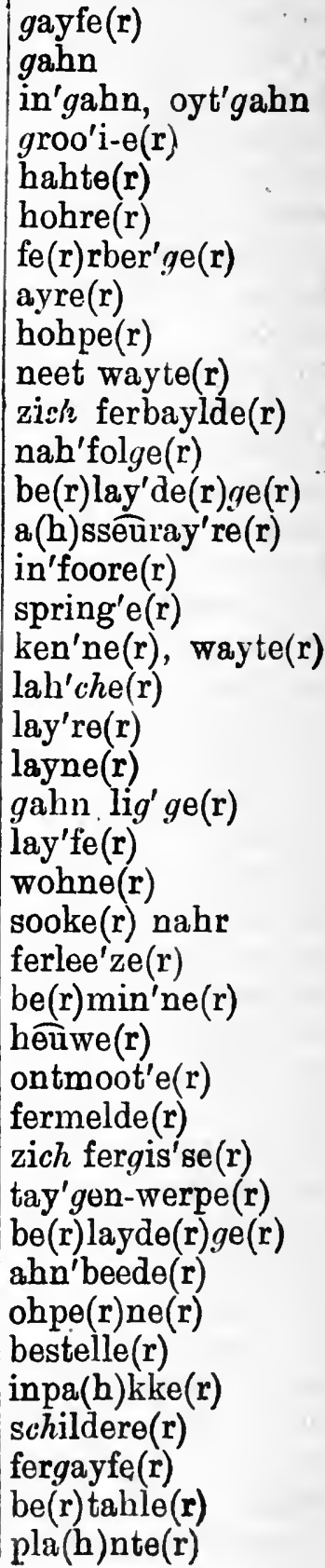 \\
\hline
\end{tabular}


63

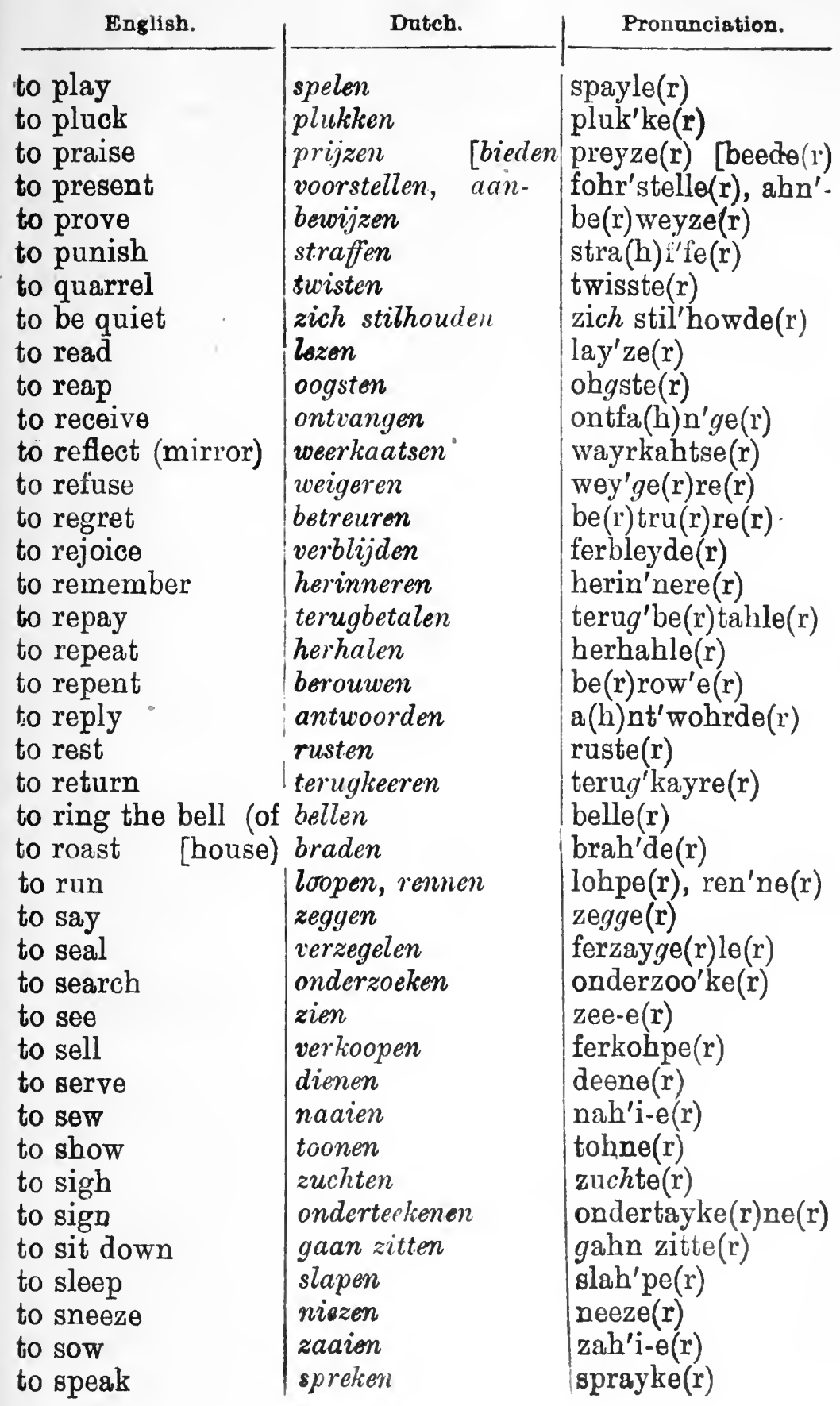




\begin{tabular}{|c|c|c|}
\hline English. & Datch. & Pronunciation. \\
\hline to & spinnen & spinne(r) \\
\hline & bederven & be(r)derfe(r) \\
\hline al & stelen & stay'le(r) \\
\hline$d y$ & studeeren & stenday're(r) \\
\hline$\theta$ & nemen & nayme $(r) \quad[k e(r)$ \\
\hline te & proeven, smaken & 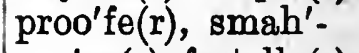 \\
\hline & zeggan, vertellen & $\mathrm{ze} g^{\prime} g \mathrm{e}(\mathrm{r})$, fertelle \\
\hline ank & danken & $\mathrm{da}(\mathrm{h}) \mathrm{n}^{\prime} \mathrm{ke}(\mathrm{r})$ \\
\hline$k$ & denken & den'ke(r) \\
\hline to be thirsty & dorst hebben & dorst hebbe(r) \\
\hline row & werpen & wer'pe(r) \\
\hline to & raken & rahke(r) \\
\hline slate & vertalen & fe(r)rtah'le(r) \\
\hline to & reizen & reyze $(\mathrm{r})$ \\
\hline ton & Iosknoopen & loss'k-nohpe(r) \\
\hline over & ontblooten & ontblohte(r) \\
\hline erstand & verstaan & fe(r)rstahn \\
\hline ack & (ont-), uitpakken & (cnt-), oyt'pa \\
\hline to & uasschen & wa(h)s'se(r) [ke(r). \\
\hline to $\nabla$ & weenen, schreien & wayne $(\mathrm{r}), \ldots \mathrm{scl}$ \\
\hline igh & wegen & way'ge(r) \\
\hline & willen & wil'le(r) \\
\hline to wish & wenschen & wens'se $(r) \quad[\operatorname{de}(r)$ \\
\hline to & werken, arbeiden & wer'ke(r), a(h)r'bey- \\
\hline to $\mathrm{w}$ & vereeren, aanbidden & ferayre $(r)$, \\
\hline $\begin{array}{l}\text { to wrap } \\
\text { to write }\end{array}$ & $\begin{array}{l}\text { wikkelen } \\
\text { schrijien }\end{array}$ & $\begin{array}{l}\text { wik' } \mathrm{ke}(\mathrm{r}) \mathrm{le}(\mathrm{r}) \\
\text { schreyfen }\end{array}$ \\
\hline
\end{tabular}

\section{Adverbs, Conjunctions, and Prepositions.}

(Bijwoorden, Voegwoorden, Voorzetsels.)

(For Grammatioal Notes see pages 91 and 02.)

\begin{tabular}{|c|c|}
\hline $\begin{array}{l}\text { About } \\
\text { above } \\
\text { above all } \\
\text { accordingly } \\
\text { after } \\
\text { afterwards } \\
\text { again } \\
\text { against }\end{array}$ & $\begin{array}{l}\text { om, omtrent } \\
\text { boven } \\
\text { boven alles } \\
\text { overeenkomstig, } \\
\quad \text { volgens } \\
\text { achter, na } \\
\text { naderhand, daarna } \\
\text { weder } \\
\text { tegen }\end{array}$ \\
\hline
\end{tabular}
om, omtrent' boh'fen boh'fen a(h)l'less ohferaynkom' - ste(r)ch, folgens a(h)ch'ter, nah nahderha(h)nt', way'der dahrnah tay'gen




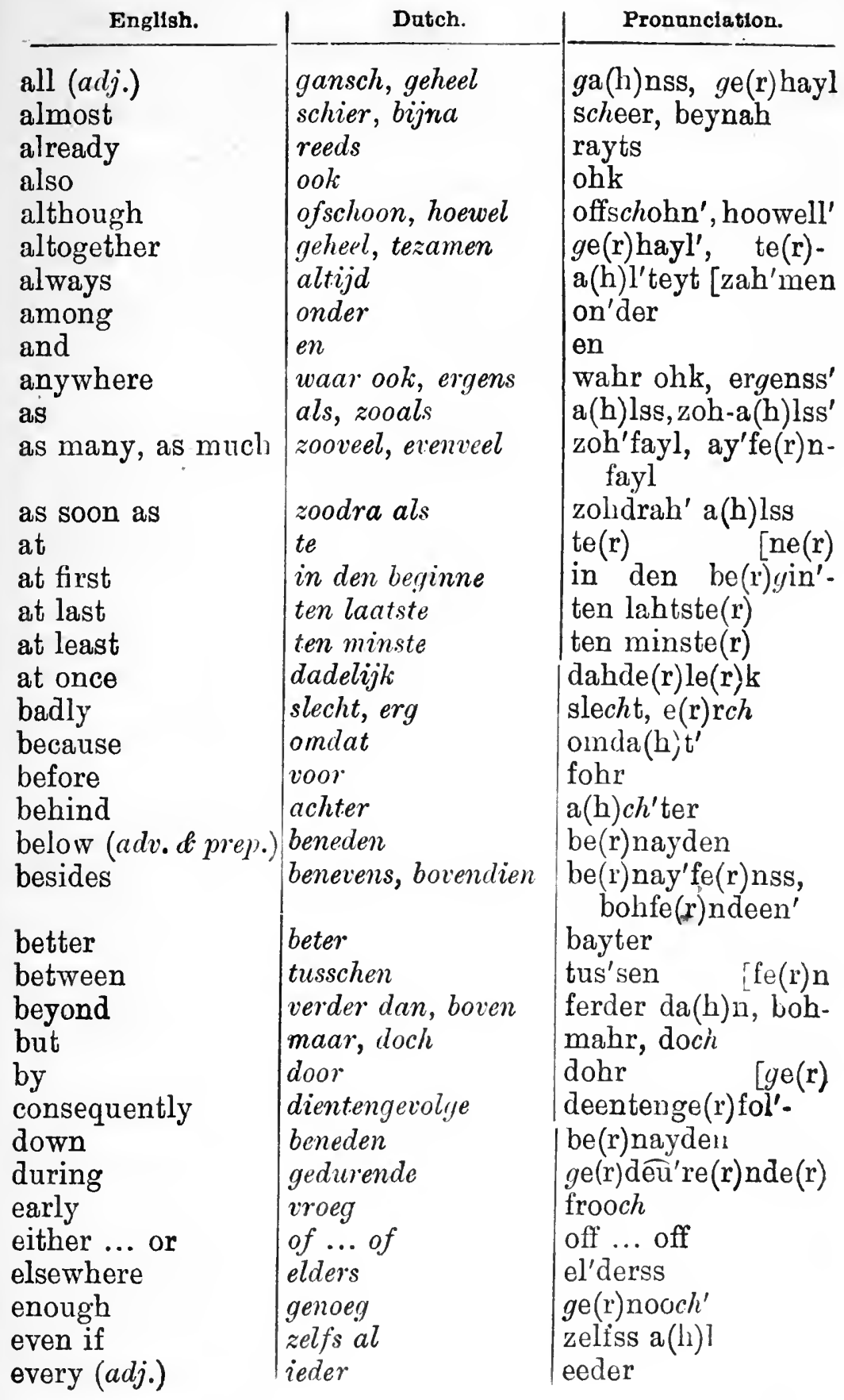




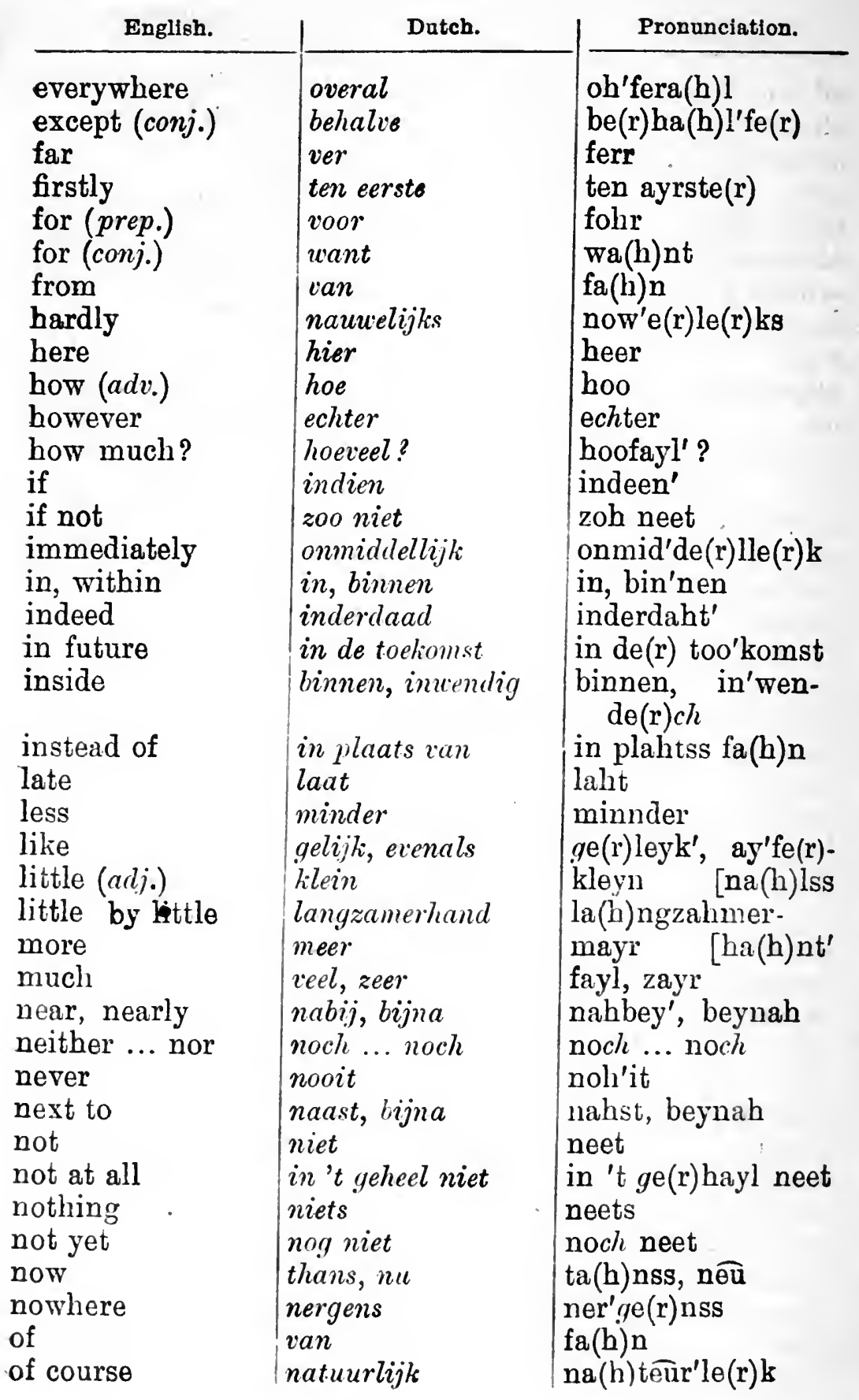




\begin{tabular}{|c|c|c|}
\hline & & \\
\hline $\begin{array}{l}\text { often } \\
\text { on } \\
\text { on the contrary } \\
\text { on the left } \\
\text { on the opposite side } \\
\text { on the right } \\
\text { on this side } \\
\text { once } \\
\text { only (adj.) } \\
\text { - (adv.) } \\
\text { opposite } \\
\text { or } \\
\text { otherwise (adv.) } \\
\text { outside } \\
\text { over } \\
\text { perhaps } \\
\text { presently, soon } \\
\text { previously } \\
\text { quickly } \\
\text { rather } \\
\text { same (the) (arlj.) } \\
\text { scarcely } \\
\text { secondly } \\
\text { seldom } \\
\text { since (prep.) } \\
\text { - (conj.) } \\
\text { so } \\
\text { some (adj.) } \\
\text { sometimes } \\
\text { soon } \\
\text { suddenly } \\
\text { then (adv. } \& \text { conj.) } \\
\text { thence } \\
\text { thenceforth } \\
\text { there } \\
\text { therefore } \\
\text { thirdly } \\
\text { through } \\
\text { ond }\end{array}$ & $\begin{array}{l}\text { dikwijls } \\
\text { op, aan } \\
\text { integendeel } \\
\text { links } \\
\text { aan de overzijde } \\
\text { rechts } \\
\text { aan deze zijde } \\
\text { eens } \\
\text { eenng } \\
\text { alleen, slechts } \\
\text { tegengesteld } \\
\text { of } \\
\text { anders } \\
\text { buitenzijde f. } \\
\text { boven } \\
\text { misschien } \\
\text { straks, dadelijk } \\
\text { vooraf } \\
\text { schielijk } \\
\text { liever; eerder } \\
\text { zelfde } \\
\text { nauwelijks } \\
\text { ten tweede } \\
\text { zelden } \\
\text { sedert, sints } \\
\text { sedert } \\
\text { zoo, dus } \\
\text { eenige } \\
\text { somtijds } \\
\text { weldra, vroeg, } \\
\text { spoedig } \\
\text { plotseling } \\
\text { dan } \\
\text { van daar } \\
\text { van dien tijd af } \\
\text { daar } \\
\text { daarom } \\
\text { ten derde } \\
\text { door } \\
\text { aldus } \\
\text { ald } \\
\text { and }\end{array}$ & $\begin{array}{l}\text { dik'weylss } \\
\text { op, ahn } \\
\text { intay'gendayl } \\
\text { links } \\
\text { ahn de(r) oh'ferzey- } \\
\text { rechtss } \\
\text { ahn dayze(r) zey- } \\
\text { aynss [de(r) } \\
\text { ay'ne(r)ch } \\
\text { a(h)llayn', slechts } \\
\text { tay'ge(r)ngesstelt } \\
\text { off' } \\
\text { a(b)n'derss } \\
\text { boy'te(r)nzeyde(r) } \\
\text { boh'fen } \\
\text { misscheen' } \quad \text { le(r)k } \\
\text { stra(h)ks, dahde(r)- } \\
\text { fohra(h)f' } \\
\text { scheele(r)k } \\
\text { leefer, ayrder } \\
\text { zelfde(r) } \\
\text { now'e(r)le(r)kss } \\
\text { ten twayde(r) } \\
\text { zelden } \\
\text { say'derrt, sintss } \\
\text { say'derrt } \\
\text { zoh, duss } \\
\text { ayne(r)ge(r) } \\
\text { somteyts' } \\
\text { weldrah, frooch, } \\
\text { spoode(r)ch } \\
\text { plotsse(r)ling } \\
\text { da(h)n } \\
\text { fa(h)n dahr } \\
\text { fa(h)n deen teyt } \\
\text { dahr } \\
\text { dahr'om } \\
\text { ten derrde(r) } \\
\text { dohr } \\
\text { a(h)lduss' }\end{array}$ \\
\hline
\end{tabular}




\begin{tabular}{|c|c|c|}
\hline English. & Dutich. & Pronunciation. \\
\hline to & aan, naar & ahn, nahr \\
\hline too & ool & ohk \\
\hline too much & te veel & te(r) fayl \\
\hline together & tegelijk & te(r)ge(r)leyk \\
\hline towards & jegens & yay'ge(r)nss \\
\hline under & onder & on'der \\
\hline undoubtedly & ongetuiiffeld & on'getweyfelt \\
\hline unless & tenzij & ten-zey' \\
\hline until & tot & tot \\
\hline up & op & op \\
\hline upon & $o p$, aan & op, abn \\
\hline very & $z e e r$ & zayr \\
\hline well & goed, zeer & goot, zayr \\
\hline when & wanneer, toen & wa(h)nnayr', toon \\
\hline where? & waar? & wahr? \\
\hline while & terwijl & te(r)rweyl \\
\hline why? & waarom? & wahr'om? \\
\hline willingly & gaarne & gahr'ne(r) \\
\hline with & met. & met \\
\hline with, near & na, met & nah, met. \\
\hline within ( $a d v$. ) & binnen & bin'nen \\
\hline without (prep.) & zonder & zon'der \\
\hline yet & $n o g$, toch & noch, toch \\
\hline
\end{tabular}

\title{
MARLBOROUGH'S \\ Travellers' Practical Manuals of Conversation.
}

\author{
SUBJECTS ARRANGED ALPHABETICALLY.
}

Containing Travel Notes, Classified Conversations-Railway, Steamer, Hotel, Luggage, Customs, Motoring, Cycling, \&c., Money, Weights and Measures. Dictionary in four languages of 'Words in Daily Use.

No. 1.-ENGLISH, FRENCH, GERMAN and ITALIAN. (In one book.) No. 2.-ENGLISH, FRENCH, GERMAN and DUTCH. (In one book.) Crown 12mo, $152 \mathrm{pp}$. Wrapper, $1 / 9$ net each : Cloth. 3/-net each.

\section{German Self = Taught, Grammar with Key.}

BY W. E. WEBER, M.A.

For learning the language by the Natural Method, with Phonetic Pronunciation. Containing Notes on Articles, Gender, Accents, \&c. ; Classifled Vocabularies; Conversational Phrases and Sentences ; Naval, Military and Commercial Terms; Tables of Money, \&c. A course of simple and practical lessons in the Rules of Grammar exemplified with Model Sentences, Exercises and Reading Lessons in German Characters. Facsimile German Hand-writing, Alphabetical Vocabulary. \&c.

crown $8 \mathrm{cos} 332 \mathrm{pp}$. Green Cloth, 6:- net.

London: E. MARLBOROUGH \& Co., Publishers, 51 0ld Bailey, E.C.4. 


\section{ELEMENTARY GRAMMAR.}

The Dutch language has ten parts of speech:-

\section{The Articles. (Lidwoorden.)}

There are two Articles, the Definite and the INDEwivite.

There are three Genders-masculine (M.), feminine (F.), and neuter (N.), and four Cases-Nominative (N.), Genitive (G.), Dative (D.), and Accusative (A.)

General RULE. - Each gender has its own form of declension in the singular; in the plural there is no distinction between the genders.

\section{DECLENSION.}

\section{The Definite Article.}

$\begin{array}{lllll}\text { Singular: } & \text { M. } & \text { F. } & \text { N. } & \text { Plural for all genders. } \\ \text { N. the } & \text { de } & \text { de } & \text { het } & \text { de } \\ \text { G. of the } & \text { des } & \text { der } & \text { des } & \text { der } \\ \text { D. to the } & \text { den } & \text { de } & \text { het } & \text { den } \\ \text { A. the } & \text { den } & \text { de } & \text { het } & \text { de. }\end{array}$

\section{The Indefinite Article.}

N. a, angular: M.

G. of a, of an eens

D. to a, to an $\operatorname{een}\left(e n^{*}\right)$

A. a, an

$\operatorname{een}\left(e n^{*}\right)$

$\begin{array}{ll}\text { F. } & \text { N. } \\ \text { ecn }(e) & \text { een } \\ \text { eener } & \text { eens } \\ \text { een }\left(e^{*}\right) & \text { een } \\ \text { een }\left(e^{*}\right) & \text { een. }\end{array}$

* These endings are never used in the spoken language, and seldom in the written.

NoTE.-The definite article is often used in Dutch, where there is no article

in English, as: Van de hand in de tand, from hand to mouth.

There are phrases, however, in which the definite article used in

English is not used in Dutch, as : Piano spelen, to play the piano.

\section{The Noun. (Het zelfstandig naamwoord.)}

\section{GENDER.}

\section{Masculine Nouns are:-}

(a) Names of males, when there is a separate name for the male; when not, names of great animals are masculine, as olifant (m.), elephant, and those of small animals are feminine, as muis (f.), mouse.

(b) names of seasons [EXCEPT Lente (f.), Spring], months, and days [EXCEPT the compounds with month and year: Meimaand (f.), month of May; voorjaar (n.), spring.

(c) names of trees [EXCEPT Linde (f.), lime tree, and tamarinde (f.), tamarind], stones, coins [EXCEPT munt (f.), coin; guinje (f.), guinea; pistool (f.), pistole; pond (n.), pound], and mountains. 
(d) nouns of one syllable indicating motion or action, as houro, stab; greep, grasp, \&c. Such words are feminine when they indicate objects having any relation to the action; for in. stance, $\operatorname{trap}(\mathrm{m}$.$) , kick ; trap (f.), staircase.$

(e) nouns ending in -aar, -aard, -enaar, -erd, -enier, -el, -em, -sem, $-l m,-r m,-e r$, and most words ending in -en, as

lessenaar, desk

luiaard, sluggard geweldenaar, tyrant mosterd, mustard kruidenier, grocer

beitel, chisel

adem, breath

bloesem, blossom halm, stalk storm, storm looper, stair-carpet regen, rain.

Exceptions: Griffel (f.), slate pencil; schoffel (f.), hoe ; scherm (n.), screen; palm (f.), palm of the hand; uniform (f.), uniform; helm (f.), broom.

$(f)$ all words ending in -ing and -ling (when not derived from verbs), as ketting, chain; krakeling, cracknel.

(g) nouns ending in -dom, when denoting a state, as wasdom, growth.

For Exceptions see under Neuter $(g)$.

Feminine are :-

(a) Names of females (when there is a separate name for the female), flowers, vegetables, fruits, grains, foreign products, materials (when not neuter), ships (EXCEPT those ending in -er), letters of the alphabet, ciphers, figures, musical notes, interyals, and foreign instruments [EXCEPT doedelzak (m.), hoorn (m.), triangel (m.)]

Note.-1. Names of flowers formed from names of other things take the gender of the latter, as goudenregen (m.), aronskelk (m.)

2. Names of fruits ending in oen, ling, el, and er are mascnline; e.g., citroen (m.), pippeling (m.), eikel.(m.), aker (m.)

3. Foreign names of fruits ending in $e l$ and $e r$ are feminine; e.g., komkommer (f.), amandel (f.)

(b) nouns ending in $-e,-d e$, -te, denoting inanimate objects, as koude, cold; hoogte, height.

Exceptrons: Vreie (m.), peace; einde (n.), end; and the collective nouns ending in -te, which are Neuter.

(c) nouns formed with the suffixes -heid, -nis, -teit, as goedheid, goodness; beeltenis, image ; majesteit, majesty.

ExCEPT Vonnis (n.), sentence; getuigenis (n.), testi. mony; concilie (n.), council; evangelie (n.), gospel.

(d) all nouns ending in -ie, -ick, -age, -ij, -ei, and -uw, as melodie, melody slijtage, wear and tear lei, slate fabriek, manufactory schilderij, painting schaduw, shade.

(e) Yerbal nouns in -ing, -st, and -t, as belooning, reward; kunst, art; vaart, canal.

EXCEPT Dienst (m.), service; last (m.), load; schrift (n.), writing [Schrift(f.) is (Holy) Scripture]; wicht (n.), weight. 
(f) Words in -schap, when denoting a state or a collection, that are not neuter [see $(h)$ under Neuter], as blijdschap, joy; verwantschap, relation.

\section{Neuter are:-}

(a) The names of young animals, and those that indicate a whole class, as kalf, calf ; zwijn, swine [EXCEPT Hond (m.), dog].

(b) Collective nouns, as leger, army; volk, people.

Exceptions: Stad (f.), town ; trits (f.), threes.

(c) The names of materials from which objects are manufactured, as barnsteen, amber; goud, gold.

Exceptrons: Materials which are feminine, and the following masculine nouns:-
azijn, vinegar
honig, honey
inkt, ink
mosterd, mustard
room, cream
wijn, wine.

(d) The infinitive mood of verbs, and all parts of speech usect as nouns, as het schoone, the beautiful [EXCEPT adjectives. used as names of persons].

(e) The names of countries, towns, and villages.

EXCEPTIONS: Geographical names which are preceded by the article de are Feminine, as de dorre Sahara, the barren Sahara [ExcePr Den Haag, the Hague; e.g., het bloeiende Den Haag, the prosperous Hague].

(f) The names of diminutives, as het kindje, the little child.

(g) Words ending in -dom, when denoting a collection or a country, as vorstendom, principality.

Exceptions: See under Masculine $(g)$.

(h) Words ending in -schap, when expressing a dignity, occupation, or territory, as koningschap, royalty.

Exceptions: See under Feminine $(f)$.

i) All words ending in -sel, as blauwsel, blue [EXCEPT Stijfsel, starch].

(j) Nouns formed from the stem of a yerb with the inseparable prefixes be-, ge-, onder-, ont-, ver-.

Exceptions: Ontvang (m.), reception; verkoop (m.), sale; verbouw (m.), growth.

NoTs.-1. Compound nouns have the gender of the principal word, as hofkerk (f.), Court chapel [EXCEPI kerkhof (n.), gravejard, derived from hof (m.), garden; tijdslip (n.), epoch].

2. There are some nouns which have different genders, with different meanings, as helm (m.), helmet, helm (f.), plant;

blite (m.), look, blik (n.), dustpan; and many others. 


\section{DECLENSION OF NOUNS.}

There are two declensions, termed the Strong and the Weak.

Most nouns belong to the Strong, the Genitive ending in $s$. Those belonging to the Weak end in en in the Genitive.

General Roles.-1. Feminine nouns always remain unchanged throughout the singular, and their declension is indicated only by the article or pronoun.

Exceptions : Family and proper names in the Genitive preceding another noun take $s$ or 's, as moeders verjaardag, mother's birthday; Maria's boeken, Mary's books.

Should a pronoun or an adjective precede such expressions, the nominative is used, as mijn moeders verjaardag, my mother's birthday.

2. Masculine and Neuter nouns form their Genitive Singular in $[e] s$ (Strong declension), or in [e] $n$ (Weak declension).

The following Masculine nouns belong to the Weak declension:

mensch, man vorst, prince paus, pope heer, lord

graaf, count hertog, earl prins, prince profeet, prophet; also the Neuter noun hart, heart, and all Adjectives used as masculine and neuter Nouns and ending in $\mathbf{e}$, as het goede, the good. Notr.-The Genitive and Dative are very seldom used in speaking Dutch;

in their place the Accusative is used, with the prepositions van, of, and aan, to, as van den valder, of the father, instead of des vaders.

3. The Accusative always has the same form as the Nomina. tive, and the Dative generally has also.

\section{Formation of Plural.}

The plural of nouns is formed by adding $n$, en, or $s$ in the four cases, and is the same in all genders.

1. Nouns ending in el, em, en, er, aar, ier, aard, erd, age, and in a LONG rowel take $\mathbf{s}$, as also all diminutives. Words borrowed from foreign languages take $s$, as bureaus. The Dutch words ra, via, and eega, ending in a long vowel, are in the plural raas, theels : vlaas, custards ; eegaas, spouses.

2. Nouns ending in $e$ take $\mathbf{n}$. Words ending in ie take $\mathbf{s}$ or $\mathbf{n}$ when the accent is not on ie, as provincies or provincienn. They cake en when ie has the accent; e.g., melodieën. Words ending in ee take en, as zbe-zeeën.

3. All nouns ending in a hissing sound or in a diphthong take en, as huis (house), huizen, bakkerij (bakery), bakierijen; aLso those ending in and, dom, eel, in, ing, ik, rik, schap, $u w$.

Words ending in or take s or en, as professors or professoren. Words ending in ier and eur take $\mathbf{s}$ when they denote persons, and en when they denote things, as portiers, door-keepers; portieren, coach-doors.

Some words take either, as zoons or zonen, sons; smids or smeden, smiths; gemaals or gemalen, husbands.

4. When the final consonant of a noun is preceded by a short rowel, the consonant is doubled in the plural, as rat (f.), rat-pl. 
ratten; EXCEPT when the short rowel of the singular changes into a long vowel in the plural, as weg (m.), road-pl. wegen.

5. The final $s$ in many nouns is changed into $z$, and the final $f$ into $v$. This is always the case when these consonants are preceded by a long vowel, as haas (m.), hare-pl. hazen; wolf (m.), wolf-pl. wolven.

6. Nouns ending in man and denoting a profession, change this into lieden in the plural, as werkman (m.), workman-pl. werklieden; Oranjeman, Noorman, blindeman, as not denoting a profession, are in the plural Oranjemannen, Noormannen, blindemannen; and those ending in heid change into heden, as schoonheid (f.), beauty-pl. schoonheden.

7. Some nouns change their root vowel in the plural, as schip (n.), ship-pl. schepen; stad, town-pl. steden.

8. There are many nouns having no plural, as in English, whilst others are used only in the plural; thus,

(a) Names of materials and most abstract nouns have no plural in Dutch.

(b) Nouns denoting measure, weight, and number are used in the Singular, although accompanied by plural numerals, as tien yoet lang, мот tien voeten lang; but wichtjes, maatjes, rijksdaalers, stuivers, kwartjes, cents.

Hij heeft een intiomen van duizend gulden (collectively). He has an income of a thousand guilders.

Itk heb hier drie guldens (singly).

I have three guilders here.

NoTs.-1. Some Neuter nouns take er before the plural-ending, and a few take er-en as well as er-s, as ei (sing.), eieren (pl.); hoen (sing.), hoenderen or hoenders (pl.) The plural in eren is more sublime than that in ors. Ex., Hier zijn arie appels (Here are three apples). Mijn woorden waren als gouden appelen in zilveren schalen (Prov. $x \mathrm{x}$. 11).

2. The plural of vioo (f.), flea, is vlooien, and of koo (f.), cow, koeien.

\section{Examples of Declension.}

De vader, the father; de moeder, the mother; het lind, the child.

Singular.

\begin{abstract}
Mas.
N. de vader

G. des vaders

D. den vader

A. den vader
\end{abstract}

N. de vaders

G. der vaders

D. den vaders

A. de vaders
Fem. de moeder der moeder de mocder de moeder

\section{Plural.} de moeders der moeders den moeders de moeders
Nent.

het lind

des linds

het kind

het kind.

de kinderen

der kinderen

den kinderen

de kinderen. 
Strong Dechension.

Een mond, a mouth; een vrouw, a wife; een veld, a field. Singular.

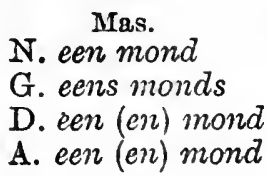

N. monden

G. monden

D. monden

A. monden
Fem.

eene vrouw

eene vrouw

eener (eene) vrouw

eene vrouw

Plural.

vrouwen

vrouwen

vrouwen

vrouven
Neut. een veld eens velds een veld een veld.

velden velden velden velden.

\title{
Weak Declension.
}

Singular.

N. de bode, the messenger

$\mathrm{G}$. des boden

D. den bode

A. den bode

\author{
Neut. \\ het hart, the heart \\ des harten \\ het hart \\ het hart.
}

Plural.

N. de boden, the messengers

G. der boden

D. den boden

A. de boden

de harten, the hearts

der harten

den harten

de harten.

\section{The Adjective. (Het bijvoeglijk Naamuoord.)}

For list of Adjectives, see pp. 57 to 60 ; Numerals, 54 to 57 .

The Adjective may be used as an attribute, as de goede man, the good man, or as a predicate, as de man is goed, the man is good.

When used as a predicate and placed after a noun it is not declined, but retains the same form in all genders and numbers.

As an attribute an adjective is declined as follows :-

De goede man, the good man;

Mas.

N. de goede man

G. des goeden mans

D. den goeden man

A. den goeden man

$\mathrm{N}$. de goede mannen

G. der goede mannen

D. den goeden mannen

A. de goede mannen de goede vrouw, the good woman;

Singular.

Fem.

de goede vrouw

der goede vrouw

de goede vrouw

de goede vrouw

Plural.

de goede vrouwen

der goede vrouwen

den goeden vrouwen

de goede vrouwen het goede kind. the good child.

Neut. het goede kind des goeden kinds het goede kind het goede kind.

de goede kinderen der goede kinderen den goeden kinderen de goede kinderen. 
Notw.-1. Instead of the masculine and feminine genitive, van is generally used, as van den blinde (m.), of a blind man; van de blinde ( $f$.), of a blind woman. In the neuter genitive van is always used, as van het goede.

2. If an article or a pronoun (not a personal pronoun) precedes, the adjective always has $(e) n$ when the articie or pronoun ends in $s$ or $n$.

3. Adjectives not preceded by any word, or preceded by a word with indefinite signification, are not declined in the nominative and accusative neuter, as ieder nieun huis, each new house.

4. The Snperiatives and the Ordinal Numbers take $e$. Adjectives preceding Masculine nouns drop the ending $\theta$ in the nominative, dative, and accusative, if they are used with een and if the quality refers to the profession, as een groote man, a tall man; een groot man, a great man.

\section{The following Adjectives are not declined:-}

(a) Those ending in er, and derived from geographical names.

(b) All adjectives of material ending in en; but those ending in sch, like lakensch, duffelsch, and neteldoeksch, are declined.

(c) Those ending in en.

(d) The adjectives rechter and linker.

(e) All adjectives used as predicates or placed after the noun; e.g., God is goed, God Almachtig. Also in phrases like $Z$ oo groot eene opoffering, so great a sacrifice.

(f) The comparatives, especially those of two, or more than two, syllables, as dapperder mannen, braver men; uitvoeriger berichten, more detailed pieces of information.

\section{Comparison of Adjectives.}

There are three degrees of comparison-the Positive, the Comparative, and the Superlative.

1. The Positive has no inflectional termination.

2. The Comparative is formed by adding or to the Positive; $r$ only is added when the adjective ends in $e$.

Note.-1. For the sake of euphony Positives ending in $r$ take $d$ before er, as zwaar, heavy; zwaarder, heavicr.

2. The Comparison of Equality is expressed by zoo...als, or even (evenzoo)...als, (just) as...as; c.g.,

Ik ben zoo groot als $h i j$, I am as tall as he.

$H i j$ is even (zOo) vergerioge als $w i j, \mathrm{He}$ is (just) as contented as we.

3. The Comparison of Superiority is expressed by meer...dan, more ..than, or eerder...dan, rather than, or by the comparative only, followed by dan; e.g.,

Hij is meer handig dan geleerd, He is more clever than learned.

Deze leerling is eerder vlug dan vlijtig,

Jan is grooter dan Piet,

This pupili is quick rather than industrious.

John is taller than Peter.

8. The Superlative is formed by adding st to the Positive, as groot, great; grootst, greatest.

Notr. - 1. If the Positive ends in $e$ this letter is dropped, as blijde, gay blijist, gayest.

2. If the Positive ends in $s$ or sch $t$ oniy is added, as wijs, wise-wijst, wisest; frisch, fresh-frischt, freshest. 
3. The Superlatize of Eminence is usually expressed by an adverb preceding the Positive, as uiterst klein, extremely small; hoogst gelukkig, most happy.

4. The Superlative is preceded by het when the highest quality is predicated to an object under certain circumstances, as

De Schelde is het breedst in The river Scheldt is broadest in Zeeland, Zeeland.

Adjectives which do not admit of comparison are those of material ; those denoting the acme of a quality and mathematical qualities; those ending in loos (such as vaderloos, fatherless); adjectives like bloedrood, blood-red, sneeuwwit, snow-white, \&c.; those which have an absolute signification, as rond, vierkant; also geographical adjectives.

Irregular Comparison. - The following form their degrees of comparison irregularly:--

$\begin{array}{lll}\text { goed, good } & \text { beter, better } & \text { best, best } \\ \text { weinig, little } & \text { minder, less } & \text { minst, least } \\ \text { veel, much } & \text { meer, more } & \text { meest, most } \\ \text { livad, bad } & \text { erger, worse } & \text { ergst, worst. }\end{array}$

Some adjectives are used only attributively, as (1) those of material, time, and place, like dagelijksch, bovenste; (2) those denoting the place where someone lives, as Amsterdamsch; (3) those which indicate the manner in which something takes place, as eene mondelinge boodschap. Other adjectives are used only predicately. Such are:Bedacht, beducht. behept, bestand, bijster, kwijt, war's, handgemeen, leed.

\section{The Pronoun. (Het Voornaamwoord.)}

There are seven divisions of Pronouns:-

1. Personal. 4. Interrogative.

2. Possessive.

5. Relative.

6. Indefinite.

7. Determinative.

3. Demonstrative.

\section{Personal Pronouns.}

Singular.

1st per.

2nd per.

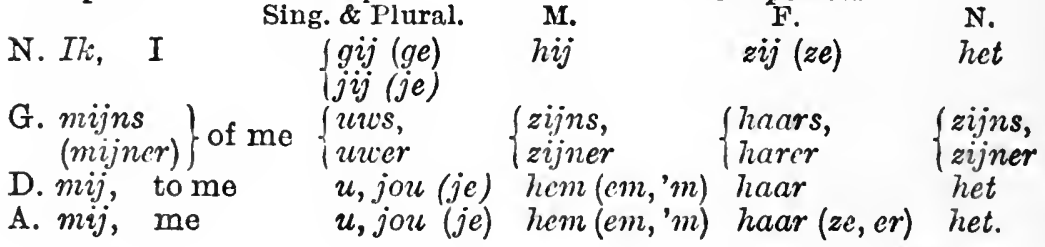

1st per.

N. $w i j(w e)$

$\mathrm{G}$. ons, onzer

D. ons

A. ons

Plural.

M.

$z i j(z e)$

huns, hunner

hun, ze

hen, ze 3rd person.

F. $z i j(z e)$

$\quad z i j(z e)$

haar, harer huns, hunner

haar (er) hun, ze

haar (ze, er) hen, ze. 
-Het" and its use.-The names of inanimate objects in Dutch being often masculine or feminine, the pronoun referring to them must take their gender; therefore the word it cannot always be rendered by het, as

Hebt gij mijn hoed (m.) gezien? Have you seen my hat?

Neen, it heb hem niet gezien. No, I have not seen it.

Hebt gij mijn boek (n.)? Have you my book?

$J a, i k$ heb het. Yes, I have it.

The dative and accusative of het, when governed by prepositions, are always rendered by $e r$, to which the preposition is appended. Het is often abbroviated to ' $t$, as neem ' $t$, take it.

Nоте.-1. In colloquial language $g e, v e, m e$, and $z e$ are used tnstead of $g i j$, $w i j, m i j$, and $z i j$. In conversation $U$ is used for the singular as well as for the plural, as

Wil U mij den weg wijzen? Will you please show me the way?

2. In writing, gij is used in addressing friends; in other cases $U$ (uwo edelheid), with the verb in the 2 nd person plural or 3rd person singular, as $U$ hebt or $U$ heeft.

3. The genitives mijns, ons, uws, zijns, haars, occur in connection with zelfs, or gelijke only, as

Hij en zijn. gelijken, he and persons like himself.

Om haars zelfs wil, for her own sake.

4. To emphasize the personal pronouns, the word zelf (sing.), pl. zelven. is often added, as

Ik zelf heb het gezien. I have seen it myself.

Wij zelven hebben het gehoord, we have heard it onrselves.

5. The Personal prononus are sometimes used instead of the Demonstrative, and often instead of the Correlative prououns. (See p. 79.)

\section{Reflexive and Reciprocal Pronouns.}

The Dative and Accusative of the personal pronouns are used as Reflexive Pronouns, the third person having the Reflexive form $z i c h$, which is used in the dative and accusative only for all genders, singular and plural.

Elkander (elkaar) and malkander (malkaar), each other, are Reciprocal Pronouns, and take $s$ in the Genitive; the Dative and Accusative having the same form as the Nominative, as wij sullen elkander schrijien, we shall write to each other.

\section{Possessive Pronouns.}

The Possessive Pronouns are: mijn, my; ons, our; $u w$, thy or your; zijn, his or its; hun, their; haar, her or their.

They are used with or without a noun, and declined in the former case like mijn (except ons, which has the form onze in the nominative singular masculine).

Singular.

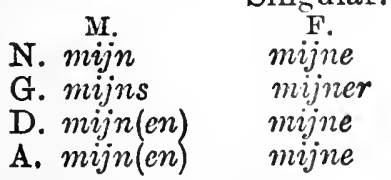

$\begin{array}{lc}\text { N. } & \begin{array}{c}\text { Plural } \\ \text { (all genders). }\end{array} \\ \text { mijn } & \text { mijne } \\ \text { mijns } & \text { mijner } \\ \text { mijn(en) } & \text { mijnen } \\ \text { mijn } & \text { mijne. }\end{array}$

NoTE.-Instead of mijne and mijnen, mijn is often used, as $I k$ roep mijn broeder, I call my brother; aud mijne is commonly pronounced as mijn. 
When used substantively and preceded by an article, they are declined as follows:-
N. de mijne
Singular.
G. des mijnen
F.
de mijne
D. den mijnen
der mijne
de mijne
A. den mijnen
de mijne
N.
het mijne
van het mijne
het mijne
het mijne
Plural
(all genders).
de mijne
der mijne
den mijnen
de mijne.

NotE.-1. Mijnen, zijnen, uwen are used, when no noun precedes them, in

the plural of relations, friends, \&c., as

Groet de uwen, remember me to your people.

2. Often in Dutch the Definite Article is used when in English the possessive pronoun is nsed, as

De oude man schudde het hoofd, the old man shook his head.

\section{Demonstrative Pronouns.}

Demonstrative pronouns may be used substantively or adjectively. In the substantive form they are of the strong declension.

The articles dit, deze, this one, die or dat, gene, that, dezelfde, diezelfde, the same, are used substantively and adjectively. Zelve, self, is used adjectively only. Deze and die are inflected like the article de, dat like het.

Deze and die are declined as follows:-

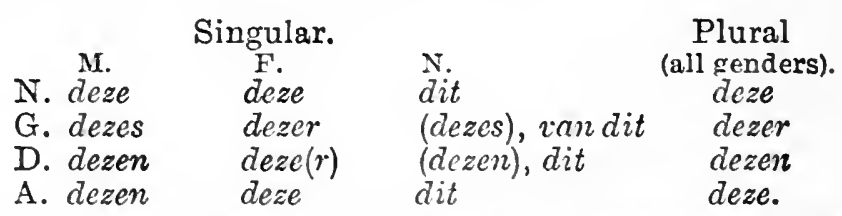

Note.-The genitive of deze, used substantively, is always paraphrased.

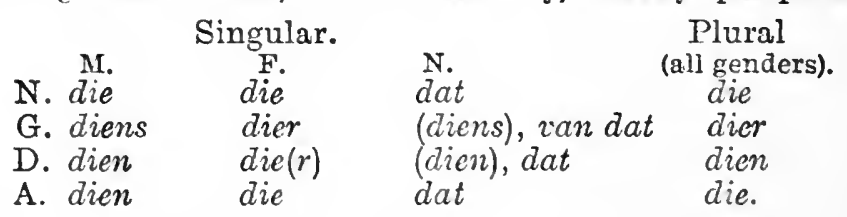

Gene is inflected like deze, but has no singular form for the Nominative, for which gindsch is used:-gindsch huis, that house (yonder); gene huizen, those houses (yonder). The Genitive is paraphrased by van, the Dative by aan, which is also used with dit and dat, as van dien, van die, van dat, and van die, instead of the Genitives diens, dier, \&c.

Dezelfde, hetzclfde, diezelfde, datzelfde, used adjectively, are inflected as an adjective preceded by an article. When used substantively, they are declined like an adjective used substantively:-
Singular.
N. dezelfde
G. deszelfden F.
D. denzelfden dezelfde derzelfde dezelfde
A. denzelfden dezelfde
Plural
N.

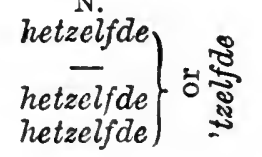
(all genders).
dezelfde derzelfde denzelfden dezelfde. 
Degene has no Genitive form for the neuter. For the Numinative singular datgene is used. In the other eases it is declined like an adjective used substantively.

Zelve is placed after substantives and personal pronouns to strengthen their signification. The word zelf may be used invariably, as ik zelf, I myself. When it is declined, zelf is inflected as on adjective used substantively, but it has no Genitive form.

$\begin{array}{lccc} & \text { Singular. } & \text { Plural } \\ \text { M. zelf } & \text { F. } & \text { N. } & \text { (all genders). } \\ \text { Nelve } & \text { zelve } & \text { zelve } \\ \text { D. zelven } & \text { zelve } & \text { zelve } & \text { zelven } \\ \text { A. zelven } & \text { zelve } & \text { zelve } & \text { zelve. }\end{array}$

Sometimes a personal pronoun is used instead of a demonstrative, as

Kent gij dien officier? Do you know that officer?

Neen hem niet, wel den andere. No; not that one, but the other.

Correlative Pronouns. - The Demonstrative Pronouns degene, \&c., when connected with a Relative Pronoun, are termed CoRretative Pronouns. They are: degene, die or die, welke, wie, dat, wat, datgene, hetgeen, and the Personal Pronouns hij, zij.

\section{Interrogative Pronouns.}

There are four Interrogative Pronouns :-

Wie? (m. \& f.) who? applies to persons, and is always used substantively.

Wat? (n.) what? is used substantively and adjectively, and applies to inanimate objects. It is only used in the Nominative and Accusative singular. In the other cases it is paraphrased by a preposition with the adverb waar; i.e., instead of van wat, aan wat, in wat, \&c., waarvan, waaraan, waarin. \&c., are used.

Welk(e)?-which ? applies to persons and objects, used adjectively, and declined like a Possessive Pronoun. The genitive is paraphrased by van (see under). The strong undeclined form Welk? is used in the nominative and accusative singular of the masculine when one asks after a person in his dignity or profession. The weak form welke? (Acc., welken?) asks after the name of a person.

Hoedanige?-what kind of? This is somewhat obsolete, and the combination of wat voor een? (een only being declined) is frequently used, as Wat voor een man is hij? - what sort of man is he?-insteal of Hoedanig een man is hij?

The Interrogatives are declined as follows:-

$$
\text { Singular. Plural. }
$$

$\begin{array}{lllll} & \text { M. } & \text { F. (both genders) } & \text { N. } \\ \text { N. Who? } & \text { wie? wie? } & \text { wie? } & \text { What? wat? } \\ \text { G. of whom? } & \text { wiens? (wier?) wier? } & \text { of what? waarvan? } \\ \text { D. whom? } & \text { wien? wie? } & \text { wien? } & \text { to what? waaraan? } \\ \text { A. whom? } & \text { wien? wie? } & \text { wie? } & \text { what? wat? }\end{array}$




\section{Singular.}

M.
N. Which? welk(e)?

\section{Examples:-}

Welken (m.) man bedoelt gij? Which man do you mean?

Welke (n.) boeken hebt gij gekocht? Which books did you buy?

The English interrogation, "What is your name?" is sometimes rendered in Dutch by Hoe heet gij?

Instead of welk, wat voor een (or simply wat) can be used:Wat man zou dat doen?

\section{Relative Pronouns.}

The Relative Pronouns are die, who; dat, that; welk, which; and must agree with their antecedents in gender, number, and person. Die is declined thus :-

$\begin{array}{lllll} & & \text { Singular. } & & \text { Plural. } \\ \text { N. Who } & \text { die } & \text { F. } & \text { N. } & \text { (all genders). } \\ \text { G. of whom wiens } & \text { wier } & \text { dat waarvan } & \text { wie } \\ \text { D. to whom } & \text { wien } & \text { wie } & \text { wat } & \text { wien } \\ \text { A. whom } & \text { dien,wien } & \text { die, wie } & \text { dat } & \text { die, wie. }\end{array}$

In the accusative wien and wie are used with a preposition only, in all other cases die and dat, as

De heer met wien, de dame The gentleman with whom, the met wie it reisde. lady with whom I travelled.

In speaking of a woman, welke is sometimes used instead of die, as Het is die, welke ik zocht, it is she whom I was looking for.

The genitive masculine welks seldom refers to persons. The nominative and accusative is welk when used adjectively.

The Relative Pronoun can neyer be omitted in Dutch, as is done in English.

De vriendin die ik liefleb. The friend (whom) I love.

\section{Indefinite Pronouns.}

The principal Indefinite Pronouns are:-

*men, one, they, people.

* iemand (takes $s$ in the genitive), someone, somebody.

* niemand (takes $s$ in the genitive), no one, nobody.

*iets, something, anything; *niets, nothing; een, one ; geen, none; zeker, certain (only used adjectivally);

*een iegelijk, iedereen, elkeen or een ieder, everybody, everyone.

* elkander or malkander, each other, one another (may take s in the genitive). 
The following are sometimes used as nouns, when referring only to persons, in which case they take $\mathbf{n}$ in the nominative, dative, and accusative cases :-

alle, all; sommige, some; eenige, some ;

vele, many; weinige, fow; ettelijke, sundry.

Notr. - 1 , Pronouns marked " may only be used substantively.

2. Ieder, elk, everyone, everybody, can be used substantively or adjectivally.

3. Tedereen and elkeen are invariable.

4. Een iegelijk, een ieder, ieder, and elk have no plural, and in the Genitive singular take $s$ when used substantively.

\section{Numerals. (Telwoorden.)}

The Numerals, most of which are a special kind of adjective, are divided into several classes.

Cardinal Numbers (see page 54) denote simple numbers, as een, twee, \&c.

Norg.-1. Een and the negation geen, when joined to a noun, are declined like the Indefinite Article.

2. Beide, both, is declined like the Definite Article; if preceded by a defining word it is declined like an Adjective.

3. Millioen, billioen, trillioen, milliard are Neuter substantives, plural with en.

4. Honderd and duizend, when not preceded or followed by a numeral, are also substantives; plural $e n$.

5. The real Cardinal Numbers are indeclinable, except when used substantively, as twee vieren, two fours.

6. Honderd, if preceded by another numeral, is written with the preceding number in one word, as tweehonderd; but not duizend, as vier duizend.

Ordinal Numbers (see page 55) denote numbers as forming parts of a series, and are formed from the Cardinals by adding de from 2-19, and ste from 20 upwards.

Exceptions: Eerste, achtste. Instead of driede, derde is used.

The Ordinals are declined like adjectives.

NoTe.-1. When referring to two persons or objects, "the former" and "the latter " are translated by de eerste and de laatste, respectively, or deze and gene.

2. The DATE is translated thns: De twee en twintigste April, the 22nd of April.

3. Distinctrogs are formed from the Ordinals by prefixing the word ten, as ten derde, thirdly.

4. MULTIPLicatives are formed by adding voud to the Cardinal numbers, as tweevoud, twofold, and are used substantively only. To form an adjective or an adverb from these words, $i g$ must be added, as tweevoudig.

5. NUMBERS OF REPETITION are adrerbs which are formed by adding maal, keer, or werf to the Cardinal numbers, as eenmal, once; tweekeer, twice; driewerf, thrice, \&c.

6. Fractional NUMBERs are formed by the suffix de, EXOEPT half, half (one-half, een half), as een derde, one-third; een vierde, one-fourth. When half is used as an adjective, it is placed after the article, as een half uur, half-an-hour. 


\section{Xerbs.* (Werkwoorden.)}

\section{Auxiliary Verbs. .}

The Dutch language has four Auxiliary Verbs:$Z i j n$ or Wezen, to be. Hebben, to have.

Worden, to become.

Zullen, will or shall.

Infinitive Present.

$Z_{i j n}$, Wezen, to be Worden, to be (become) Hebben, to have.

\section{Perfect.}

geweest zijn,

to have been

zijnde, being

geweest, been

\author{
geworden zijn,
}

to have been

Present Participle.

wordende, being

Past Participle.

geworden, been

\section{Indicative Mood. $\uparrow$}

Present.

ik ben, I am

hij is

wij zijn

gij $z i j t$

$z i j z i j n$

th was, I was

hij was

wij waren

gij waart

zij waren

$i k$ ben geweest,

I have been hij is

wij zijn

gij zijt geweest

zij zijn

ik was geweest,

I had been

hij was

wij waren

gij waart

zij waren ik word, I am

hij wordt

wij worden

gij wordt

zij worden

Imperfect.

ik werd, I was

hij werd

wij werden

gij werdt

zij werden

Past or Perfect Definite.

$i k$ ben geworden,

I have been

$\left.\begin{array}{l}\text { hij is } \\ \text { wij } z i j n \\ \text { gij zijt } \\ z i j \text { zijn }\end{array}\right\}$ geworden

Pluperfect.

ik was geworden,

I had been

hij was

wij waren

gij waart

zij waren gehad hebben, to have had.

hebbende, having.

gehad, had.

it heb, I have

hij heeft

wij hebben

gij hebt

zij hebben.

ik had, I had

hij had

wij hadden

gij hadt

zij hadden.

it heb gehad,

I have had

hij heeft

wij hebben

$\left.\begin{array}{l}\text { wij hebben } \\ \text { gij hebt } \\ \text { zij hebben }\end{array}\right\}$ gehad.

ite had gehad,

I had had

hij had

wij hadden

$\left.\begin{array}{l}\text { wij hadden } \\ \text { gij hadt } \\ \text { zij hadden }\end{array}\right\}$ gehad.

* For list of Verbs, see pp. 60-64.

t In conjugating the verbs, the 2nd pers. sing. is omitted, except in the Imperative, as nnnocessary. 
ite zal zijn. I shall be

hij $z a l$

wij zullen

gij zult zijn

zij zullen

ik zal genveest zijn, I shall have been

ik zou(de) zijn,

I should be

hij zou (de)

wij zouden

$\left.\begin{array}{l}\text { wij zoudt } \\ \text { zij zouden }\end{array}\right\}$ zijn

Future Indefinite.

ite zal worden,

I shall be

hij zal

wij zullen worden

$\left.\begin{array}{l}\text { gij zult } \\ \text { zij zullen }\end{array}\right\}$

Future Perfect.

ik zal geworden zijn, ik zal gehad hebben,

I shall have been I shall have had.

Present Conditional.

ik zoiu worden,

I should be

hij zou wij zouden $_{\text {worden }}$

gij zoudt

zij zouden

Past Conditional.

ik zoud(e) geweest zijn, ik zou geworden zijn, ik zou(de) gehadhebben,

I should have been I should have been I should have had

Imperative Mood.

Sing. wees! be (thou)! word! be (thou)! Plur. weest $l$ be (you)! wordt! be (you)! hebt ! have (you)!

Subjunctive Mood.

Present.

$i k z i j$, that I may be $i k$ worde, that I may $i k$ hebbe, that I may

hij $z i j$

wij zijn

gij zijt

$z i j z i j n$

ik ware,

that I might be

hij ware

wij waren

gij waret

zij waren hij worde

wij worden

gij wordet

zij worden

Imperfect.

it werde, that I might be

hij werde

wij werden

gij werdet

zij werden it zoul(de) hebben,

I should have

hij zon

wij zouden

gij zoudt

zij zouden hebben.

I shall have

hij zal

gij zult hebben.

zij zullen 
Pluperfect.

itk ware geweest, that

I might have been

$\left.\begin{array}{l}\text { hij ware } \\ \text { wij waren } \\ \text { gij waret } \\ \text { zij waren }\end{array}\right\}$ geweest ik ware geworden, that

I might have been hij ware

wij waren

gij waret $\}$ geworden it hadde gehad, that

I might have had

hij hadde

wij hadden

$\left.\begin{array}{l}\text { wij hadden } \\ \text { zij haddet } \\ \text { zidden }\end{array}\right\}$ gehad.

Zullen, shall, will.

Infinitive Present. zullen.

Indicative Present.

if zal, I shall

hij zal, wij zulien

gij zult

zij zullen
Present Participle. zullende.

Subjunc. Imperf.

itc zoude, I should

hij zoude

wij zouden

gij zoudet

zij zouden.

The other tenses are wanting.

The Dutch past participle must always be placed at the end in sentences with compound tenses:-Hij is koning geweest, he has been king.

The auxiliary verb of compound tenses comes last when, in dependent clauses, the verb is placed at the end of the clause:-lk geloof, dat hij hier geweest is, I believe he has been here.

In compound tenses the participle or adjective must be placed directly after the verb, as Hij is gestraft geworden, he has been punished.

The intransitive verb worden, to become, is conjugated in the same manner as worden, to be.

\section{CONJUGATION OF VERBS.}

\section{Dutch Regular Verbs are divided into Weak and Strong.}

Past Participle.-Weak Verbs have no vowel change, their past participle being formed by adding $d$ or $t$ to the root, and that of Strong Verbs by adding $e n$ to the root.

The Infinitixe of all verbs is formed by adding $e n$ to the root.

The 1st person of Present Indicative is the stem of the verb.

NoTE. -1 . When the root ends in a double consonant, both consonants are written before $e$ alone; in all other cases only one is written.

2. The 1st person singular has the same form as the root.

3. The 2nd person in all tenses of a verb always ends in $t$.

4. The 1st and 3rd person plural always end in $e n$.

5. The 3rd person Present Indicative always ends in $t$. Exceptions:hij kan, he can; hij zal, he will; he will, he wants; hij is, he is; hij mag, he may.

6. The past participle takes ge, except with verbs which begin with the prefexes $b e, g e, e r$, her, ont, and ver, when they are inseparably compounded with an adverb. The root of the verb then has the accent.

\section{Weak Verbs.}

All weak verbs form the Imperfect Indicative by adding $d_{\theta}$ to the root, and the Past Participle by adding $d$. 
When the root ends in $c h, f, k, p, t, s, s c h, d$ and de change into $t$ and te.

Should the root end in $d$ or $t$, no extra $d$ or $t$ is added in the past participle:-dooden (to kill), gedond-but doodde: praten (to talk), gevraat-but praatte.

When the final consonant of the root is $s$ or $f$, it changes into $\mathrm{z}$ or $\mathrm{X}$ in the infinitive, as:

vreezen, to fear; root vrees (not vreez).

leven, to live; " leef (not leev).

The 3rd person singular of the Imperfect Indicative has the same form as the 1st.

The 1st and 3rd person plural are formed by adding den or ten to the root.

\section{ANTwOoRden, to answer.}

Infinitive Present.

Antwoorden, to answer. geantwoord hebben, to have answered.

Present Participle.

antwoordende, answering.

Past Participle.

geantwoord, answered.

Indicative Mood.

Present. ik antwoord, I answer

hij antwoordt wij antwoorden gij antwoordt

sij antwoorden.

Pluperfect.

Pluperfect.
ik had geantwoord,
ik zal antwoorden I had answered.
Imperfect. ite antwoordde, I answered hij antwoordde wij antwoordden gij antwoorddet zij antwoordden. ik zal antwoorden, I shall answer.
Perfect. ik heb geantwoord, I have answered hij heeft wij hebben gij hebt zij hebben geantwoord.

Future Perfect. ik zal geantwoord hebben, I shall have answered.
Pres. Conditional. ik zou(de) antwoorden, I should answer.
Past Conditional. ik zoude geantwoord hebben, I should have answered.

Subjunctive Mood.

Present.

ite antwoorde, that I may answer hij antwoorde wij antwoorden gij antwoord $(e) t$ zij antwoorden.

\section{Perfect.}

ik hebbe geantwoord, that I may have answered.

Pres. Conditional.

ik zoude antwoorden, that I should answer.
Imperfect. ik antwoordde, that I might answer hij antwoordde wij antwoordden gij antwoorddet zij antwoorden.

\section{Pluperfect.}

ik hadde geantwoord, that I might have answered.

Past Conditional.

ik zoude geantwoord hebben, that I should have answered.

Imperative Mood.

Sing., antwoord! answer (thou)! Pl., antwoordt ! answer (you)! 
The Negative form is like that of English auxiliary verbs :ik antwoord niet, I do not answer.

The Interrogative form corresponds also to that of English auxiliaries:-
antwoord ik?
do $I$ answer?

The Negative-Interrogative form:-

antwoord ik niet? do I not answer?

\section{Strong Verbs.}

A strong verb forms its past tense by a change in the radical vowel without any termination, and the past participle by the termination en and sometimes by a change of the radical vowel, as geven, to give; gaf, gave; gegeven, given.

stelen, to steal; stal, stolen; gestolen, stolen.

As will be seen, the personal endings in the strong conjugation are the same as those of the weak.

In conjugating a strong verb, the Infinitive, the singular of the Imperfect Indicative, and the Past Participle must be known. All other tenses are formed like those of a weak verb, with the exception of the Imperfect Subjunctive, which is formed from the plural of the Imperfect Indicative, as wij gaven, ik (hij) gave.

The 3rd person plural Imperfect Subjunctive and Imperfect Indicative are alike.

Geven, to give.

\section{Indicative Mood.}

Present.

ik geef, I give

hij geeft

wij geien

gij geeft

zij geven.

Pluperfect.

it had gegeven,

I had given.
Imperfect.

it: gaf, I gave

hijgaf

wij gaven

gij gaaft

zij gaven.

Future Indefinite. ik zal geven, I sball give.
Pres. Conditional. ik zou (de) geven,

I should give.
Perfect.

it heb gegeven, I have hij heeft ) [given wij hebben $\left.\begin{array}{l}\text { gij hebt } \\ \text { zij hebben }\end{array}\right\}$ gegeven.

Future Perfect. ik zal gegeven hebben, I shall have given.
Past Conditional.

ik zou(de) gegeven hebben,

I should have given.

Subjunctive Mood.

\section{Present.}

ik geve, (that) I may give.

Perfect.

it hebbe gegeven,

(that) I may have given.

Pres. Conditional.

it zoude geven,

(that) I should give.

Imperfect.

ik gave, (that) I might give.

ik hadde gegeren,

(that) I might have gịven.

Past Conditional.

it zoude gegeven hebber.

(that) I should have given.

Imperative Mood.

Sing., geef ! give (thou) !

Pl., geeft / give (you) ! 


\section{Classes of Strong Verbs.}

The strong verbs may be divided into seven classes, according to the vowel change. When these changes are known the conjugation is like that of a weak verb, and presents no difficulty.

\section{1st Class.}

The verbs of the first class have in the present tense $e$ or $i$, followed by $l, m, n$, or $r$ with another consonant; this second consonant may be the same as the first, or may be different. In the singular and plural of the Imperfect and in the Past Participle they have short $o$. Ex. :-

\begin{tabular}{cccccc} 
Infinitive. & \multicolumn{2}{c}{ Imperfect. } & & & Past Part. \\
beginnen, to begin & $\ldots$ & begon & $\ldots$ & $\ldots$ & begonnen \\
bergen, to hide $\ldots$ & $\ldots$ & borg & $\ldots$ & $\ldots$ & geborgen.
\end{tabular}

N.B.-The verbs helpen (to help), bederven (to spoil), sterven (to die), werpen (to throw), werven (to recruit), and zwerven (to wander) change $o$ into ie in the Imperfect; thus-

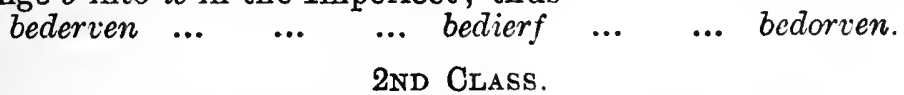

The verbs of this class have in the present tense a long e, followed by a liquid consonant or by $k$. The singular of the Imperfect has a short $a$, the plural a long $a$, the Past Participle a long o. Ex. :-

bevelen, to order ... $\quad \ldots$ beval $\quad . . \quad \ldots$ bevolen.

The following deviate from the vowel-change in this class:-

$\begin{array}{lllllll}\text { komen, to come } & \ldots & \ldots & \text { kwam } & \ldots & \ldots & \text { gekomen } \\ \text { zweren, to swear } & \ldots & \ldots & \text { zwoor } & \ldots & \ldots & \text { gezworen } \\ \text { scheren, to shave } & \ldots & \ldots & \text { schoor } & \ldots & \ldots & \text { geschoren } .\end{array}$

3rd Class.

In this class the verbs have in the present tense and in the Past Participle a long $e$, followed by a single consonant (except $l, m, n$, $r$, and $k_{i}$, in the singular of the Imperfect a short $a$, and in the plural of the Imperfect a long $a$. Ex.:-
lezen, to read
-. las
... $\quad \ldots$ gelezen
geven, to give $\quad \ldots \quad \quad \ldots \quad$ gaf $\quad \ldots \quad \ldots$ gegeven.

N.B.-Bidden (to pray), liggen (to lie), and zitten (to sit) have $i$ instead of $e$ in the Infinitive Mood and in the present tense.

\section{TH Class.}

Verbs of this class have in the present tense $i j$, in the Imperfect $e e$, and in the Past Participle e. Ex.:-

$$
\begin{aligned}
& \text { belijden, to confess } \quad \ldots \text { beleed } \quad \ldots \quad \ldots \text { beleden } \\
& \text { bezwijken, to succumb } \ldots \text { bezweek } \ldots \text {... } \\
& \text { bijten, to bite } \quad . . \quad \text {... beet } \quad . . \quad \text {... gebeten. }
\end{aligned}
$$

(Aan)tijgen, to impute; bezwijmen, to swoon; grijnen, to fret; hijgen, to pant; krijschen, to shriek; stijven, to encourage; prijzen, to state a price, are weak. Krijgen, to get; stijuen, to make stiff (e.g., linen); and prijzen, to praise, are strong. 


\section{TH Crass.}

Verbs in this class have in the present tense ie or $u i$, in the singular of the Imperfect 00 , in the plural of the Imperfect and in the Past Participle o. Ex. :-

$$
\begin{array}{lllllll}
\text { bedriegen, to cheat } & \ldots & \text { bedroog } & \ldots & \ldots & \text { bedrogen } \\
\text { bieden, to offer } & \ldots & \ldots & \text { bood } & \ldots & \ldots & \text { geboden. } \\
\text { buigen, to bend } & \ldots & \ldots & \text { boog } & \ldots & \ldots & \text { gebogen. }
\end{array}
$$

Schuilen, to hide, and zieden, to boil, are weak or strong. Klieven, to cleave, is weak; kluiven, to gnaw, is strong. Jagen, to hunt, and vragen, to ask, have a weak and a strong Imperfect, but a weak Past Participle.

\section{TH Class.}

The verbs of this class have in the present tense and in the Past Participle a long $a$, and in the Imperfect singular and plural oe. Ex. :-

$$
\begin{array}{lllllll}
\text { dragen, to carry } & \ldots & \ldots & \text { droeg } & \ldots & \ldots & \text { gedragen } \\
\text { graven, to dig } & \ldots & \ldots & \text { groef } & \ldots & \ldots & \text { gegraven } .
\end{array}
$$

Lachen, to laugh, has a weak Imperfect, as also laden, to load (Imp. laadde), and malen, to grind (Imp. maalde).

\section{TH Class.}

In this class the verbs have the same vowel in the present tense

\begin{tabular}{|c|c|c|c|c|c|c|}
\hline reken, to & & & brak & $\ldots$ & $\ldots$ & aebroken \\
\hline & & & & $\cdots$ & $\ldots$ & \\
\hline & $\cdots$ & $\cdots$ & dacht & & $\ldots$ & ged \\
\hline to dc & $\cdots$ & $\ldots$ & deed, ded & len* & $\ldots$ & gec \\
\hline aink & $\cdot \cdot$ & $\cdots$ & doc & $\ddot{d} \ddot{d r e t}$ & $\cdots$ & $g$ \\
\hline$\theta$ & ... & $\begin{array}{l}\cdots \\
\cdots\end{array}$ & ging & ... & $\cdots$ & gegaan \\
\hline ve & $\ldots$ & $\ldots$ & had & $\ldots$ & $\ldots$ & \\
\hline & $\cdots$ & $\cdots$ & kocht & & $\ldots$ & gekocht \\
\hline iur & $\cdots$ & $\ldots$ & kon, kiona & den & $\ldots$ & gekund \\
\hline$e n, \mathrm{~m}$ & $\ldots$ & $\ldots$ & moest & $\ldots$ & $\ldots$ & gemoeter \\
\hline allor & wed & $\cdots$ & mocht & $\cdots$ & $\ldots$ & gemoog \\
\hline יטבם & & & placht & & $\ldots$ & - \\
\hline $\operatorname{taan}$ & $\ldots$ & $\ldots$ & stond & .. & $\cdots$ & gestaan \\
\hline & $\ldots$ & $\ldots$ & wist & $\ldots$ & $\ldots$ & geweten \\
\hline & $\ldots$ & ... & wilde & wou & & gewild \\
\hline & $\cdot$ & ... & $z a$ & $\ldots$ & $\ldots$ & gezien \\
\hline & $\cdots$ & & $z o c h t$ & $\ldots$ & $\cdots$ & $\operatorname{coh} t$ \\
\hline & & . & $s, ?$ & ren & $\cdots$ & eweest \\
\hline & & & & & & \\
\hline
\end{tabular}
and in the Past Participle. In the Imperfect, a short or long : $(i, i e)$. Ex. :-

$$
\begin{array}{lllllll}
\text { blazen, to blow } & \ldots & \ldots & \text { blies } & \ldots & \ldots & \text { geblazen } \\
\text { braden, to roast } & \ldots & \ldots & \text { braadde } & \ldots & \ldots & \text { gebraden } .
\end{array}
$$

\section{Irregular Verbs.}

- Separate forms for singular and plural. See other instances below. 


\section{Compound Verbs.}

Verbs are compounded with certain prefixes, which are either separable or inseparable from the verbs.

Inseparable when the verb is compounded with a substantive, another verb (in which case the first verb drops the $n$ ), an adjective, or one of the adverbs door, mis, om, onder, over, voor, which are not accented and do not take ge in the Past Participle. Ex.:-

liefkoozen, to caress-Past Participle, geliefkoosd;

handhaven, to maintain-gehandhaafd.

These compounds are all weak, even if the verb itself should be strong and take ge in the Past Participle.

Verbs compounded with the adjective vol (full) are inseparable, and do not take ge in the Past Participle, as Het is volbracht, it is finished.

Separable applies to compound verbs of which each part may be used in its original signification. These verbs are compounded with a substantive, an adjective, or an adverb. The last have the accent on the adverb. The verb has its proper conjugation, and the prefix ge is placed before the verb, as :-

paardrijden, to ride on horseback;

ik rijd paard; ik reed paard; ik heb paardgereden.

Verbs compounded with the inseparable prefixes be, ge, er, her, ont, and ver do not take ge in the Past Participle, as :-
behouden, to retain-Past Part., behouden;
gelooven, to believe-geloofd;
erkennen, to acknowledge-erkend;
herinneren, to remember-herinnerd;
onthouden, to remember-onthouden;
verbranden, to burn-verbrand.

Compound verbs with the prefixes door, mis, om, onder, over, voor, and weder are either separable or inseparable.

(a) When these prefixes, and the simple verbs united with them, are used in their full and original meaning, the compound verbs are separable; in this case the prefix takes the accent.

(b) When these prefixes have lost their original meaning, the verbs are inseparable and the verb takes the accent.

Thus:-De reiziger heeft den geheelen nacht door'-gereisd.

The traveller has travelled all night.

De reiziger heeft het geheele continent door-reisd'.

The traveller has travelled through the whole country.

When the clause is a principal sentence and the verb is in a simple tense the prefix is placed at the end, as Ga met mij uit, go out with me. When beginning with a Relative Pronoun or a subordinating conjunction, the prefix keeps its place before the verb, the verb being placed at the end, as:-

Ik wou wilde, dat hij terugkwam. I wish he would return. 


\section{Reflective Verbs.}

Reflective Verbs are frequent in Dutch, and besides their subject they always take a Reflective Pronoun of the same person and number as their subject, the pronoun usually being in the Accusative, as Ik herinner mij, I remember.

NoTE.-1. Some verbs take the Reflective Prononn in the Dative, as Hij geeft zich moeite, he gives himself trouble.

2. Some Reflective Verbs are rendered in English by a Passive Verb, as zich schamen over, to be ashamed of ; zich vergissen, to be mistaken.

3. Some rerbs reflectixe in Dutch are not so in English, as zich aanmatigen, to assume; zich baden, to bathe.

All Reflective Verbs are conjugated with the assistance of the auxiliary hebben, as $I k$ heb mij herinnerd, I have remembered.

\section{Impersonal Verbs.}

Impersonal Verbs are conjugated only in the 3rd person singular with the neuter pronoun het.

There are some verbs - as heugen, to remember, spijten, to be sorry-which only seem to be impersonal, but which really are personal. When one says Het spijt mij, dat gij ziek zijt, the real subject is dat gij ziek zijt, and het is only the substituting subject.

\section{GENERAL NOTES ON THE VERBS.}

A verb always agrees with its subject in number and person.

The conjugation in English by the Present Participle with the Auxiliary Verb to be is not used in Dutch; hence "I read," "I am reading." are rendered in Dutch by It lees.

In like manner, the conjugation which, in all its tenses, represents a future action does not exist in Dutch; so that such a phrase as "I am about to write," is translated Ik ga juist schrijven.

The Gerund (as erring, reading, writing) is rendered in Dutch by the Infinitive used as a noun, as Dwalen is menschelijk, erring is human.

With Imperatives, and in negative interrogative sentences, the English auxiliary verb to do is not translated in Dutch. It can be rendered by toch in Imperatives, as

$$
\text { Schrijf mij toch. Do write to me. }
$$

The use of the Infinitive differs in many respects from the English. If a verb is named, it does not take the particle te (to) as in English, but is simply rendered in Dutch thas: gaan, to go; lezen, to read, \&c.

In some phrases the Infinitive takes the place of the Present Participle in English, as Ik zag hem loopen, I saw him running.

The particle te is used with the Infinitive when a purpose or intention is indicated, and strengthened by the addition of om, as

De mensch leeft niet om te eten, maar hij eet om te leven.

Man does not live to eat, bat he eats to live.

$T e$ is also used when the Infinitive expresses the object to which an action, \&c., is directed, as Ik wensch ham te zien, I wish to see him. 


\section{Adxerbs. (Bijwoorden.)}

Adverbs are formed by adding lijk, lijks, lings, e, ens, $s$, jes, pjes, tjes, kens, gewijze, waarts to a noun or adjective, as

$\begin{array}{ll}\text { eigen, proper, } & \text { eigenlijk, properly; } \\ \text { dag, day, } & \text { dagelijks, daily; } \\ \text { kort, short, } & \text { kortelings, shortly; } \\ \text { ver, far, } & \text { op verre na, by far; } \\ \text { minst, least, } & \text { minstens, at least; } \\ \text { recht, right, } & \text { rechts, to the right, } \\ \text { zoet, soft, } & \text { zoetjes, softly; } \\ \text { warm, warm, } & \text { warmpjes, warmly; } \\ \text { stil, silent, } & \text { stilletjes, silently; } \\ \text { zacht, soft, } & \text { zachtiens, softly. } \\ \text { trap, degree, } & \text { trapsgewijze, by degrees. }\end{array}$

Some adverbs, like adjectives, admit of comparison (see page 75), as Hij schrift goed, zijn zuster beter, doch zijn broeder het best, he writes well, his sister better, but bis brother best.

Some words ending in lijk are adjectives, and are used as adverbs without changing their form, such as eerlijk, honest; heerlijk, delightful; weekelijk, delicate, \&c. Words ending in lijks are adverbs, those ending in $l i j k s c h$ are adjectives.

Several adverbs are formed from nouns by adding $s$, as links, on the left; daags, by day.

Adverbs of manner or of time are always placed after the verb, as Hij leest nooit couranten, he never reads newspapers.

Adverbs of place are :-

af, downwards

ergens, somewhere

ginder, ginds,

there, thither

heen, henen, away heen en weer, to and fro

links, to the left na, nabij, near op, upwards thuis, at home ver far, far off waar, where wijd en zijd, far and wide.

Adverbs of time are;aanstonds, directly altijd, altoos, steeds, always dadebijk) terstond immediately doorgaans, generally

gedurig, continually lang, long ooit, ever voorheen
vroeger formerly.

(For list see pp. 64-68.)

\section{Conjunctions. (Voegwoorden.)}

Conjunctions have great influence upon the position of the verb. They are divided into three classes: Co-ordinate, Subordinate, ADVERBIAL.

(a) Co-ordinate join words or sentences which are independent of each other; they are en, and; doch, but; \&c.

The following do not alter the construction: $-E n$, and ; echter, but, however; maar, but; of, or; want, for; zoowel...als, both...and.

(b) Subordinate connect clauses and sentences, one of which is dependent on the other. SIMPLI as well as Compound subordinate conjunctions relegate the verb to the end of the clause. 
When the first clanse of a compound sentence begins with a simple subordinate conjunction, then the principal second clause begins with the verb, and the subject follows it, as Voordat de slag begon, zongen de soldaten vroolijk. Before the battle began the soldiers sang merrily.

Simple SUbordinates are:-

aangezien, daar, as, since ; als, as, when; als of, as if ; dat, that; eer, voordat, before; hoe, how ; \&c., \&c.

CoRrelative Subordinates are:-

aangenomen, gesteld, or verondersteld (dat), supposing (that); al...nog, even if; als...maar, provided (that);

bshalve...dat, besides; om...te, in order to; opdat...niet, lest; zonder...dat, without; zoo...dat, so that; \&c., \&c.

(c) Adyerbial require the subject to follow the verb, instead of preceding it, as Buitendien had hij vele vrienden, besides he had many friends.

The following adverbial conjunctions are treated like adverbs, and require inversion of the clause:-

$\begin{array}{ll}\text { behalve, besides } & \text { dus, thus } \\ \text { bijgevolg, consequently } & \text { echter, evenwel, however } \\ \text { bovendien, buitendien, } & \text { niet alleen...maar, not only } \\ \quad \text { besides, moreover } & \text { niet slechts...maar, but also } \\ \text { daarom, therefore } & \text { noch...noch, neither...nor } \\ \text { dientengevolge, accordingly } & \text { of...of, either...or. }\end{array}$

NотE.-All interrogative adverbs have in indirect questions the force of Relative Conjunctions; the rerb therefore requires to be placed at the end of the clause.

(For list see pp. 64-68.)

\section{Prepositions. (Voorzetsels.)}

All Prepositions govern the Accusative, with the exception of $t e$, which, in some ancient expressions, takes the Dative after it, as Te goeder trouw, in good faith; te elfder ure, very late, almost too late; ten huize van, in the house of. In such cases also some other Prepositions govern the Dative, as In den beginne, in the beginning; van goeden huize, of gentle birth; bij monde van, by the words of ; onder cede, upon oath; met dien verstande, under conditions; uit hoofde van, on account of. Nearly all Prepositions are used in the same way as adverbs.

(For Prepositions in general use see pp. 64-68.)

\section{Interjections. (Tusschenuerpsels.)}

Interjections express:-

Imitations of Sounds, such as Boem! plof! plomp! krik! krak! paf! paf! \&c.

JoY and SURPRISE: ha! heisa! hoezee! vivat! he! hei! holla! GRIEF and PAIN : ach! ai, ou! och! helaas! o wee!

Dолвт: ei! ei ei! wel! hm!

ABHORRENCE : $a b a ! b a !$ foei!

NOTr.-Expressions such as lieve hemell (good gracions!), goede hemell (good heavens l), \&c., are sometimes used as interjections, although they are whole sentences or substantives in the vocative. 


\section{USEFUL AND NECESSARY IDIOMATIC EXPRESSIONS AND PHRASES.}

\section{(Eigenaardige Uitdrukkingen en Spreekwijzen.)}

\section{$\frac{\text { English. }}{\text { If you please }}$}

No, thank you

Have the goodness

Much obliged

Yes, sir (miss)

No, madam

Allow me

Bring (send) me

Excuse me

Give me

Tell me

Will you tell me?

Does anyone here speak English?

I should like to speak to you

What can I do for you?

You are too kind

Please take a seat

What do you want?

What do you mean?

I believe (think) so

I believe (think) not What do you say?

I have no objection $I k$ ben er niet tegen [to it

I beg your pardon

What did you ask?

It does not matter

Never mind

What is the matter?

Dutch.

Als het $u$ belieft

Dank $u$

Wees zoo goed

Zeer verplicht frouw)

Neen, mevrouw

Vergun mij te zeggen spreken doen?

Gij zijt te goed belieft

Wat verlangt gij?

Wat bedoelt gij?

Ik geloof (vermoed) het wel

Ik geloof van neen

Wat zegt gij?

Pardon

Wat vroegt gij?
Ja, mijnheer (juf-yah, meynhayr (yuf-

Breng (zend) $m i j \quad$ breng (zent) mey

Neem mij niet kwa- naym mey neet kwahGeef mij [lijklgayf mey [le(r)ls

Wees zoo goed mij wayss zoh goo't mey te(r)

zeg'gen

Is hier iemand, die iss heer eema(h)nt deo Engelsch spreekt? eng-e(r)lss spraykt?

Ik zou $u$ willen ik zow $\overparen{\text { eu }}$ wille(r)n

sprayken

Wat kan ik voor $u$ wa(h)t ka(h)n ik fohr ê

Ga zitten, als 't u gah zitten, a(h)lss't $\widehat{\mathrm{eu}}$ doon?

gey zeyt te(r) goo't be $(r)$ leeft

wa(h)t ferla(h)ngt gey?

wa(h)t bedoolt gey?

ik $g e(r)$ lohf (fermoot) het wel

ik $g \mathrm{e}(\mathrm{r}) \operatorname{lohf} \mathrm{fa}(\mathrm{h}) \mathrm{n}$ nayn wa(h)t zegt gey?

ik ben er neet taygen

$\mathrm{pa}(\mathrm{h}) \mathrm{rdo}(\mathrm{h}) \mathrm{n}$

wa(h)t froogt gey?

Het doet er niets toe het doot er neets too

Het komt er niet op het komt er neet op abn.

Wat is er? [aan wa(h)t iss er? 


\section{English. \\ I understand}

I do not understand

Make haste!

Come along!

Take care!

Listen!

Come in! - here!

Go away!

This way

That way

Too soon, - late

Very well

It snows

It freezes, thaws

It is very dirty

Is it slippery?

I nearly fell

Do you think it will rain?

I am afraid so

It is very windy

How cold it is !

It is very cold, warm

We must have afire

It is dark

It will soon be dark

It is a fine day

Of course

On the contrary

After all

The other day

Between ourselves

Everybody knows

That is of no consequence

I remember it

All at once
Dutch.

Pronunciation.

ik ferstah [ik be(r)greyp] het

ik ferstah het neet

hahsst $\widehat{e u} w a(h) t$ !

mahk fohrt!

$\mathrm{pa}(\mathrm{h}) \mathrm{ss}$ op !

Pas op!

Luister!

[hier! loysster!

Kom binnen! - kom binnen! - heer!

Ga weg!

Deze weg, hierheen

Die weg, daarheen

Te ganu, - laat

Heel goed

Het sneeurt

Het vriest, dooit

Het is erg vuil

Is het glad?

gah wech!

dayze(r) wech, heerhayn

dee wech, dahrhayn

te(r) gow, - laht

hayl goo't

het snay'wt

het freesst, doh'it

het iss erch foyl

iss het $g$ la(h)t?

[len

Ik ben bijna gevallen ik ben beynah ge(r)fa(h)l-

Gelooft $u$, dat het ge(r)lohft $\widehat{\mathrm{eu}} \mathrm{da}(\mathrm{h}) \mathrm{t}$ het

zal gaan regenen? $\mathrm{za}(\mathrm{h}) \mathrm{l}$ gahn rayge(r)nen?

Ik vrees van wel

Het waait erg

Wat is het koud!

Hetisergkoud, warm! het iss erch kowt, wahrm

Wijmoesten het. vuur aanleggen

Het is dontier

Het zal spoedig donker $\approx i j n$

ik frayss $f a(h) n$ wel

het wah'it erch

wa(h)t iss het kowt!

wey mooste(r)n het feur

ahnleggen

het iss donker

het $\mathrm{za}(\mathrm{h}) \mathrm{l}$ spoode $(\mathbf{r}) c h$ donker zeyn

Het is een mooie dag het iss ayn moh'i-e(r) Natuurlijk

Integendeel

Bij nader inzien

Verleden, onlangs

Onder ons gezegd

Iedereen weet.

Dat beteekent niets

Ik herinner het mij Plotseling nahtêrle(r)k $\quad[\mathrm{da}(\mathrm{h}) c h$ intaygendayl

bey nahder inzeen

ferlayden, onla (h)ngs

onder onss ge(r)zegt

eederayn wayt

$\mathrm{da}(\mathrm{h}) \mathrm{t}$ be(r)tayke(r)nt neets

ik herinner het mey

plotsse(r)ling 
Leave me alone

You are joking

You are right

Don't be angry

That depends

So much the more With all my heart

I am going to town Are you going anyUp stairs [where? Down stairs

I am in a hurry

Where have you come from?

Don't go away

I shall go home

On horseback

Neither do I

That is just like you

$\mathrm{He}$ is a friend of mine

Not long ago [you Niet lang geleden

I have come to tell Ik kom u zeggen

She is always well dressed [done

No sooner said than

I like being here

I have been told

As much as I can

I value it very much

I am used to it [me? Ik ben er aan gewoon ik ben er ahn ge(r)wohn Who is it that calls Wie roept mij? [niet wee roopt mey?

It is not my fault Het is mijne schuld het iss meyn schult neet

\section{Expressions of Surprise, Sorrow, Joy, Anger, \&c.}

(Uitdrukkingen van Verbazing, Smart, Vreugde, Toorn, \&c.)

What?

Is it possible?

Indeed !
Wat! Hoe!

Is het mogelijk!

Heusch!
Pronunciation.

laht mey met russt

gey schertst

gey hebt ge(r)leyk

wayss neet bohss $[\mathrm{a}(\mathrm{b}) \mathrm{f}$

da(h)t ha(h)ng't er fa(h)n

zohfayl te(r) mayr

fa(h)n gahnsse(r) hahrte(r)

ik gah nahr de(r) sta(h)t

gaht gey ergenss hayn?

nahr bohfen

nahr be(r)nayden

ik heb erge(r) hahst

dahn?

gah neet wech

ik za(b)l nahr hoyss gahn

te(r) pahrt

ik ayfe(r)nmin

$\mathrm{da}(\mathrm{h}) \mathrm{t}$ iss net eets fohr $\widehat{\mathrm{eu}}$ hey iss ayn freent $\mathrm{fa}(\mathrm{h}) \mathrm{n}$ neet la(h)ng ge(r)layden ik kom $\widehat{\text { eu }} z e g^{\prime} g$ en

zey iss a(h)lteyt goo't ge(r)klayt

[dahn zoh ge(r)zegt, zoh ge(r)ik ben gahrne(r) heer men hayft mey ge(r)zegt zohfayl ik ka(h)n 


\begin{tabular}{|c|c|c|}
\hline h. & ch. & a. \\
\hline e & Dat is & $\mathrm{da}(\mathrm{h}) \mathrm{t}$ \\
\hline me & $J e$ & ye(r) ferbahst \\
\hline$g !$ & & \\
\hline & & \\
\hline & & hoo ya(h) \\
\hline lad & $I k$ & ik ben hayl bley [ke \\
\hline I am ! & lulkkig! & wa(h)t ben ik ge(r) \\
\hline al! & $W a$ & wa(h)t hayrle(r)k! \\
\hline & $H i j$ & hey iss zayr bohss \\
\hline ong & $G i j$ & gey hebt groht o \\
\hline & Wel. & wel foo'i! \\
\hline d you do & Hoe kon u dat doen? & hoo kon \\
\hline or shame! [so? & Wel foei! & wel foo'i! \\
\hline ou are very much & $\begin{array}{l}\text { Gij zijt zeer te be- } \\
\text { rispen }\end{array}$ & gey zeyt z \\
\hline on' & ord niet & $a(h)$ \\
\hline
\end{tabular}

\section{Making Enquiries. (Inlichtingen vragen.)}

Do you hear me? Verstaat gij mij? |ferstaht gey mey?

Will you kindly...? Wilt gij zoo vrien- wilt gey zoh freende(r)[me? delijk zijn ...?

Do you understand Begrijpt gij mij?

What is that? Wat is dat?

Are you sure?

Zijt gij er zeker van?

Where is...? [that? Waar is ...?

What do you call Hoe heet dat?

What does that Wat beteekent dat? mean?

What is that for?

Can I see Mr. H. ? Kan ik Mijnheer $H$. spreken?

When does he re-Wanneer komt hij turn?

Please give him my Wees zoo goed hem card

I will call again to- $I k$ zaven, morgenochmorrow, between 10 and 11 a.m. tend terugkomen, tusschen tien en elf

$\mathrm{le}(\mathrm{r}) \mathrm{k}$ zeyn ? begreypt gey mey? wa(h)t iss da(h)t? zeyt gey er zayker $\mathrm{fa}(\mathrm{h}) \mathrm{n}$ ? wahr iss ...? hoo hayt da(h)t? wa(h)t be(r)tayke(r)nt $\mathrm{da}(\mathrm{h}) \mathrm{t}$ ?

wahrfohr deent da(h)t? $\mathrm{ka}(\mathrm{h}) \mathrm{n}$ ik meynhayr $\mathrm{H}$. sprayke(r)n?

wa(h)nnayr komt hey teruch?

wayss zoh goo't hem meyn kahrtye(r) te(r) gayfen

ik za(h)l, morgenochtent terugkohme(r)n, tus'sen teen en elf eur 
Notices. (Kennisgevingen.)

English.

Apartments

Bedroom for single gentleman

Entrance, exit

Fire alarm

Furnished rooms

Just published

Keep off the grass

Knock, ring

Notice! Public

Private

No admittance

No smoking allowed

No thoroughfare

Please do not touch

Please wipe your Pull, push

Refreshments

To be let

Unfurnished rooms Unfurnished bedroom to be let
Dutch.

Kamers

Slaapkamer voor een heer alleen

Ingang, uitgang Brandsein [mers Gemeubileerde kaJuist verschenen

Blijf van het gras Klop, bel

Publieks Tennis Privé [geving!

Verboden toegang

Niet rooken

Geen doorgang

Verzoeke niet aan te raken

Verzoeke voeten te

Trek, duw [vegen Terverschingen

Te huur [kamers

Ongemeubileerde

- slaapkamer te huur
Pronunciation.

kahmerss

slahpkahmer fohr ayn hayr a(h)llayn inga(h)ng, oytga(h)ng bra(h)nt'seyn [kahmerss ge(r)mu(r)beelayrde(r) yoysst ferschayne(r)n bleyf $\mathrm{fa}(\mathrm{h}) \mathrm{n}$ het $g \mathrm{ra}(\mathrm{h}) \mathrm{ss}$ klop, bel

publeeke(r) kennisspreefay [gayfing ferbohde(r)n tooga(h)ng neet rohke $(r) n$

gayn dohrga(h)ng

ferzooke(r) neet ahn te(r) rahken

ferzooke(r) foo'ten te $(\mathrm{r})$ trek, deuw

ferferssing'en

te(r) heur

[kahmerss onge $(r) m u(r)$ beelayrde(r) hêิ

\section{The Railway. (De Spoorweg.)}

(See Vocebulary 22, p. 38.)

To the station Get my luggage Take it to the cloak-room

I wish to register it

It is over weight

Here is the ticket Where is the waiting-room?
Naar het station

Haal mijne bagage

Breng het naar de garde robe

Ik wensch het aan ik wenss het ahn te(r)

te geven

Het is boven het gewicht

Hier is het biljet

Waar is de wacht- wahr iss de(r) wa(h)cht- nahr het stahseeon

hahl meyn bahgahge(r) breng het nahr de(r)

$$
\mathrm{ga}(\mathrm{h}) \mathrm{rde}(\mathrm{r}) \text { rohbe(r) }
$$

$$
\text { gayfe(r)n }
$$

het iss bohfen het ge(r)wicht

heer iss het bilyet'

kahmer? 
English.

Dutch.

Pronunciation.

Where is the book- Waar is het loket? |wahr iss het lohkett? ing-office?

- buffet?

- lavatory?

Are you going by Gaat gij met den the express? sneltrein?

Show me a time- Laat mij een spoor- laht mey ayn spohr'. table

When does the train start?

boekje zien

bookye(r) zeen

Wanneer vertrekt de wa(h)nnayr fertrekt de(r) trein?

treyn?

Can I book through Kan ik een door-ka(h)n ik ayn dohr'to ...?

gaand biljet krijgen naar...?

A first (second) class single ticket to...

How much is it?

We want a sleeping carriage

A corridor carriage

Return tickets

A carriage for ladies

A non-smoking compartment

Is this the train for ...?

Do I change anywhere on the journey?

Where must I change for ...?

Is this seat engaged ?

There is no room

Call the guard

The train is just going to star't

Open the door

- the window lilasse biljet enkele reis naar ...

Hoeveel is het?

Wijmoeten een slaapuagen hebben

Een wagen met corridor

Retourbiljetten

Een dames coupé rookien

Is dit de trein naar...? derweg overstappen? sproken?

$E r$ is geen plaats
Een eerste (tweede) ayn ayrsste(r) [twayde(r)] gahnt bilyet kreygen nahr ...? kla(h)sse(r) bilyet en$\mathrm{ke}(\mathrm{r}) \mathrm{le}(\mathrm{r})$ reyss nahr... hoofayl iss het?

wey mooten ayn slahp-

wahgen hebben

ayn wahgen met korreedohr

re(r)toorbilyetten

ayn dahmess koopay

Eer coupé niet ayn koopay neet roh-

Moet ik ergens on- moot ik ergenss onder-

Waar moet ik over- wahr moot ik ohferstappen naar...? sta(h)ppen nahr...?

Is deze plaats be-iss dayze(r) plahts be(r)sproluken?

er iss gayn plahts Roep den conducteur roop den konduktu(r) $r$

De trein is op het de( $\mathrm{r})$ treyn iss op het mint te vertiek- punt te(r) fertrekken Open de deur $\quad[$ ken ohpen de $(\mathrm{r}) \mathrm{du}(\mathrm{r}) \mathrm{r}$ - het raam - het rahm 
English,

Dutch.

Pronunciation.

Here is the station Hier is het station

What station is Welk station is

heer iss het stahseeon this?

Do we stop here? dit?

Do we alight here? Do we change carriages here?

Houden wij kier op? howden wey heer op?

Stappen wijhier uit.? sta(h)ppen wey heer oyt? Moeten wij hier moote(r)n wey heer ohoverstappen?

ferrsta(h)ppen ?

How long do we Hoe lang houden hoo la $(\mathrm{h}) \mathrm{ng}$ howde(r)n stop here?

Five minutes wij hier stil?

Vijf minuten

The goods train in De goederentrein

Send this by fast $Z$ end dit per snel- zent dit per sneltreyn train

By slow train

My luggage is lost trein

Per gewonen trein per ge(r)wohnen treyn Mijn bagage is zoek meyn bahgahge(r) iss

[naar... zook nahr... Forward it on to... Zend het dan door zent het da(h)n dobr Give me your ticket ('ieef mij uw biljet gayf mey eûw bilyet Here it is

Hier is het heer iss het

\section{The Steamboat. (De Stoomboot.)}

(See Vocabularies 21 \& 22, pages 37 to 39.)

When do we start? Hoe laat vertrekkien hoo laht fertrekken wey? When shall we Hoe laat komen wij hoo laht kohme(r)n wey Time is up! Het is tijd! het iss teyt!

Where is your lug-Waar is uwe ba-wahr iss euwe(r) bahgage?

Let us go down into the cabin

Where is my berth?

I want a cabin to myself

Look for my things There was another trunk

Come to the Custom-house

Have you your passport? gage?

Laten wij in de lahten wey in de(r) kahkajuit gaan yoyt gahn

Waar is mijn kooi? wahr iss meyn kohi? Ik wensch een hut al-ik wenss ayn hut $a(h) l^{\prime}$ leen tehebben [goed layn te (r) hebbun

Kijk eens naar mijn keyk ayns nahr meyn goo't Er was nog een er wa(h)ss noch ayn kof'koffer fer

Kom mede naar het kom mayde(r) nahr het douanenkantoor dooabne(r)nka(h)ntohr Hebt gij uw pas- hebt gey euw pa(h)ss'poort? pohrt? 
$\frac{\text { English. }}{\text { Will you examine }}$ this trunk?

Here are the keys Have you anything to declare?

I have nothing liable to duty
Wees zoo goed dezen koffer na te zien? Hier zijn de sleutels heer zeyn de(r) slu(r)telss Hebt $u$ iets aan te hebt $\widehat{\text { eu }}$ eets ahn te(r) geven?

Ik heb niets, dat ik heb neets, da(h)t abn aan rechten on- rechten onderhayfe $(\mathrm{r})$ ch derhevig is

The Custom-house De douanier officer

What is the duty? Wat zijn de rechten? Is my luggage passed?

Can I remove it ?
Is mijne bagage doorgelaten?

Kan ik ze wegnemen?
Pronanclation. iss

\section{Arrixal. (Aankomst.)}

Call a cab

Open, closed

What is the fare by the journey (by the hour), cabman?

Put my luggage in the cab

Drive me to ...

Drive quickly

Drive slower

Stop! Go on!

Wait!

I wish to get out

\section{Haal een rijtuig hahl ayn reytoych \\ Open, dicht. \\ ohpen, dicht}

Wat is de vracht wa(h)t iss de(r) fra(h)cht voor de rit (per fohr de(r) rit (per eur), uur), koetsier?

Zet. mijne bagage in zet meyn bahgahge(r) in

\section{het rijtuig}

Breng mij naar ... Rijd vlug

Rijd langzamer Houd op! Ga door! Wacht!

Ik wensch uit te stap- ik wenss oyt te(r) sta(h)ppen

\section{het reytoych} breng mey nahr... reyt fluch

reyt la(h)ng'zahmer wa d wa(h)cht! pen

\section{The Omnibus and Tram. (De Omnibus en Tram.)}

The omnibus sta- De omnibus halte de(r) omneebuss ha(h)ltion

Give me a ticket

Conductor

Inside

Outside

Geef mijeen kaartje gayf mey ayn kabrtye(r)

Conducteur

Binnen in

Boven op konduktu(r)r

bin'nen in

boh'fen op 


\begin{tabular}{|c|c|c|}
\hline glish. & Dutch. & Pronunciation. \\
\hline ' Full & "Vol" & "fol" \\
\hline & Ga & \\
\hline at. & Zet mij af in. & zet $\mathrm{m}$ \\
\hline What fare to ...? & Hoeveelkosthet tot..? & hoofayl koss \\
\hline $\begin{array}{l}\text { Is there room for } \\
\text { two inside? }\end{array}$ & $\begin{array}{l}\text { Is er binnen plaats } \\
\text { voor twee? }\end{array}$ & $\begin{array}{l}\text { iss er binnen plahts fohr } \\
\text { tway? }\end{array}$ \\
\hline $\begin{array}{l}\text { Room for three } \\
\text { outside }\end{array}$ & $\begin{array}{c}\text { Plaats voor drie } \\
\text { boven op }\end{array}$ & $\begin{array}{l}\text { plahts fohr dree bohfen } \\
\text { op }\end{array}$ \\
\hline Fares, please & $\begin{array}{l}\text { Kaartjes, als het } u \\
\text { belieft. }\end{array}$ & $\begin{array}{l}\text { kahrtye(r)s, a(h)lss het } \\
\text { eu be(r)leeft }\end{array}$ \\
\hline $\begin{array}{l}\text { Electric, horse, } \\
\text { steam tram }\end{array}$ & $\begin{array}{l}\text { Elelitrische-, paar- } \\
\text { den, stoomtram }\end{array}$ & $\begin{array}{l}\text { aylektrisse }(\mathrm{r})-, \text {, pahrden-, } \\
\operatorname{stohmtra}(\mathrm{h}) \mathrm{m}\end{array}$ \\
\hline
\end{tabular}

\section{The Hotel. (Het Hotel.)}

(See Vocabularieg $15 \& 17$, pages $28 \& 30$.)

Which is the best Wat is het beste $/ \mathrm{wa}(\mathrm{h}) \mathrm{t}$ iss het beste( $\mathrm{r}$ ) hotel ? hotel?

hoh'tel?

Let us have supper Laat ons spoedig laht onss spoode(r)ch soosoon

Are our rooms ready?

I want a bedroom soupeeren payren

Zijn onze kamers zeyn onze(r) kahmerss gereed?

Ik moet een slaap-ik moot ayn slahpkahmer kamer hebben

Let me see the Laat mij de kamer laht mey de(r) kahmer room

What is the charge?

That is too dear

\section{zien}

Wat is het tarief?

Dat is te duur

Il verlang een goed. koopere one

Have you a doublebedded room?

Are there any letters for me?

Where is the W.C.? Can I have a warm bath?

Give me the key of my room

Bring me some warm water
Hebt u een met twee bedden? Zijn er ook brieven zeyn er olk breefen fohr voor mij! [plaats?. mey?

Waar is de zekere wahr iss de(r) saykerre(r) Kan ik een warm ka(h)n ik ayn wa(h)rm bad krijgen?

Geef mij den sleutel mijner kamer?

Breng mijwat warm breng mey wa(h)t water ba(h)t kreygen?

gayf mey den slu.(r)tel meyner kahmer wa(h)ren wahter 
English.

Datch.

Pronunciation.

Take my luggage Breng mijne bagage breng meyn bahgahge(r) down

naar beneden

nahr be(r)nayden

Give me a candle, Wees zoo goed mij wayss zoh goo't mey ayn please

We leave early tomorrow

een kaars te geven kahrss te(r) gayfen

Wij vertrekken mor- wey fertrekken morgen gen vroeg

frooch

\section{Correspondence. (Briefwisseling.)}

(See Vocabulary 30, page 53.)

I must write a $\mid$ Ik moet een brief $\mid$ ik moot ayn breef schreyletter

\section{schrijuen}

Ik heb papier en ik heb pahpeer en inkt and ink

Have you any thin note paper?

Will you give me some?

Lend me a sheet

A quire

Do you like a hard or soft pen?

I like it hard (fine, broad)

Is your letter for the post?

What is the day of the month?

To-day is the first

Seal your letter

There is no wax

Take this letter to the post

Pay the postage

Newspaper wrapper

Please deliver this by hand

\section{inkt noodig nohde(r)ch}

Hebt $u$ ook dun hebt $\widehat{e u}$ ohk dun post'postpapier?

Wilt ge mij wat wilt ge(r) mey wa(h)t geven?

Leen mij een vel layn mey ayn fel

Een katerntje (pa- ayn kahterntye(r) (pahpier)

peer)

Houdt $u$ van een howt $\overparen{e u} \mathrm{fa}(\mathrm{h}) \mathrm{n}$ ayn steystijve of een slap- $\mathrm{fe}(\mathrm{r})$ off ayn $\mathrm{sla}(\mathrm{h}) \mathrm{p}$ pe pen?

pe(r) pen?

$1 k$ houd van een stij- ik howt $\mathrm{fa}(\mathrm{h}) \mathrm{n}$ ayn stey$\boldsymbol{v e}$ (fijne, breede) $\mathrm{fe}(\mathrm{r})$ [feyne(r), brayde(r)] Moet uw brief naar moot euw breef nahr de post? de(r) post?

De hoeveelste is het $\operatorname{de}(\mathrm{r})$ hoofaylste $(\mathrm{r})$ iss het vandaag? fa(h)ndahch

Het is heden de het iss hayden $\operatorname{de}(\mathrm{r})$ eerste

Verzegel uw brief ferzaygel euw breef Er is geen lak er iss gayn la(h)k

Breng dezen brief breng dayzen breef nahr naar de post

Betaal het porto de(r) post be(r)tahl het portoh koora(h)ntenstrohk Courantenstrook Wees zoo goed dit wayss zoh goo't dit zelf zelf te brengen te(r) brengen 


\section{Post-0ffice and Telegrams.}

\section{(Postkantoor en Telegrammen.)}

(See Vocabulary 31, page 53.)

English.

Dutch.

Pronunciation.

Office of origin origin

Name and address of sender

Signature

Where is the telegraph office?

Have you any letters, telegrams, for Mr....?

Will this letter go to-night?

What is the postage of this letter? To register a letter

By to-day's post

By return of post I wish to send ... Ik wilde zenden... What is the charge? Hoeveel kost het? The first ten words De eerste tien

Every additional

When does the post leave for England?

When is the next delivery?

When does the office close?

I want a Postal Order for...

Payable at the General Post Office, London

Kantoor van af- $\mathrm{ka}(\mathrm{h}) \mathrm{ntohr} \mathrm{fa}(\mathrm{h}) \mathrm{n}$ a(h)fzending

Naam en adres van nahm en ahdress $\mathrm{fa}(\mathrm{h}) \mathrm{n}$ den afzender

Handteekening

Waar is het telegraafkantoor?

Hebt gij ookbrieven, hebt gey ohk breefen, telegrammen voor Mijnheer ...?

Wordt deze brief van avond nog verzonden?

den a(h)fzender ha(h)nt-taykening wahr iss bet te(r)le(r)grahf-ka(h)ntohr? te(r)le(r)gra(h)mmen, fohr meynhayr ...? wo(h)rt dayze(r) breef fa(h)n ahfont noch fe(r) zo(h)nden ?

Hoeveel port moet is hoofayl pohrt moot ik hiervoor betalen? Een brief aanteekenen

Met de post van vandaag

Per ommegaande woorden

Ieder woord meer heerfohr be(r)tahlen? ayn breef ahntayke(r)nen

met $\operatorname{de}(\mathrm{r})$ post $\mathrm{fa}(\mathrm{h}) \mathrm{n}$ $\mathrm{fa}(\mathrm{h}) \mathrm{ndah} \mathrm{ch}$ per omme(r)gahnde(r) ik willde(r) zenden ... hoofayl kost het? de(r) ayrste (r) teen wohrden eeder wohrt mayr Wanneer vertvekt de wa(h)nnayr fertrekt $\mathrm{de}(\mathrm{r})$ post naar Enge- post nahr eng-e(r). land?

la(h)nt?

Wanneer is de vol- wa(h)nnayr iss de(r) folgende bestelling? Wanneer is het kantoor gesloten? Ik moet een postwis- ik moot ayn postwissel sel hebben voor... hebben fohr...

Betaalbaar aan het be(r)tahlbahr ahn het hoofd postkantoor hohft postka(h)ntohr te Londen te(r) londen 
$\frac{\text { English. }}{\text { Is the London mail }} \mid \frac{\text { Dutch. }}{\text { Is de mail uit iss } \operatorname{de}(\mathrm{r}) \text { mayl oyt lon. }}$ in yet? Londen al aangekomen?

Is there a foreign Kan men ook post- $\mathrm{ka}(\mathrm{h}) \mathrm{n}$ men ohk posstparcel post? paketten naar het buitenland verzen. den?

The latest delivery De laatste bestelling $\mathrm{de}(\mathrm{r})$ lahtste(r) bestel. is at... heeft om...plaats ling hayft om...plahts

\section{Breakfast. (Ontbijt.)}

(See Vocabularies $15 \& 17$, pages $28 \& 30$.

Is breakfast ready? Is het ontbigt klaar? iss het ontbeyt klahr?

Breakfast is ready Is the tea made?

Do you drink ...? This cream is sour Will you take ...? These eggs are hard

Give me the salt Pass me ....

This butter is not fresh

Bring some more -Is the coffee strong enough?

It is excellent

We want more cups

Take some more ...

A piece of toast

Cold meat

A rasher

Coffee with milk Coffee without milk or cream Remove the things Neem de tafel af genoeg? kopjes noodig sterd brood Koud vleesch

IEen sneedje spels

Koffie met melk het ontbeyt iss klahr. iss de(r) tay $g \mathrm{e}(\mathrm{r}) \mathrm{zet}$ ? drinkt $\widehat{e u}$...?

dayze(r) rohm iss zeûr

Gebruilt $u$....? ge(r)broykt $\widehat{\text { eu }}$...?

Deze eieren zijn hard dayze(r) ey-eren zeyn hart

Geef mij het zout gayf mey het zowt Geef mij ... aan gayf mey ... ahn

Deze boter is niet dayze(r) boliter iss neet versch [meer ferss

Breng nog wat breng noch wa(h)t mayr Is de koffie sterk iss de(r) kof'fee sterrk

$Z_{i j}$ is vitmuntend | zey is oytmuntent

Wij hebben meer wey heb'ben mayr kop'-

Neem nog wat ...

ye(r)ss nohde(r)ch naym noch wa(h)t

Een stukje geroo- ayn stukye(r) ge(r)rohssterrt broht kowt flayss ayn snaydye(r) spek kof'fee met melk

Koffie zonder melle kof'fee zonder melk off rohm naym de(r) tahfel $a(h) f$ 


\section{5}

Dinner. (Middagmaal.)

(Bee Vocabularies 15 \& 17, pages 28 \& 30. )

English.

Show me the billof-fare [ready?

What have you

What soup will you Gravy soup [have?

Have you any ...?

Fried soles

What wine will you have?

Here is the list

We shall dine at three o'clock

Are you hungry, thirsty?

I am very thirsty

What shall I help you to?

No, thank you

Help yourselif

Cayenne pepper

Here are spinach and broccoli

Change the plates

Give me a clean fork, knife

Bring me a glass of water

- - something to drink

A corkscrew

This wine is flat

Dutch.

Pronunciation.

Laat mij de spijs-laht mey de(r) speyss. kaart zien

Wat is er gereed? wa(h)t iss er gerayt?

Welke soep neemt $u$ ? welke(r) soop naymt $\overparen{\mathrm{eu}}$ ?

Bouillon

Hebt gij ook...?

Gebakken tong

booil'lon

hebt gey ohk ...?

$g \mathrm{e}(\mathrm{r}) \mathrm{ba}(\mathrm{h}) \mathrm{k}^{\prime}$ ken tong

Welken wijn drinkt welke(r)n weyn arinkt $u$ ?

$\widehat{\text { eu }}$ ?

Hier is de wijnkaart. heer iss de(r) weyn-kahrt

Wij eten om drie wey ayten om dree $\widehat{\text { eur }}$ uur

Hebt yij honger, hebt gey hong-er, dorst? dorst?

Ik heb ergen dorst ik heb erge(r)n dorst

Wat mag ik u wa(h)t ma(h)ch ik $\widehat{\mathrm{eu}}$ yeven?

Neen, dank $u$

Bedion uzelf

gayfen?

nayn, da(h)nk $\overparen{\text { eu }}$

bedeen êzelf

Cayenne peper |kahyenne( $\mathrm{r}$ ) payper

Hier is spinazie en heer iss speenahzee en sprutitiool [den sproytkohl

[den

Breng andere bor- breng a(h)nde(r)re(r) borGeef mij een schoone gayf mey ayn schohne(r) vork, mes fork, mess

Breng mij een glas breng mey ayn gla(h)ss water

wahter

Geef mij wat te !/ayf mey wa(h)t te(r) drintien

Een kurkentreliker ayn liurkentrekker

Deze vijn is ver-dayze(r) weyn iss ferschaald

schahlt

Tea. (Thee.)

(See Vocabularies $15 \& 17$, pages 28 \& 30.)

Tea is ready

Pour out the tea

Bring a saucer

De thee is gereed

Schenk de thee in

de(r) tay iss gerayt schenk de(r) tay in

Breng een schoteltje breng ayn schohteltye(r) 
Ring, if you please Wees zoo goed te wayss zoh goo't te(r) schellen schellen

A little more milk Nog een weinig melk noch ayn weyne(r)ch More bread Meer brood

A slice of bread and Een boterham butter

Will you take some Wilt $u$ wat koek wilt $\widehat{e u}$ wa(h)t koo'k

cake?

A small piece

Make more toast

This is excellent tea

It is late

It is not late It is still early Are you tired, sleepy?

Not at all

Not much

It is only ten

I am sleepy

It is time to go to bed

Is my room ready? Go and see Good night! (tulband) nemen ?| [tu(r)lba(h)nt] naymen? Een klein stukije Maak nog uat geroosterd brood

Dit is uitstekende thee ayn kleyn stukye(r)

mahk noch wa(h)t ge(r)rohssterrt broht

dit iss oytstaykende( $r$ ) tay

\section{mayr broht [melk} ayn bohterha(h)m

\section{Eyening. (Avond.)}

\section{Het is laat \\ het iss laht}

Het is niet laat

Het is nog vroeg

het iss neet laht

het iss noch frooch

Zijt. u moe, slape- zeyt $\widehat{\text { eu }}$ moo, slahpe(r)rig?

Volstrelt niet

Niet erg

Het is pas tien uur het iss $\mathrm{pa}(\mathrm{h}) \mathrm{ss}$ teen eur

Ik heb slaap

$\mathrm{re}(\mathrm{r}) \mathrm{ch}$ ?

folstrekt neet

neet erch

ik heb slahp

Het is tijd om naar het iss teyt om nahr bet te(r) $g a h n$

Is mijne kamer ge- iss meyn kahmer gerayt?

Ga zelf zien [reed?. gah zelf zeen

Goeden nacht! goo'de(r)n na(h)cht!

\section{The Time. (De Tijd.)}

(See Vocabulary 8, page 10.)

Please tell me the Wees zoo goed mij wayss zoh goo't mey'te(r)

\section{time}

What time is it?

Ten minutes past seven

It has just struck Het heeft juist negen nine te zeggen, hoe laat het is

Hoe laat is het?

Tien minuten over zevenen

geslagen zeg'gen, hoo laht bet iss

hoo laht iss het ?

teen meeneuten ohfer zayfe(r)nen

het hayft yoysst naygen $g e(r)$ slab!yen 
English.

Dutch.

Pronunciatior.

A quarter past one It is just upon three Half-past four

Twenty minutes to $\operatorname{six}$

Kwartier over eenen

kwa(h)rteer ohfer aynen Het is op slag van het iss op sla(h)ch fa(h)n Half vijf [drieön ha(h)lf feyf [dree'e $(r) n$ Twintig minuten twinte( $\mathrm{r}$ )ch meeneuten voor zessen

Tien minuten over teen meeneuten ohfer half zes

A quarter to eight Kwartier voor ach- kwa(h)rteer fohr a(h)chExactly 3 o'clock The clock is strik- De klok slaat At what time? [ing Op welk uur?

9.0 a.m.

7.0 p.m. Om hoe laat?

's Morgens negen/smorgenss naygen eurr uur

's Namiddags zeven snahmidda(h)chs zayuur

fen êur

\section{Health. (Gezondheid.)}

(Soo Vocabulary 18, page 25.)

Do you sleep well ?| Slaapt u goed?

I hope you will Ik hoop, dat spoesoon be better

How are you?

Pretty well

I am very well

Not very well, ill

I have a headache

- - a sore throat

- got a chill

I feel sick

Send for a doctor

I want to see a ... She has a cough

She is hoarse

Where is there a chemist's shop?

How much is the doctor's fee for a visit ?

dig beter zult zijn Hoe gaat het $u$ ? Tamelijk uel Ik ben heel wel Niet erg uel, ziek Ik heb hoofdpiin - pijn in de keel - ben verkouden Zij is schor theek? visits? slahpt $\overparen{e u} g o o^{\prime} t$ ?

ik hohp, da(h)t $\widehat{e u}$ spoode(r)ch bayter zult zeyn hoo gaht het $\widehat{\text { eu }}$ ? tahme(r)le(r)k wel ik ben hayl wel neet erch wel, zeek ik heb hooftpeyn Ik voel mij misselijli ik fool mey misse(r)le(r)k Laateendokter halen laht ayn dokter hahlen Ik wensch een ...te ik wenss ayn ... te (r) Zij hoest [zien zey hoost zey iss schor

Waar is een apo-wahr iss ayn ahpohtayk?

Hoeveel berekent de hoofayl be(r)rayke(r)nt doliter voor eene de(r) dokter fohr ayne(r) feeseete(r)? 


\begin{tabular}{|c|c|c|}
\hline English. & Dutch. & Pronunclation. \\
\hline $\begin{array}{l}\text { Have this prescrip- } \\
\text { tion made up }\end{array}$ & $\begin{array}{l}\text { Laat dit recept } \\
\text { klaarmaken }\end{array}$ & $\begin{array}{l}\text { laht dit re(r)sept klahr- } \\
\text { mahken }\end{array}$ \\
\hline $\begin{array}{l}\text { One tablespoonful } \\
\text { three times a day }\end{array}$ & $\begin{array}{l}\text { Driemaal daags een } \\
\text { eetlepel }\end{array}$ & $\underset{\text { aytlaypel }}{\text { dreemabl dahgss ayn }}$ \\
\hline $\begin{array}{l}\text { To be taken after } \\
\text { each meal }\end{array}$ & $\begin{array}{l}\text { Na elken maaltijd } \\
\text { in te nemen }\end{array}$ & $\begin{array}{l}\text { nah elken mahlteyt in } \\
\text { te(r) naymen }\end{array}$ \\
\hline $\begin{array}{l}\text { Shake the bottle } \\
\text { Poison }\end{array}$ & $\begin{array}{l}\text { Schud de flesch } \\
\text { Vergif }\end{array}$ & $\begin{array}{l}\text { schut de(r), fless } \\
\text { fergif }\end{array}$ \\
\hline $\begin{array}{l}\text { Outward applica- } \\
\text { tion only }\end{array}$ & $\left|\begin{array}{c}\text { Alleen voor uitwen- } \\
\text { dig gebruik }\end{array}\right|$ & $\begin{array}{l}\text { a(h)l'layn fohr oytwen. } \\
\text { de(r)ch ge(r)broyk }\end{array}$ \\
\hline
\end{tabular}

\section{In Town. (In de Stad.)}

(See Vocabulary 10, page 21.)

Where shall we Waar zullen wij|wahr zullen wey hayn. go?

Which is the way to...?

Where does this road lead?

To the right, left

About a mile

Go up the street heengaan?

Is it far from here? How far is it to...? Where can I get an omnibus? Go quickly - slowly

Let us go

Show me the way Turn to the right Keep to the left Second turning Take the first to the left and the second to the right Cross the road Is this the way to...? In what street are In uelke straat zijn gahn?

Wat is de reg

naar...?

wa(h) $t$ iss $\operatorname{de}(\mathrm{r})$ wech nahr...?

Waar gaat deze wey wahr gaht dayze(r) wech heen?

Rechts, links

Ongeveer een mijl

Ga de straat op

hayn?

rechts, links

on-ge(r)fayr ayn meyl

gah de(r) straht op

Is het ver van hier? iss het fer $\mathrm{fa}(\mathrm{b}) \mathrm{n}$ heer?

Hoeverishetnaar..? hoo fer iss het nahr ...?

Waar kan ik een wahr ka(h)n ik ayn om-

omnibus krijgen? neebus kreygen?

Loop vlug

- langzaam

Laat ons gaan

Wijs mij den weg

Sla rechts om

Houd links aan

Tweede straat

lohp fluch

- la(h)ngzahm

laht onss gahn

weyss mey de(r)n wech

slah rechts om

howt links ahn

twayde(r) straht

Sla de eerste straat slah de(r) ayrste(r) straht links en de twoede rechts in

Steek den weg over stayk de(r)n wech ohfer Is dit de weg naar...? iss dit de(r) wech nahr...? the best shops? de beste winkels? links en de(r) twayde(r) rechts in

in welke(r) straht zeyn de(r) heste $(r)$ winkelss? 
English.

Dutch.

Pronunciation.

Please direct me to ...

Which [tram] must I take for...?

Do you know Mr. ..?

I don't know anyone of that name

$\mathrm{He}$ is a friend of $H i j$ is een vriend mine [well van mij

I know him very Ik ken hem zeer goed ik ken hem zayr goo't

Where does helive? Waar woont hij?

Close by

Dichtbij

den weg te wijzen

naar...

Can you direct me Kunt u mij een goed kunt ê mey ayn goo't to a good hotel? hôtel aanwijzen? Is Mr. ..., Mrs. Is Mijnheer..., Me- iss meynhayr ..., me(r)..., at home?

Good morning How do you do?

I must go Good-bye, farewell Good evening Good night

vrouw ..., tehuis?

Goeden morgen

Hoe vaart u?

Ik moet gaan

Adibu, vaarvel

Goeden avond

Goeden nacht wahr wohnt hey?

dichtbey

de(r)n wech te(r) wey. zen nahr...

[tra $(\mathrm{h}) \mathrm{m}$ ] moot ik naymen nahr ...?

kent $\widehat{e u}$ me(r)nhayr ...? deen nahm

hey iss ayn freent $\mathrm{fa}(\mathrm{h}) \mathrm{n}$ mey frow ..., te (r)hoyss? goo'den mohrgen hoo fahrt $\overparen{e u}$ ? ik moot gahn ahdee'u(r), fahrwel gooden ahfont goode(r)n na(h)cht

\section{Shopping. (In een Winkel.)}

(See Vocabularies 6, 7, 14 to $17, \&$ 20.)

How much is it? That is too much What is the price? Will you send them at once?

I wish to buy...

I will take this Send them to... I want some calico

I should like to see Laat mij lint some ribbons
Hoeveel kost dat?

Dat is te veel

Wat kost dat?

Wilt $u$ dis dadelijk zenden?

Ik wensch ... te koopen

Ik neem dit

Zend ze naar...

Ik heb kitoen

noodig hoofayl kost da(h)t?

$\mathrm{da}(\mathrm{h}) \mathrm{t}$ iss te(r) fayl

wa(h)t kost da(h)t?

wilt eu dee dahde(r)le(r) $k$ zenden?

ik wenss ... te(r) koh. pen

ik naym dit

zent ze(r) nahr ...

ik heb kahtoon noh. $\operatorname{de}(\mathrm{r}) c h$

laht mey lint zeen 
Dutch. Pronunciation.

This colour is too dark, light

Have you any narrower, broader?

What is this a It is faded [yard? Show me some cotton

Some gloves

- others

Not so fine

This will do

Sewing-silk

A skein

Mixed pins

Tape; a bodkin

What are they a pair?

It is too dear

Try on these

They fit you very well [with me

I will take them Send this home directly

The bill
Deze kleur is te dayze(r) $\mathrm{klu}(\mathbf{r}) \mathrm{r}$ iss te(r) donker, te licht. donker, te(r) licht Hebt $u$ ook smaller, hebt $\widehat{e u}$ ohk sma(h)ller, breeder?

Wat kost dit de el?

Het is verschoten

Laat mij katoen zien

Handschoenen

Andere

Niet zulke mooie

Deze zijn goed

Naaizijde

Een streng

Spelden ran rer-

schillende grootte schille(r)nde(r) grohte(r) band; een rijgpen $\mathrm{t}$.| ba(h)nt ; ayn reygpe(r)n Hoeveel kosten ze hoofayl koste(r)n ze(r) het paar?

Dat is te duur

Pas deze eens aan

Zij passen $u$ zeer goed

Ik zal ze meenemen Zend dit dadelijk naar huis

De rekening brayder?

wa(h)t kost dit de(r) el? het iss ferschohten

laht mey kahtoon zeen

ha(h)ndschoonen abnde(r)re(r)

neet zulke(r) moh-i-e(r) dayze(r) zeyn goo't

nah'i-zeyde(r)

ayn streng

spelde(r)n $\mathrm{fa}(\mathrm{h}) \mathrm{n}$ ferhet pahr?

$\mathrm{da}(\mathrm{h}) \mathrm{t}$ iss te(r) deur

pa(h)ss dayze(r) ayns ahn

zey pa(h)ss'en $\overparen{\text { eu }}$ zayr goo't

[men

ik $\mathrm{za}(\mathrm{h}) \mathrm{l}$ ze(r) maynayzent dit dahde(r)le(r)k nahr hoyss de(r) rayke(r)ning

\section{The Dressmaker. (De Naaister.)}

(See Vocabulary 14, p. 27.)

Here is the dress- Hier is de naaister / heer iss de(r) nah'ismaker

Show her in [komen] te(r)

Have you brought Hebt u mijne japon hebt $\widehat{\text { eu }}$ meyn yahpon my dress?

Here it is

Will you try it on? It fits you well

The sleeves are not wide enough meegebracht?

Hier is ze

[sen? heer iss ze(r) [pa(h)ssen?

Wilt $u$ deze aanpas- wilt $\widehat{e u}$ dayze(r) ahn$Z i j$ past u goed zey pa(h)st eu goo't

De mouwen zijn niet $\mathrm{de}(\mathrm{r})$ mowen zeyn neet wijd genoeg weyt $g \mathrm{e}(\mathrm{r})$ nooch 
English.

The skirt is too De rok is te nauw narrow

Add another breadth

It is short-waisted

It is long-waisted Make all these alterations

What trimming would you put on? Light blue ribbon You shall have it $U$ zult het Zaturdag on Saturday

Without fail [me Zonder mankeeren Don't disappoint Stel mij niet teleur stel mey neet te(r)lu(r)r

Doe er nog eene doo $\mathrm{e}(\mathrm{r})$ noch ayne(r) baan bij bahn bey

$H i j$ is te kort van hey iss te(r) kort fa(h)n taille

- - langvan taille _ - la $(\mathrm{h}) \mathrm{ng} \mathrm{fa}(\mathrm{h}) \mathrm{n}$

Maak al deze ver-|mahk a(h)l dayze(r) feranderingen

Welk garnituur zoudt $u$ aanraden?

Lichtblauw lint $\mathrm{a}(\mathrm{h})$ ndering-en

welk gahrneeteur zowdt eu' ahnrahden?

lichtblow lint

$\widehat{\mathrm{eu}}$ zult het zahtur$\mathrm{da}(\mathrm{h})$ ch hebben zonder ma(h)nkayren

\section{The Shoemaker. (De Schoenmaker.)}

(For Shopping see page 109.)

I wish to see some $I k$ wensch eenigelik wenss ayne(r)ge(r) shoes

Ladies', gentle- Dames-, heeren-, men's, children's kinderschoenen enz

The soles are rather De zolen zijn tamethick, thin

They are too tight, short, long

Heels too high

I cannot get my foot in

lijk dik, dun

Zij zijn te nauw, kort, lang

Te hooge halkken

Ik kan er mijn voet. niet in krijgen

Here is a shoe-horn Hier is een hoorn

They hurt my heel, toes, instep

Zij doen mijn hiel, teenen, wreef, zeer schoonen te(r) zeen dahmess-, hayren-, kinder-schoonen enz

de(r) zohlen zeyn tahme(r)le(r)k dik, dun zey zeyn te(r) now, kort, la(h)ng te(r) hohge(r) ha(h)kken ik ka(h)n er meyn foo't neet in kreygen heer iss ayn hohrn zey doon meyn heel, taynen, w-rayf, zayr.

I cannot walk in $I k$ lian er niet in ik ka(h)n er neet in them.

Take my measure Neem mij de maat naym mey de(r) maht Imusthave themas $I l$ moet $z e$ zoo ik moot ze(r) zoh spoosoon as possible spoedig mogelijk de(r)ch mo(h)ge(r)le(r)k hebben $\quad$ hebben 
The Laundress. (De Waschurouro.)

(See Vocabulary 14, p. 27, and Washing List, below.)

$\frac{\text { English. }}{\text { This is too limp }}$

You don't put enough starch

I miss a collar

See how badly that is ironed, starched

You have scorched, torn, this dress

You put too much blue in my linen

This is not $\mathrm{my}$ handkerchief

Have you your bill? I will pay you

I want this linen washed

When can I have it ?

Please return this linen on ...

You must bring back this list

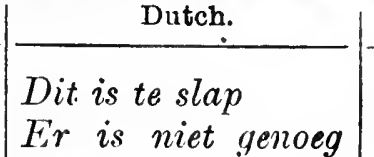

stijfsel in [(boord)

Ik mis een lraag ik miss ayn krahch Tie eens, hoe slecht zee as 'Zie eens, hoe slecht zee aynss, hoo slecht dit. dit is gestreken, iss ge(r)strayken, ge(r)gesteven

$U$ hebt deze japon geschroeid, gescheurd

$U$ doet te veel blauw- $\overparen{\text { eu }}$ doot te(r) fayl blousel sel in mijn linnengoed

Dit is mijn zalidoeli dit iss meyn za(h)kdook niet [ning? neet

Hebt $u$ uwe reke- hebt $\overparen{\text { eu }} \overparen{\text { eutwe(r) ray- }}$ Ik zal u betalen

ik za(h)l ề be(r)tahlen

It wensch dit lin-ik wenss dit linne(r)n nen gewasschen te hebben ge(r)wa(h)ssen te(r). hebben

Pronunciation.

dit iss te(r) sla(h)p

eu liebt dayze(r) yahpon ge(r)schrooit, ge(r)schurt in meyn linne(r)ngoo't

Wanneer kan ik het wa(h)nnayr lia(h)n ik het livijgen? kreygen?

\section{Washing List.* (Waschlijst.)}

FOR GENTLEMEN. (Voor Heeren.)

Collars

Cuffis

Flannel waistcoat

Neckties

Night shirts

Pants (drawers)
Kragen, boorden m. krahgen, bohrden

Manchetten f.pl.

Flanellen vest $\mathrm{n}$.

Dassen f.pl.

Nachthemden n.pl. na(h)cht-hemden

Onderbroeken f.pl. onderbroo'ken

* THIMM (C. A.) Dutch Washing Book for Ladies and Gentlemen. With Counterfoils in English, alphabetically arranged for the use of Tourists and others. Red wrapper, 8vo. Bd. 


\section{3}

\begin{tabular}{|c|c|c|}
\hline h. & Dutch. & Pronunciation. \\
\hline $\begin{array}{l}\text { Pocket handker- } \\
\text { Shirts } \quad \text { [chiefs } \\
\text { Silk handkerchiefs } \\
\text { Socks } \\
\text { Stockings } \\
\text { Under-vest } \\
\text { Waistcoat (vest) }\end{array}$ & $\begin{array}{l}\text { Zakdoeken m. } \\
\text { Hemden } \\
\text { inden zakdoeken } \\
\text { Sokken f. } \\
\text { Kousen f. } \\
\text { Flanel n. } \\
\text { Vest }\end{array}$ & $\begin{array}{l}\text { za(h)kdooken } \\
\text { hemden } \\
\text { zeyden za(h)kdooken } \\
\text { sokken } \\
\text { kowssen } \\
\text { fla(b)nell } \\
\text { fest }\end{array}$ \\
\hline & FOR LADIES. (Voor & Dames.) \\
\hline $\begin{array}{l}\text { Apron } \\
\text { Bodice } \\
\text { Cap } \\
\text { Chemise } \\
\text { Drawers } \\
\text { Dress } \\
\text { Dressing-gown } \\
\text { Night cap } \\
\text { Night gown } \\
\text { Petticoats } \\
\text { - flannel } \\
\text { Silk stockings } \\
\text { Sleeves } \\
\text { Stays (corsets) } \\
\text { Napkins } \\
\text { Towels }\end{array}$ & \begin{tabular}{|l|} 
Schortje, boezelaar \\
Japonlijf $\mathrm{n}$. \\
Muts f. \\
Hemd $\mathrm{n}$. \\
Onderbroek f.pl. \\
Japon f. \\
Peignoir \\
Slaapmuts f. \\
Nachtjapon \\
Rokken m. \\
Flanellen rokken \\
Zijden kousen \\
Mouwen \\
Corset \\
Servetten \\
Handdoeken m.pl.
\end{tabular} & $\begin{array}{l}\text { schort-ye(r), booze(r)lahr } \\
\text { yahpo(h)nleyf } \\
\text { muts } \\
\text { hemt } \\
\text { onderbroo'k } \\
\text { yahpon } \\
\text { paynwahr } \\
\text { slahpmuts } \\
\text { na(h)cht-yahpon } \\
\text { rokken } \\
\text { fla(h)nellen rokken } \\
\text { zeyden kowssen } \\
\text { mowen } \\
\text { korset } \\
\text { serfetten } \\
\text { ha(h)ndoo'ken }\end{array}$ \\
\hline
\end{tabular}

Cycling Phrases. (Uitdrukkingen voor Wielrijders.) See Vocabulary 25, page 45.)

I want my machine Ik heb mijn wicl ik heb meyn weel nohnoodig $\mathrm{de}(\mathrm{r}) \mathrm{ch}$

Fitted with all Voorzien ran alle fohrzeen $\mathrm{fa}(\mathrm{h}) \mathrm{n} \mathrm{a}(\mathrm{h}) \mathrm{lle}(\mathrm{r})$ accessories toebehooren toobe(r)hohren

I wish to hire ... Il wensch...te huren ik wenss ... te $(\mathrm{r})$ heurren By the hour, day Bij het uur, den dag bey het eùr, den da(h)ch What are your Wat is uw prijs? $\mathrm{wa}(\mathrm{h}) \mathrm{t}$ iss euw preyss? terms?

Have you a cycle Hebt $u$ een berg- hebt $\widehat{e u}$ ayn bergplahts shelter? plaats voor wielen? fohr weelen?

To tighten-loosen Een moer aanschroe- ayn moo'r ahnschroofen -a nut ven-losschroeven -los'schroofen 
English.

Dutch.

Pronunciation.

Are the roads good or bad?

The chain is off

Zijn de wegen goed of slecht?

De ketting is los

I must inflate my Ik moet mijne bantyres

I have broken ...

Are you ready?

We will start at ...

Ring your bell

My tyre is punc- Mijn band is lek

Make haste! [tured Haast $u$ !

Stop! Look out!

Don't go so fast

Keep straight on

We must light up

My lamp requires

a new wick

[guide?

Have you a road Yes; which one do you require?

The one for Amsterdam and environs

I want my machine cleaned

Where can I get my bicycle mended?

Can you repair this for me?

How long will it take?

I am a member of the London Cyclists' Touring Club; here is my ticket

den oppompen

Ik heb ...gebroken

Zijt gij gereed?

Wij zullen om ...

vertrekken

Bel eens

Stop! Kijk uit!

Loop niet zoo vlug

Ga recht uit

iVij moeten onze lan-

taarns aansteken hebben? en omstreken te laten schoonmaken reeren? duren? Londensche Wielrijders Club; hier. is mijn bewijs van lidmaatschap
Ik heb een nieuwe ik heb ayn neewe(r) pit pit voor mijnlamp fohr meyn $\mathrm{la}(\mathrm{h}) \mathrm{mp}$ noodig [kaart? nohde(r)ch [kahrt?

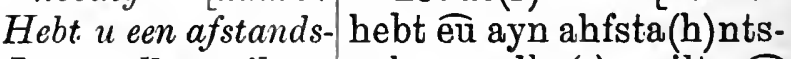
$\mathrm{Ja}$; welke wilt. "yah; welke(r) wilt $\overparen{\mathrm{eu}}$ hebben?

Die voor Amsterdam dee fohr a(h)msterda(h)m

en omstrayken

Ik wensch mijn fiets ik wenss meyn feets te(r)

Waar kan ik mijn wahr ka(h)n ik meyn fiets laten repa- feets lahten $\mathrm{re}(\mathrm{r}) \mathrm{pah}$ rayren?

Kunt $u$ dit voor mij liunt $\widehat{\mathrm{eu}}$ dit fohr mey - repareeren? re(r)pahrayren?

Hoe lang zal het hoo la(h)ng $\mathrm{za}(\mathrm{h}) \mathrm{l}$ het

deure(r)n?

Ik ben lid van de ik ben lit fa(h)n de(r) londensse $(r)$ weelreyderss klub; heer iss meyn be(r)weyss $\mathrm{fa}(\mathrm{h}) \mathrm{n}$ litmahtscha(h)p 
Motoring Phrases. (Gesprekken over Automobielen.)

(For Vocabulary see page 49, also Cycling, pages 45 \& 113.)

English

Have you examined my speed-changing gear?

Replace my brake The steering-gear is out of order

I want a carburator for voiturette

My back spring is broken

Change it

A pinion is wanted for the small axle

I have lost my

I have lost my horn (hooter)

Have you an accumulator-four volts?

You must fix the radiator to the frame

Give me a petrol tube

An automobile map on cloth

What is the power of this motor?

This is an eighthorse-power engine

Is it a good hillclimber?

I want an experienced driver ken? orde gebrolien loren volts? leumbuis
Dutch.

Hebt u mijne ver-hebt $\widehat{e u}$ meyn fe(r)rsnelling nageke-

Verzet mijn rem

Het sturur is niet in het steur iss neet in

Ik heb een carbura-ik heb ayn karbeurahtor tor noodig voor een klein rijtuig

Mijn achterveer is

Neem een andere

$\mathrm{Er}$ is een rondsel er iss ayn rondsel rehnoodig voor de kleine as

Ik heb mijn hoom ik heb meyn hohrn (blaashoorn) ver-

(blahsshohrn) ferlohren

Hebt $u$ oen accu-hebt $\widehat{\text { eu }}$ ayn $a(\mathrm{~h}) \mathrm{k}$ keumulator - vier

$U$ moet het radieer- $\widehat{e u}$ moot het raydeeayrtoestel aan het toostel ahn het rahm raam bevestigen be(r)feste(r)gen

Geef mij een petro-

Een áutomobiel kaart op linnen Welke kracht. heeft welke(r) $\mathrm{kra}(\mathrm{h})$ cht hayft deze motor?

Dit is een acht paardekracht machine

Kan men er goed mee steigen?

Ik heb een erva-ik hep ayn erfahre(r)r ren bestuurder (chauffeur) noodig meulahtor - feer foltss?

gagf mey ayn paytrohle(r)umboyss

ayn ohtohmoh'beel kahrt op linnen

dayze(r) mohtor?

dit iss ayn a(h)cht pahrde(r)kra(h)clit mahsheene $(r)$

(h)n men er goo't máv steyge(r)n? besteurde(r)r [shohf$\mathrm{fu}(\mathrm{r}) \mathrm{r}]$ nohde(r)ch 
Photographic Phrases. (Uitdrukkingen aan het Fotografeeren.) (For Vocabulary see p. 49.)

English. Dutch.

Pronunciation.

Must permission be obtained to photograph?

Moet verlof worden moot ferlof worden ahnaangevraagd om te $g \mathrm{e}(\mathrm{r})$ frahgt $\mathrm{om}$ te $(\mathrm{r})$ fotografeeren?

I wish to take the Ik wensch het inwen- ik wenss het inwende(r)interior

dige van het huis te nemen

What exposure Hoe lang moet ik hoo la(h)ng moot ik de(r) must I give it? de opname laten opnahme $(\mathrm{r})$ lahte $(\mathrm{r}) \mathrm{n}$ duren?

The light is too Het licht is te sterk het licht iss te(r) sterk strong

It is out of focus

What size of plate do you use?

I only use quarterplate

[brandpunt

Het is niet in het het iss neet in het Welke grootte van welke(r) grohtte(r) $\mathrm{fa}(\mathrm{h}) \mathrm{n}$ plaat gebruikt $u$ ? plaht ge(r)broykt $\overleftrightarrow{\mathrm{eu}}$ ?

Ik gebruik alleen een ik ge(r)broyk a(h)llayn kwartplaat

Have you a dark Hebt gijeen donkere hebt gey ayn donkere(r) room?

I want to develop some plates

What a beautiful photograph!

It is a perfect picture kamer? kahmer?

Ik wensch eenige pla- ik wenss ayne(r)ge(r) plahten te ontwikkelen ten te(r) ontwikke(r)len Wat eene prachtige wa(h)t ayne(r) pra(h)chfotografie! te(r)ge(r) fohtograhfee! Het is eene rol-het iss ayne(r) folmahkmaakte afbeelding te(r) a(h)fbaylding

This plate is spoilt Deze plaat is be- dayze(r) plaht iss be(r)dorien dorfen

That is due to over-Dat is aan te lange $\mathrm{da}(\mathrm{h}) \mathrm{t}$ iss ahn te(r) exposure

I am going to print more copies

This was spoilt in the printing

opname te wijten

la(h)ng-e(r) opnahme(r) te(r) weyten

The grouping is De groepeering is very good

I have secured some beautiful views zeer goed

Ik heb eenige fraaie ik heb ayne(r)ge(r) frah'igezichten verkregen $\mathrm{e}(\mathrm{r})$ ge(r)zichten ferkraygen 
Commercial and Trading. (Handelstermen.)

(For Vocabulary see page 41.)

English.

Dutch.

Pronunciatiou

Where can I ob- Waar kan ik een wahr ka(h)n ik ayn tain a commer- handelsreizigers ha(h)nde(r)lsreyze(r). cial traveller's vergunning krij- gerss fergunning kreypermit?

What is the fee for Wat kost eene ver- wa(h)t kost ayne(r) ferthe permit?

I represent the firm of...

gunning? gunning?

May I show you Mog illuni... mon- ma(b)ch ik meyn my samples? sters laten zien? monstersslahte(r)n zeen Send our account Zend onze rekening zent onze(r) raykening in monthly

Give me a receipt

Messrs. ... have failed

maandelijks in

Geef mij een ont- gayf mey ayn ontfa(h)ngvangbewijs

De Heeren ... ziju de(r) hayren ...... zeyn failliet.

What assets [liabi- Hoe groot is het hoo groht iss het $\mathrm{a}(\mathrm{h}) \mathrm{k}$ lities] are there?

Show me a balancesheet

There is an error in your account

$I$ shall send in a claim

Will you accept a bill?

at three months

What dividend is declared?

Your luggage is liable to duty

This cheque needs endorsing

Have you declared the value?

I will prepay the carriage

Quote me a price aktief [passief ]? teef $[\mathrm{pa}(\mathrm{h})$ sseef $]$ ?

Toon mijeen balans tohn mey ayn bah-

$$
\text { la(h)nss }
$$

Er is een abuis in er iss ayn ahboyss in êuwe rekening

we $(r)$ raykening

Ik zal eene vorde- ik $\mathrm{za}(\mathrm{h}) \mathrm{l}$ ayne(r) forde(r)ring inzenden

ring inzenden

Wilt $u$ een wissel wilt $\overparen{\mathrm{eu}}$ ayn wisse( $\mathrm{r}) \mathrm{l}$ accepteeren?

$\mathrm{a}(\mathrm{h})$ kseptayren?

- op drie maanden

Welk dividend is welk deefeedent iss fer. verklaard?

Uwe bagage is aan euwe( $\mathrm{r}$ ) bahgahge( $\mathrm{r})$ iss rechten onderhevig ahn rechten onderhayfe $(\mathrm{r}) \mathrm{ch}$ Deze cheque moet dayze(r) shek moot worworden geëndosseerd den ge(r)endossayrt Hebt $u$ de waarde hebt eu de(r) wahrde(r) gedeclareerd? $g e(r)$ dayklahrayrt? Ik zal de vracht ik $\mathrm{za}(\mathrm{h}) \mathrm{l}$ de(r) fra(h)cht vooruitbetalen fohroyt-be(r)tahlen Noteer ons een prijs nohtayr onss ayn preyss 
Dutch.

Pronanciation.

Is there any duty Is er eenig recht op iss er ayne(r)ch recht op on samples? monsters?

monsterss?

Please make me Wees zoo goed mij wayss zoh goo't mey out a list of the samples I carry

Indicating quality Aantoonende kwali-ahntohne(r)nde(r) kwah-

and quantity teit en lisuantiteit leeteyt en kwa(h)nteeteyt ayne(r) leyst op te(r) eene lijst op t.e maken van de monsters, die iti medeneem mahken $\mathrm{fa}(\mathrm{h}) \mathrm{n}$ de(r) monsterss, dee ik mayde(r)naym

\section{Changing Money. (Geld wisselen.)}

Where can I get Waar kan ik geld wahr ka(h)n ik gelt wis. money changed?

Where is the bank (a moneychanger)?

wisselen?

Waar is de bank wahr iss de(r) ba(h)nk se(r)len? (een geldwisselaar)?

I want change for $I k$ wou een bank-ik wow ayn ba(h)nkbila five - pound note

Will you give me Dutch money?

What is the exchange on English money?

Will you change me these sovereigns?

Will you cash this cheque for me?

Please give me gold and silver, not paper money

A bill of exchange

It is payable at sight

Give me the amount in ...

Have you an agent Hebt $u$ cen agent

biljet van vijf pond wisselen

Wilt $u$ mij Hol- wilt $\widehat{\text { eu }}$ mey holla(h)ndss landsch geld geven?

Wat is de koers van wa(h)t iss de(r) koorss Engelsch geld?

Wilt $u$ deze souve- wilt $\widehat{e u}$ dayze(r) soofe( $(\mathrm{r})$ reinen (ponden) voor mij wisselen?

Wilt $u$ deze cheque voor mij inwisselen?

Wees zoo goed mij goud en zilver te geven, geen papieEen wissel [ren geld $H i j$ is betaalbaar op zicht

Geeft mijhet bedrag gayf mey het be(r)in ... $\quad \operatorname{dra}(\mathrm{h})$ ch in ... reynen (ponden) fohr mey wisse(r)len?

wilt $\overparen{\text { eu }}$ dayze(r) shek fohr mey inwisse(r)len?

wayss zoh goo't mey gowt en zilfer te(r) gayfen, gayn pahpeehey iss be(r)tahlbahr op zicht [in ...? ayn wisse(r)] [ren gelt 
MONEY. (Geld.)

\section{Dutch Money, with English and American Equivalents.}

[NoTE.-These equivalents are subject to the fluctuations in exchange.]

DUTCR.

10 gulden (or guilders)

\section{GOLD.}

ENGLISH.

$\begin{array}{lll}\text { s. } & \text { s. } & \text { d. } \\ 0 & 16 & 8\end{array}$

AMERICAN. dols. cents.

\section{SILVER.}

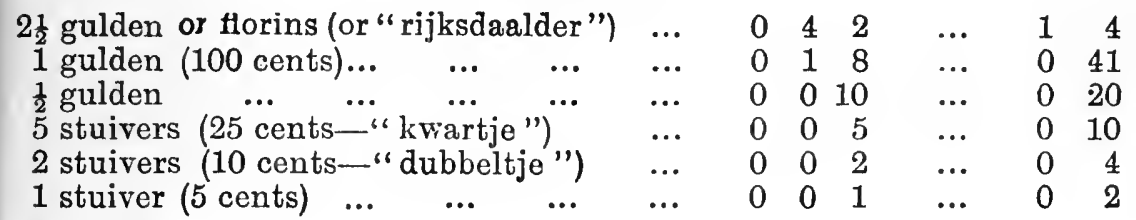

\section{BRONZE.}

$2 \frac{1}{2}$ cents...

100 cents $=20$ stuivers $=1$ gulden (or florin).

PAPER.

Notes are issued by the Bank of Netherlands for 10, 25, 40, $50,60,100,200,300,500$, and 1,000 gulden.

\section{English and American Money, with Dutch Equivalents.}

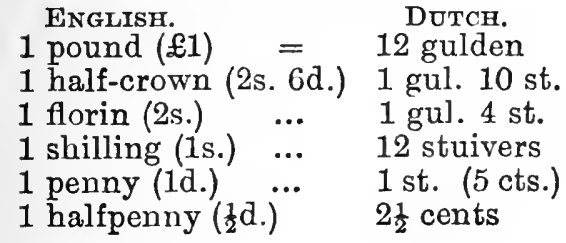

AMERICAN.

100 dollars $\quad=241$ gul. 5 st. $10 \quad, \quad \ldots \quad \ldots \quad 24$ gul. $2 \frac{1}{2}$ st.

$1 \quad, \ldots \quad \ldots \quad 1$ gul. 8 st.

50 cents ... $\quad . . \quad 1$ gul. 4 st.

$10,, \quad \ldots \quad \ldots \quad 5$ stuivers

1 cent $\ldots . . \quad \ldots \quad 2 \frac{1}{2}$ cents

Exchange.-The rate of exchange ordinarily varies from about 12 gulden $2 \frac{1}{2}$ cents to 12 g. $12 \frac{1}{2}$ c. to the English pound. 


\title{
WEIGHTS AND MEASURES.
}

\author{
(Maten en Gewichten.)
}

The Metric system is used throughout Holland for all weights and measures ; the old nomenclature is sometimes used in small towns and villages.

\section{MEASURES OF LENGTH. (Lengtemaat.)}

English. Dutch

1 inch $=2.5399$ duimen (centimeter)

1 foot $=30$ duimen

1 yard $=91$ duimen

1 furlg. $(220$ yds.) $=201$ ellen ('meter)

$1 \mathrm{mile}(1760 \mathrm{yds}$ ) $=1 \mathrm{mijl}$ (kilometer) 610 meter

5 miles $=8$ mijlen (kilometer)
Dutch.

1 duim (centimeter) $=0.394$ in. 30 duimen,$\quad=1$ foot

$1 \mathrm{el}$ (meter) $=3 \mathrm{ft} .3 \mathrm{in} . ; 1.093$ yds.; $39.37 \mathrm{in.}$

1 honderd meter (hectometer)

$=109$ yds. $1 \mathrm{ft}$.

1 mijl (kilometer) $=1093 \mathrm{yds} .2 \mathrm{ft}$. 5 mijlen,$\quad=3$ miles $220 \mathrm{yds}$.

\section{WEIGHTS. (Gewichten,)}

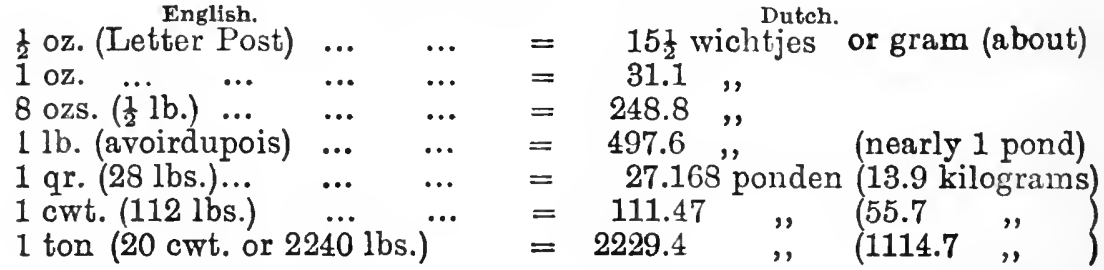

Dutch.
English.

1 wichtje $(\operatorname{gram}) \quad \ldots \quad \ldots \quad \ldots \quad \ldots \quad \ldots=15.432$ grains

100 wichtjes $\ldots, \quad \ldots \quad \ldots \quad \ldots \quad \ldots \quad \ldots \quad \ldots=3.2$ ozs.

$250 \quad, \quad \quad \ldots \quad \ldots \quad \ldots \quad \ldots \quad \ldots \quad \ldots=8 \quad \ldots \quad \ldots \quad\left(\frac{1}{2} l b.\right)$

1 pond (500 wichtjes or $\frac{1}{2}$ kilo. $\left.{ }^{*}\right) \ldots \quad \ldots \quad \ldots=1 \mathrm{lb}$. " (about)

100 ponden $\left(50\right.$ kilo.) ... $\quad \ldots \quad \ldots \quad \ldots \quad \ldots \quad \ldots=100 \mathrm{lbs} .7 \frac{1}{2}$ ozs.

$1000 \quad, \quad(500,,) \ldots \quad \ldots \quad \ldots \quad \ldots \quad \ldots=1004$ lbs. 11 ozs.

(8 cwt. 3 grs. 24 lbs.)

L.IQUIDS, \&C. (Vlocistoffen, enz.)

English. Dutch.

1 pint $=0.57 \mathrm{kan}$ (liter)

1 quart $(2$ pts.) $=1.14$ kan (liter)

1 gallon $(4$ qts. $)=4.5434 \mathrm{kan}$,

1 peck $(2$ gals. $)=9 \mathrm{kan}$ ",

1 bushel $(8$ gals. $)=36 \mathrm{kan}$.,
Dutch.

1 English.

1 (liter) $=1.76$ pints

5 kan ," = 1 gallon 1 pint

$50 \mathrm{kan}, "=11$ gallons

$100 \mathrm{kan} \mathrm{,,} \mathrm{=} 22$ gallons

( $2 \frac{3}{4}$ bushels)

"Tho word "kdlogram" is always abbreviated to "kdlo." 


\section{DANISH SELF-TAUGHT. (Thimm's System.)}

Crn. 8yo. Blue wrapper, 3:-

13 W W. F. HARVEY, M.A. With English Phonetic Pronunciation. Containing Vocabularies, Cohversational Phrases and Sentences, Elementiry Grammar, Commercial, Trading and Legal Terms, Travel Talk, Shooting and Fishing, Photography, Cycling, \&c.; Money, with jllustration of Danish Coinage, and Euglish and American values; Weights and Measures.

\section{Marborough's TRAVELLERS' PRACTICAL MANUALS CP}

\section{CONVERSATION.}

(BUB.TCCTS ARRANGED ALPHABETICALTT.)

Containing Trapel Notes, Classified Conversations-liailway, Stermer, Hotel, Tuggago. Cuntoms, Sho pung, Sight-seeing, Motoring, Cycling \&c. Mruney, Weights and Measures. Compreliensive Dictiomary of Words in Dails Use.

No. 1. ENGLISH, FRENCH, GERMAN and ITALIAN. (In one bonk.)

No. 2. ENGLISH, FRENGH, GERMAN and DUTCH. (In one lool.)

Crowis $12 \mathrm{mo}$, wrapjer, $1 / 9$ net each; cloth, 3/-net each.

\section{TRAVELLERS' ESPERANTO MANUAL OF CONVERSATION.}

\section{English-Esperanto.}

By J.C. OCONNOJ, m.A., PH.D. Containing Pronuneintion, The Accent, The Articln, Classified Conversations (subjects arranged alphabeticaily) - larilway, Steaner, llote! \&.c.. Forms of Letters, Tables of MIoney, with the Wnglish equivalents and Esperantn equivalents, Weights and Measures, 'The Numerals, Comaprehensivi Vocabulary of words in daily use.

Crown 12 mo. Green cloth. 1s. net. Wrapper, 6d. net

\section{For the STUDY OF FIRECIII.}

PRICES NET.

FRENCR SELF-TAUGH'. (Thimm'system.) Crn 8yo. Blue wrapper. it. d cloth 3 .

Bs the natural method, with Phonctic Pronunciation. Enlarged and Kevised by J. LA FFITTE, I3.-ès-L. (Principal French Master City of London School). Containin Alphabet and Pronunciation, The Nasal Sound, Notes on Articles, Gender, Accent. \&c., Classitied Vocalularies, Conversational Phrases and Sentences, 'Travel Talk, Commercial and Trading Terms, Tables of Money, with English and American Values.

illustration of French Coinage, Weights and Measures, Post and Telegraph Rates, \&c.

FRENCH GRAMMAR SELF-TAUGHT.

Crn 8yo. Blue yxapp r $\mathbf{2}$

Red cioth $\mathbf{3}$ :

By J. LAFFITTE, B.-¿s-L. (Principal French Master City of Loudon Schonl) Containing Alphabet, Accent, I,iuison, \&c., Rules of Grammar exemplified with Mode: Sentences, Anxiliary Verbs, Syroptical Table of the fonr Conjugations, Irregulas

Verbs, Exercises, Alphabetical Vocabulary.

KEY to French Grammar Self-Taught Opn 8xo. Blue wrapper 9d. FHENCR SELF-TAUGHT and GRAMMAR

Crown 8yo.

with KEY. Three books bound in one volume.

RRENCH BY HOME STUDY.

Crn 8xo. Blue grapper 49 Banded together.

Three books, banded together: French Self-Tanght, Grammar, and Key.

FRENCR PRONUNCIATION MADE EASY. Croyn 8xo

BY M. H. HÉBERT.

Fourth Edition. Re-written and enlarged Containing the Vowel Sounds, the Consonants, Accentg and Signs, the Nasal, Divisior of Words into Syllables, Quantity, Rules on Proper Nouns, Reading Erercises wis Interlined Phonetic Pronanciation. Key to Reading Exercises.

Complete Catalogue of Foreign Languages gratis on application.

London:-E. MARLBBOROUGH \& Co., Publishers, 51, 0ld Bailey, E.C. 4. 


\section{Marlborough's}

Self-Taught Serie:

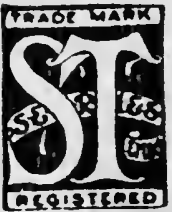

Contains classified Yocabularies and u-eful Phrases and Conver. sations with the BNGLISH PHONETIC PRONUNCIATION n: every word so arranged hat they may be learned $\triangle T$ T GLXNCR.

\section{Prices Net \\ Rod Blue \\ Cloth Wrap.}

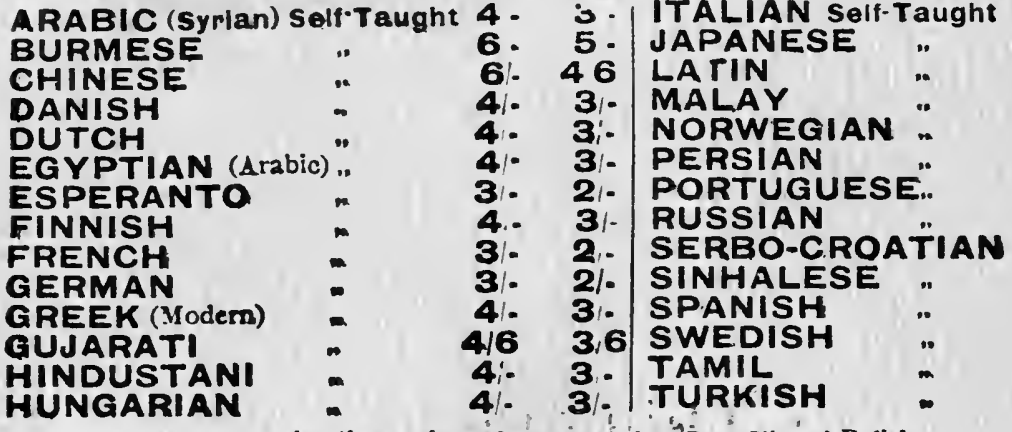

PRICES NI:T Red $\mathrm{Bi}$

Cloth Wrat

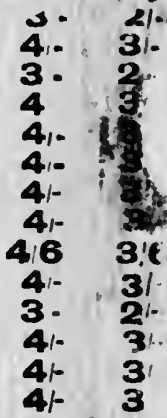

Another volume in preparaion Ben tāli and Polish.

\section{ORAMMARS}

CHINESE GRAMMAR bY JOHN DARROCH. LITT. D.

FRENCH
GERMAN
HINDUSTANI :
ITALIAN
UAPANESE :-
SPANISH
TAMIL

by $J$, LAFFITTE, $B$, es-L.

by W.E. WEBER, M.L -

by S. BLORAYT, M.A. ...

by A. C. PANagulli ...

by H. J. WRINTZ $\quad \ldots \ldots$.

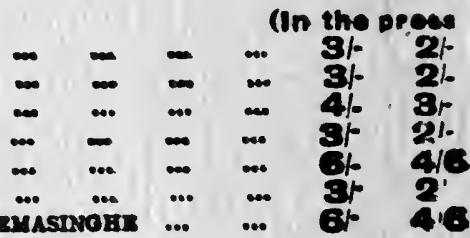

SELF-TAUGHT PHRASE BOOK \& ORAMMAR

(Iniono volumo).

FRENCH self-Taught and Grammap with Koy

\section{GERMAN}

HINDUSTANI:

ITALIAN

JAPANESE

SPANISH

TAMLL
-

HOME STUDY SERIES

(Contains Self.Taught Phrase Book, Grammar and Boy.)

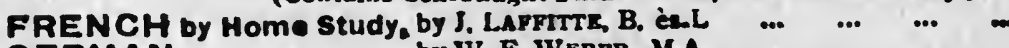

GERMAN

SPANISH

$\infty$

ENGLISH SELF.TAUGHT.

Fop tho FRENCH ... LANGLAIS SANS MAITRE

- GeRMaNis " DER ENGLISCHE DOLMETSCHE

- italians ... Lingleseimparato da se \#

- spaniardos... El inglés para cada cual ..

- Russians ... Cayovqutejs amrjivickato nзutr.

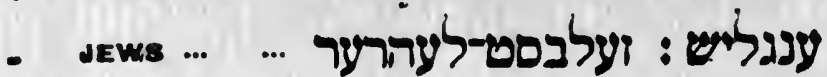

2\%

34. 2,

$8+2$

\&f

8t 2

Lnadon:-R. MAKLBOROUGH \& Co., Pablishers, 51, Old BalloJ, R.C. 
RETURN TO the circulation desk of any

University of California Library

$$
\text { or to the }
$$

NORTHERN REGIONAL LIBRARY FACILITY Bldg. 400, Richmond Field Station University of California Richmond, CA 94804-4698

ALL BOOKS MAY BE RECALLED AFTER 7 DAYS

2-month loans may be renewed by calling

(415) 642-6753

1-year loans may be recharged by bringing books to NRLF

Renewals and recharges may be made 4 days prior to due date

\section{DUE AS STAMPED BELOW}

\section{MAR 151991}

\section{APR 232002}


